\title{
BINARY MICROLENSING EVENTS FROM THE MACHO PROJECT
}

\author{
C. Alcock,${ }^{1,2}$ R. A. Allsman, ${ }^{3}$ D. Alves ${ }^{1,4}$ T. S. Axelrod,${ }^{5}$ D. Baines,${ }^{6}$ A. C. Becker,,${ }^{2,7}$ D. P. Bennett,${ }^{1,2,8}$ \\ A. Bourke, ${ }^{9}$ A. Brakel,${ }^{6}$ K. H. CoOK, ${ }^{1}$ B. Crook,${ }^{6}$ A. Crouch,${ }^{9}$ J. Dan,${ }^{10}$ A. J. Drake, ${ }^{5}$ P. C. Fragile, ${ }^{8}$ \\ K. C. Freeman, ${ }^{5}$ A. Gal-Yam, ${ }^{10}$ M. Geha, ${ }^{1,11}$ J. Gray, ${ }^{9}$ K. Griest, ${ }^{2,12}$ A. Gurtierrez, ${ }^{6}$ A. Heller, ${ }^{10}$ \\ J. Howard,${ }^{6}$ B. R. Johnson, ${ }^{13}$ S. Kaspi,${ }^{10}$ M. Keane,${ }^{14}$ O. Kovo,${ }^{10}$ C. Leach,${ }^{6}$ T. Leach,${ }^{6}$ \\ E. M. Leibowitz, ${ }^{10}$ M. J. LeHNER, ${ }^{15}$ Y. LipKin, ${ }^{10}$ D. MAOZ, ${ }^{10}$ S. L. MARShall, ${ }^{1}$ \\ D. McDowell,${ }^{6}$ S. McKeown, ${ }^{6}$ H. Mendelson, ${ }^{10}$ B. Messenger,${ }^{9}$ D. Minniti,,${ }^{1,16}$ \\ C. Nelson, ${ }^{1,17}$ B. A. Peterson,${ }^{5}$ P. Popowski, ${ }^{1}$ E. Pozza, ${ }^{6}$ P. Purcell, ${ }^{6}$ \\ M. R. Pratt, ${ }^{2,18}$ J. QuinN, ${ }^{8}$ P. J. QuinN, ${ }^{19}$ S. H. RhIE, ${ }^{8}$ A. W. Rodgers, ${ }^{5}$ \\ A. Salmon, ${ }^{6}$ O. Shemmer, ${ }^{10}$ P. Stetson, ${ }^{20}$ C. W. Stubis,${ }^{2,7}$ \\ W. Sutherland, ${ }^{21} \mathrm{~S}$. Thomson, ${ }^{9}$ A. Tomaney, ${ }^{7}$ \\ T. VANDEHEI, ${ }^{2,12}$ A. WALKER, ${ }^{14}$ K. WARD,${ }^{6}$ AND G. WYPER ${ }^{6}$ \\ (THE MACHO/GMAN COLLABORATION) \\ Received 1999 July 26; accepted 2000 March 27
}

\begin{abstract}
We present the light curves of 21 gravitational microlensing events from the first six years of the MACHO Project gravitational microlensing survey that are likely examples of lensing by binary systems. These events were manually selected from a total sample of $\sim 350$ candidate microlensing events that were either detected by the MACHO Alert System or discovered through retrospective analyses of the MACHO database. At least 14 of these 21 events exhibit strong (caustic) features, and four of the events are well fit with lensing by large mass ratio (brown dwarf or planetary) systems, although these fits are not necessarily unique. The total binary event rate is roughly consistent with predictions based upon our knowledge of the properties of binary stars, but a precise comparison cannot be made without a determination of our binary lens event detection efficiency. Toward the Galactic bulge, we find a ratio of caustic crossing to noncaustic crossing binary lensing events of $12: 4$, excluding one event for which we present two fits. This suggests significant incompleteness in our ability to detect and characterize noncaustic crossing binary lensing. The distribution of mass ratios, $N(q)$, for these binary lenses appears relatively flat. We are also able to reliably measure source-face crossing times in four of the bulge caustic crossing events, and recover from them a distribution of lens proper motions, masses, and distances consistent with a population of Galactic bulge lenses at a distance of $7 \pm 1 \mathrm{kpc}$. This analysis yields two systems with companions of $\sim 0.05 M_{\odot}$.
\end{abstract}

Subject headings: binaries: general — dark matter — gravitational lensing stars: low-mass, brown dwarfs

\section{INTRODUCTION}

The search for gravitational microlensing has been very successful since first envisioned by Paczyński (1986). Several teams have reported observations of stellar brightenings that are consistent with the microlensing interpretation (Alcock et al. 1993; Aubourg et al. 1993; Udalski et al. 1993; Alard et al. 1995b). The total number of reported candidate events now exceeds 400 . With second generation surveys

\footnotetext{
${ }^{1}$ Lawrence Livermore National Laboratory, Livermore, CA 94550.

2 Center for Particle Astrophysics, University of California, Berkeley, Berkeley, CA 94720.

${ }^{3}$ Supercomputing Facility, Australian National University, Canberra, ACT 0200, Australia.

${ }^{4}$ Space Telescope Science Institute, 3700 San Martin Drive, Baltimore, MD 21218.

${ }^{5}$ Mt. Stromlo and Siding Spring Observatories, Australian National University, Weston, ACT 2611, Australia.

${ }^{6}$ Reynolds Amateur Photometry Team, Canberra Astronomical Society, Canberra, ACT 0200, Australia.

${ }^{7}$ Departments of Astronomy and Physics, University of Washington, Seattle, WA 98195.

${ }^{8}$ Department of Physics, University of Notre Dame, Notre Dame, IN 46556.

${ }^{9}$ Department of Mathematics and Statistics, Monash University, Clayton, Victoria, 3168, Australia.

${ }^{10}$ School of Physics \& Astronomy and Wise Observatory, Tel-Aviv University, Tel-Aviv 69978, Israel.

${ }^{11}$ Department of Physics, University of California, Davis, Davis, CA 92093.

12 Department of Physics, University of California, San Diego, San Diego, CA 92093.

${ }^{13}$ Tate Laboratory of Physics, University of Minnesota, Minneapolis, MN 55455.

${ }^{14}$ Cerro Tololo Interamerican Observatory, National Optical Astronomy Observatories.

${ }^{15}$ Department of Physics, University of Sheffield, Sheffield s3 7RH, UK.

16 Departmento de Astronomia, P. Universidad Católica, Casilla 104, Santiago 22, Chile.

${ }^{17}$ Department of Physics, University of California, Berkeley, Berkeley, CA 92093.

${ }^{18}$ Center for Space Research, Massachusetts Institute of Technology, Cambridge, MA 02139.

19 European Southern Observatory, Karl-Schwarzschild Strasse 2, D-85748, Garching, Germany.

${ }^{20}$ National Research Council, 5071 West Saanich Road, RR 5, Victoria, BC V8X 4M6, Canada.

${ }^{21}$ Department of Physics, University of Oxford, Oxford OX1 3RH, UK.
} 
now operating, we can expect a similar number of events in the coming years (Udalski, Kubiak, \& Szymański 1997; Palanque-Delabrouille 1997).

By comparing the (efficiency-corrected) observed event rate with model predictions, microlensing surveys have ruled out a large class of dark matter candidates (Alcock et al. 1997b, 1998c; Renault et al. 1997) and appear to have detected a previously unknown component of the GalacticMagellanic Cloud system in lensing objects (Alcock et al. 1997b). The extensive microlensing databases have also allowed important constraints to be placed on Galactic structure (Alcock et al. 1997c, 1998a, 1998b; Paczyński \& Stanek 1998; Zhao, Rich, \& Spergel 1996; Stanek 1996; Stanek et al. 1994).

The gravitational lensing technique uses astronomical sources to backlight a foreground mass distribution. Lensing occurs because of the deflection of light rays by gravity, and most events are well described by the weakfield, small-angle limit of the gravitational scattering of photons. The equation describing the image positions in the lensing plane, for a single point lens is

$$
s=i-R_{\mathrm{E}}^{2} \frac{i-x}{(i-x)^{2}},
$$

where $s, i$, and $\boldsymbol{x}$ are the positions of the source, image, and lens, projected along the line of sight into the lens plane. $R_{\mathrm{E}}$ refers to the Einstein ring radius given by

$$
R_{\mathrm{E}}^{2} \equiv 4 G M\left(\frac{D_{o l} D_{l s}}{D_{o l}+D_{l s}}\right)
$$

where $D_{o l}$ is the distance to the lens, $D_{l s}$ is the lens-source distance, $M$ the mass of the lensing object, and $G$ is the Newtonian gravitational constant. If we define the origin of the lens plane as the position of the lens $(x=0)$, then it is obvious from equation (1) that $i \| s$. This symmetry provides two images during a lensing event. (There is actually a third image of negligible magnification that can be found if we give the lens a finite extent, or if we depart from the weak field approximation.)

The magnification of an image is given by the inverse of the determinant of the lens mapping from image to source (eq. [1]), evaluated at the position of the image. The sum of the two image magnifications, which, if the images are unresolved, is the observed magnification of the source, is

$$
A(t)=\frac{u^{2}+2}{u \sqrt{u^{2}+4}}
$$

where

$$
u(t)=\sqrt{u_{\min }^{2}+\left[2\left(t-t_{0}\right) / \hat{t}\right]^{2}} \text { and } \hat{t}=2 R_{\mathrm{E}} / v_{\perp} .
$$

The lens passes closest to the source at an impact parameter (scaled by $R_{\mathrm{E}}$ ) of $u_{\text {min }}$, at the time $t_{0}$. The variable $\hat{t}$ represents the time it takes the lens to cross its own Einstein diameter (a function of $M$ and $D_{o l} / D_{o s}$ ), given its unknown transverse velocity $v_{\perp}$. This simple form of the event "light curve" is based on point-source and point-lens approximations and an assumption of uniform linear motion between observer, source and lens. As a practical consideration, one must also model additional (unlensed) light that contributes to the source object's baseline brightness and modifies the observed shape of the light curve. This may come from unresolved neighboring stars or possibly from the lens itself. We therefore include a blending parameter for each passband, $f$, which represents the fraction of the photometered object's flux that was lensed.

\section{BINARY MICROLENSING}

Binary lensing is described by the generalization of equation (1) to the case of two deflectors:

$$
s=i-R_{\mathrm{E}}^{2}\left[\epsilon_{1} \frac{i-x_{1}}{\left(i-x_{1}\right)^{2}}+\epsilon_{2} \frac{i-x_{2}}{\left(i-x_{2}\right)^{2}}\right],
$$

where $\epsilon_{1}+\epsilon_{2} \equiv 1$ and $\epsilon_{1}$ and $\epsilon_{2}$ refer to the mass fraction of each lens. $R_{\mathrm{E}}$ now refers to the Einstein ring radius of the complete lens system $\left(M_{1}+M_{2}\right)$.

As with the single lens, equation (4) represents a mapping from the image plane to the source plane. This mapping is well behaved, but the inverse mapping from the source plane to the image plane is multivalued, with each point on the source plane mapping into either three or five points on the image plane. The boundaries between the regions of three and five images in the image plane are referred to as critical curves. These critical curves map into closed caustic curves (or caustics) in the source plane. The shape of a caustic curve is characterized by inwardly curved segments joined at outwardly spiked cusps.

A binary lens light curve can be described by seven parameters if the orbital motion of the lensing objects is neglected. These parameters include the three parameters for a single lens fit $\hat{t}, u_{\min }$, and $t_{0}$, where $u_{\min }$ and $t_{0}$ are now measured with respect to the lensing system's center of mass. The three intrinsic binary parameters are the binary lens separation (scaled by $R_{\mathrm{E}}$ ), $a$, the mass fraction, $\epsilon_{1}$, and the angle between the lens axis and the source trajectory, $\theta$. A final parameter may be added if the source star crosses a caustic: the source radius crossing time $t_{*}$. This represents the time it takes the lens to move relative to the source by an angle equal to the source angular radius - typically 1 hour to 1 day. This may be constrained by observing the caustic crossing duration $2 t_{*} / \sin \phi$, where $\phi$ is the angle between the relative motion vector and the caustic line. An additional blending parameter $f$ must also be included for each passband. An excellent treatment of binary microlensing can be found in Dominik (1998a).

When a source crosses near or over a caustic, the event light curve can differ quite significantly from the standard microlensing light curve. During a caustic crossing, the determinant of the lens mapping in equation (4) goes to zero. This divergence of the magnification factor implies that a point source located on a caustic curve becomes infinitely bright. In this situation, two of the images either merge and disappear or two images are created such that the number of images is greater within the region bounded by the caustic surface. For a realistic source brightness profile, the observed magnification is the weighted mean of the magnification factor over the source, which always leads to finite values.

If one is able to observe a caustic crossing, the temporal width of the transit provides a measurement of $t_{*}$ if $\phi$ is sufficiently constrained by the global microlensing fit. An estimate of the true angular source size then yields the proper motion of the lensing system with respect to the source star. This provides an especially important constraint in binary lensing events seen toward the Magellanic 
Clouds, where there is a distinct difference between the expected relative proper motion of a binary lens in the Galactic dark halo $\left(\mu \sim 30 \mathrm{~km} \mathrm{~s}^{-1} \mathrm{kpc}^{-1}\right)$ and that of a binary lens in the Magellanic Clouds, where the relative proper motion is a function of the velocity dispersion in the cloud $\left(\mu \sim 1 \mathrm{~km} \mathrm{~s}^{-1} \mathrm{kpc}^{-1}\right)$. In general, once a caustic crossing event is detected (the first caustic crossing is unlikely to be resolved because of its short duration but may be inferred from the enhanced magnification between caustics), one is guaranteed a second caustic crossing and, with dense enough sampling through the crossing, an estimate of the lens proper motion.

\subsection{Estimates of the Binary Lensing Rate}

The phenomenology of lensing by two point masses is well known (e.g., Schneider \& Weiss 1986), and the light curve morphology expected in the Galactic microlensing limit well established (e.g., Mao \& Paczyński 1991; Mao \& Di Stefano 1995; Di Stefano \& Perna 1997). Mao \& Paczyński (1991) predicted that $\sim 7 \%$ of all events seen toward the Galactic bulge should show strong evidence of lens binarity and that as many as $\sim 3 \%$ of bulge events might show evidence of a high mass ratio (planetary) binary system, depending upon the abundance of giant planets. The prospect of detecting extrasolar planets through lens binarity has led to much investigation (Gould \& Loeb 1992; Bolatto \& Falco 1994; Bennett \& Rhie 1996; Peale 1997; Gaudi, Naber, \& Sackett 1998a) and distinct methods and detection rates for multiple classes of planetary lensing (Griest \& Safizadeh 1998; Di Stefano \& Scalzo 1999a, 1999b).

The question of the fractional binary lensing rate has recently been analyzed in more detail by Di Stefano (1999), who argues that, in the extreme case in which all lenses are binaries, $\sim 6 \%$ of all events should exhibit caustic crossings. This fraction will obviously decrease if some lenses are single. Thus the majority of binary lensing events should result in multiply peaked light curves or low-amplitude deviations from single-lens light curves without caustic features. However, Di Stefano (1999) appears to underestimate the observational bias toward low impact parameter (small $\left.u_{\min }\right)$ events. Di Stefano (1999) considers all events with $u_{\min } \leq 1$ to be detectable if they are sampled frequently, but in fact the MACHO analysis and Alert System trigger both explicitly exclude lensing events with $u_{\min } \gtrsim 0.75$. Additional cuts on the signal to noise of the stellar brightening also discriminate against the large $u_{\min }$ events, which may lie within the relatively large error bars $(\sim 7 \%)$ of survey system photometry. Furthermore, most of the events seen toward both the Galactic bulge and the Magellanic Clouds are significantly blended, such that the true $u_{\min }$ value is generally smaller than the observed value used for event selection. Figure 2 of Di Stefano (1999) indicates that the fraction of caustic crossing events would be substantially higher if a smaller $u_{\min }$ cut (i.e., $u_{\min } \lesssim 0.3$ ) were used to better reflect the survey team's event selection criteria.

The current microlensing follow-up systems, including the Global Microlensing Alert Network (GMAN; Alcock et al. 1997d), Microlensing Planet Search (MPS) team (Rhie et al. 1999), and Probing Lensing Anomalies Network (PLANET; Albrow et al. 1998a), avoid many of these difficulties with observing strategies optimized to detect shorttimescale, low-level deviations in ongoing events, with photometry at the $\sim 1 \%-2 \%$ level.

\subsection{Previous Binary Lens Observations}

The first unambiguous case of binary microlensing was reported by the OGLE collaboration (OGLE-7; Udalski et al. 1994) and later corroborated with data from the MACHO database (Bennett et al. 1995; Mao et al. 1994). Subsequent binary lens event publications include DUO 2 (Alard, Mao, \& Guibert 1995a), MACHO 95-BLG-12 (Pratt et al. 1996; Albrow et al. 1996), MACHO LMC-1 (Rhie \& Bennett 1996; Dominik \& Hirshfeld 1996), MACHO LMC-9 (Bennett et al. 1996b), MACHO 97-BLG28 (Albrow et al. 1998b), and MACHO 98-SMC-1 (Alcock et al. 1999a; Afonso et al. 2000). In addition, there have been a number of binary lensing events discovered in progress and announced on the web sites of the various microlensing survey and follow-up programs. ${ }^{22}$

\section{OBSERVATIONS}

The purpose of this paper is to present instances of binary lens type light curves that we have detected in our gravitational microlensing survey, using the 50 inch $(1.27 \mathrm{~m})$ telescope at the Mount Stromlo Observatory (Alcock et al. 1997b; Hart et al. 1996; Marshall et al. 1994). These include events toward the Large Magellanic Cloud (LMC), Small Magellanic Cloud (SMC), and Galactic bulge. We have not made a complete assessment of our binary lens detection efficiency, and the events presented here were selected by a variety of different mechanisms. Most were originally discovered with the MACHO Alert System and were subsequently observed to deviate from the standard microlensing light curve. Other events were discovered via our normal analysis procedure (see Alcock et al. 1997b), difference imaging analysis (DIA) on a subset of our database (Alcock et al. 1999b), or during testing of the Alert System software.

Many of the alert events, and most of the unusual events, from 1995 to 1998 were also followed with coordinated observations of the Global Microlensing Alert Network (GMAN; Pratt et al. 1996):

1. These include nightly observations on the Cerro Tololo Inter-American Observatory (CTIO) ${ }^{23} 0.9 \mathrm{~m}$ telescope at Cerro Tololo, Chile, with extremely flexible scheduling allowed by the staff to assist in characterizing the binary events presented here. The observing strategy was optimized in 1997 to target LMC/SMC events at highest priority (and paid off with the real-time detection of binary event MACHO 98-SMC-1; Becker et al. 1998), and thus the frequency of observations of our bulge events decreases.

2. We have also coordinated observations on the Wise Observatory (WISE) $1.0 \mathrm{~m}$ telescope at Mitzpe Ramon, Israel. These observations are generally requested in realtime for a subset of the bulge alert events that show unusual features.

3. For the observing seasons $1995-1996$, we were allotted approximately $50 \%$ of the bulge time at the University of Toronto Southern Observatory (UTSO) $0.6 \mathrm{~m}$ telescope at

${ }^{22}$ EROS: $\quad$ http://www-dapnia.cea.fr/Spp/Experiences/EROS/ alertes.html; MACHO: http://darkstar.astro.washington.edu; MPS: http://bustard.phys.nd.edu/MPS/; OGLE: http://www.astrouw.edu.pl/ftp/ ogle/ogle2/ews/ews.html; PLANET: http://www.astro.rug.nl/planet/ index.html.

${ }^{23}$ Cerro Tololo Inter-American Observatory, National Optical Astronomy Observatories, operated by the Association of Universities for Research in Astronomy, Inc., under cooperative agreement with the National Science Foundation. 
Las Campanas, Chile, for microlensing follow-up. Several events were followed at high frequency, and three of these appear here as binary candidates.

4. Starting in 1997, we arranged microlensing follow-up observations at the Mount Stromlo Observatory 30 inch (76 cm; MSO30) telescope in Canberra, Australia, with the Reynolds Amateur Photometry Team (RAPT). This group of amateur astronomers staffs the telescope nightly, on a volunteer basis.

5. The Microlensing Planet Search (MPS) team began its pilot season of observations in 1997 and has obtained use of the MSO 74 inch (1.88 m; MSO74) telescope for rapid, precise monitoring of ongoing Galactic bulge events.

The MACHO survey data from the Mt. Stromlo 50 inch telescope are generally reduced within hours after acquisition using the SoDOPHOT photometry routine (Bennett et al. 1993), which was developed from DOPHOT (Schechter, Mateo, \& Saha 1993). The error bars presented here are the standard SoDOPHOT (or DOPHOT) error estimates with a $1.4 \%$ uncertainty added in quadrature. As the 50 inch data are reduced, the photometry is sent through the MACHO Alert filter for the purpose of detecting microlensing events in progress. Thirteen of the 21 events presented here were first detected with the MACHO Alert System, although two of these events were not found while in progress.

All CTIO, MSO30, and UTSO data are reduced in real time using a suite of scripts, written in PERL, which make use of the DAOPHOT package (Stetson 1987). After the event has returned to baseline, CTIO, MSO30, UTSO, and WISE data are reduced en masse using the ALLFRAME package (Stetson 1994). To assemble each light curve, the target star's brightness is finally normalized with respect to several hundred neighboring stars. The resulting photometric error bars are multiplied by a factor of 1.5 to account for additional scatter in the time series not accounted for by the formal ALLFRAME error estimates, such as flat fielding and normalization errors. The MSO74 data are reduced and normalized using a slightly modified version of SoDOPHOT.

Finally, we would like to emphasize that for some of these fits there are probably degeneracies between model parameters. That is, the fit presented is not unique but does represent a local minimum in parameter space. Is it likely that for some of these events, particularly the poorly sampled ones, there are different lens configurations whose light curves, as sampled by our observations, are similar to the fits presented here (see Dominik 1999). There are, however, systematic approaches to determining all physical solutions for a set of microlensing data (e.g., Di Stefano \& Perna 1997; Albrow et al. 1999b, 1999c).

\section{BINARY LENSING EVENTS}

Characteristics of the MACHO objects that received the lensed flux are presented in Table 1. Each object is given an event name in addition to the MACHO star identification number that has been previously assigned. For each object, we include the transformation to Cousins $V$ and $V-R$, calibrated using Alcock et al. (1999c). From the blending parameters included in the binary microlensing fit, we can also estimate the true brightness and color of the star that was lensed. This information is also included in Table 1. For all events, we will refer to the (sometimes blended) object identified in the MACHO database as the "object" and to the actual star in the blend that was lensed as the "source." Fit parameters for these binary microlensing events are given in Tables 2 and 3. For each event, we present a light curve of these fits, as well as a schematic of the critical and caustic curves induced by the binary lens (Figs. 1-44). The data used in the fits may be found on the World Wide

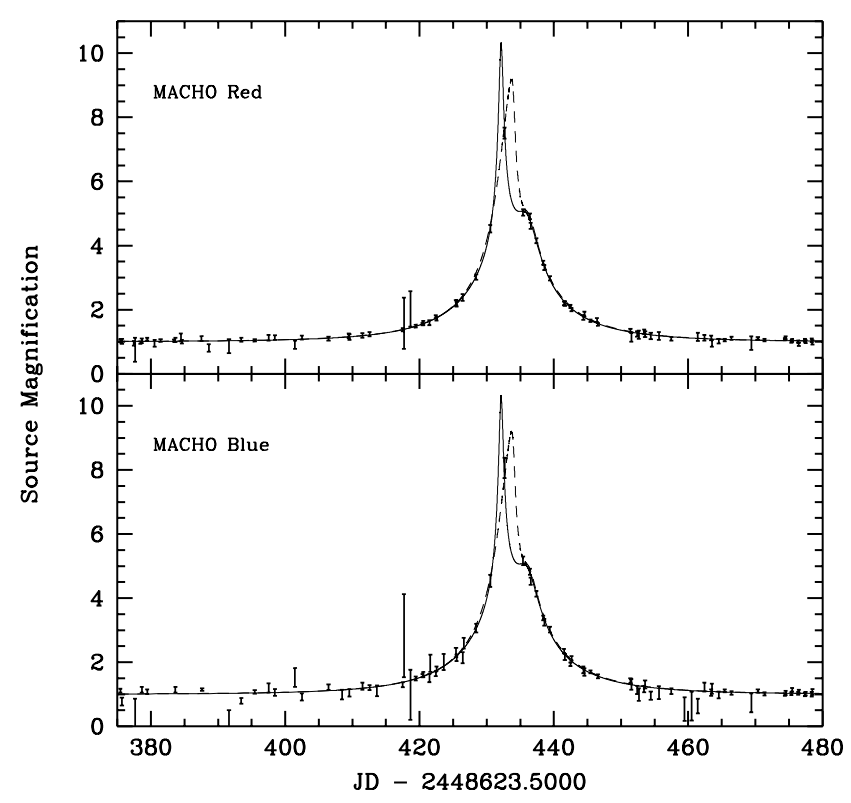

FIG. 1.- Light curve of MACHO event LMC-1, including fits indicating a "planetary" mass secondary lens (dashed line) and a more standard binary system (solid line).

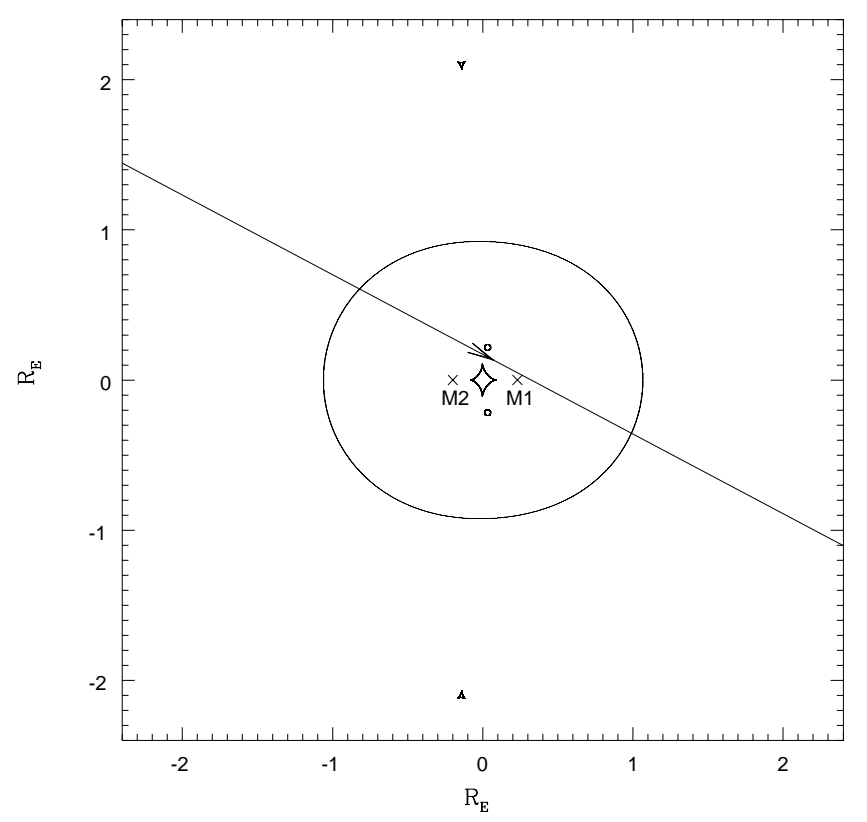

FIG. 2.-Location of the (cuspy) caustic and (smooth) critical curves for the LMC-1 standard binary lens fit (solid line) presented in Fig. 1. The coordinate system, whose origin is at the center of mass, indicates distance in units of the system's Einstein ring radius $R_{\mathrm{E}}$. Also shown are the locations of the lensing objects, and the trajectory of the source through the caustic structure. 
TABLE 1

CANDidate Lensed Source Stars

\begin{tabular}{|c|c|c|c|c|c|c|c|}
\hline \multirow[b]{2}{*}{ EVENT } & \multirow[b]{2}{*}{ MACHO } & \multirow[b]{2}{*}{ R.A. (J2000) } & \multirow[b]{2}{*}{ decl. (J2000) } & \multicolumn{2}{|c|}{ МАСНО ОВЈеСТ ${ }^{\mathrm{a}}$} & \multicolumn{2}{|c|}{ Lensed Source Star ${ }^{b}$} \\
\hline & & & & $V$ & $V-R$ & $V$ & $V-R$ \\
\hline LMC-1 (solid line in figures) ...... & 79.5628 .1547 & 051444.5 & -684800.1 & 19.73 & 0.54 & 19.75 & 0.55 \\
\hline LMC-1 (dashed line) ............... & & $\ldots$ & $\ldots$ & 19.73 & 0.54 & 19.78 & 0.55 \\
\hline LMC-9........................... & 80.6468 .2746 & 052020.2 & -691511.8 & 19.62 & 0.28 & 21.43 & 0.55 \\
\hline LMC-10 ........................ & 18.3324 .1765 & 050116.0 & -690733.1 & 19.49 & 0.13 & 21.57 & 0.13 \\
\hline 98-SMC-1 ....................... & 208.15683 .4237 & 004535.2 & -725234.1 & 21.62 & 0.20 & 22.28 & 0.09 \\
\hline OGLE-7/119-A .................. & 119.20226 .2119 & 180335.7 & -294201.2 & 19.16 & 0.84 & 20.21 & 0.90 \\
\hline $403-\mathrm{C} \ldots \ldots \ldots \ldots \ldots \ldots \ldots$ & 403.47793 .2961 & 175557.9 & -292612.1 & 18.87 & 1.45 & 19.62 & 1.41 \\
\hline 94-BLG-4 ........................ & 118.18141 .731 & 175836.7 & -300219.2 & 17.92 & 1.06 & 17.87 & 1.07 \\
\hline 95-BLG-12 „.................... & 120.21263 .1213 & 180604.7 & -295238.1 & 18.88 & 0.92 & 20.39 & 0.81 \\
\hline 96-BLG-3... & 119.19444.2055 & 180145.5 & -294946.8 & 20.00 & 0.78 & 21.48 & 0.73 \\
\hline 96-BLG-4 .... & 105.21417.101 & 180611.9 & -281652.7 & 16.25 & 1.07 & 16.21 & 1.06 \\
\hline 97-BLG-1 .............. & 113.18674 .756 & 175953.3 & -290907.8 & 17.41 & 0.95 & 17.46 & 0.92 \\
\hline 97-BLG-24 (solid line) ... & 101.20650.1216 & 180420.2 & -272445.2 & 18.01 & 0.67 & 19.96 & 0.65 \\
\hline 97-BLG-24 (dashed line) ... & $\ldots$ & $\ldots$ & $\ldots$ & 18.01 & 0.67 & 20.7 & 0.64 \\
\hline 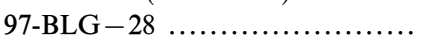 & 108.18951 .593 & 180033.7 & -280110.4 & 18.10 & 1.18 & 18.19 & 1.25 \\
\hline 97-BLG-41 ...................... & 402.47862 .1576 & 175620.6 & -284741.9 & 19.54 & 1.29 & $\ldots$ & $\ldots$ \\
\hline 98-BLG-12 ...................... & 179.21577.1740 & 180631.7 & -261601.5 & 18.86 & 0.96 & 20.83 & 1.10 \\
\hline 98-BLG-14 (solid line) ............. & 401.48408 .649 & 175908.9 & -282454.6 & 17.70 & 1.20 & 18.42 & 1.19 \\
\hline 98-BLG-14 (dashed line) & $\ldots$ & $\ldots$ & $\ldots$ & 17.70 & 1.20 & 17.61 & 1.19 \\
\hline 98-BLG-16 ...................... & 402.47863 .110 & 175618.1 & -284604.9 & 16.66 & 1.19 & 20.28 & 1.29 \\
\hline 98-BLG-42 „..................... & 101.21045 .2528 & 180512.6 & -270547.1 & 19.33 & 0.85 & 20.20 & 0.86 \\
\hline 97-BLG-d2 & 108.19073 .2291 & 180039.5 & -283443.8 & $\ldots$ & $\ldots$ & 20.14 & 0.87 \\
\hline 108-E ....... & 108.19333 .1878 & 180121.1 & -283239.4 & 17.41 & 1.04 & 17.52 & 1.21 \\
\hline 176-A ...... & 176.19219.978 & 180104.4 & -273041.3 & 18.74 & 1.27 & 19.11 & 1.30 \\
\hline
\end{tabular}

NoTES.-Units of right ascension are hours, minutes, and seconds, and units of declination are degrees, arcminutes, and arcseconds. Information on the lensed MACHO objects and deblended source stars. Events LMC-1, 97-BLG-24, and 98-BLG-14 are each presented with two fits of similar significance but different event parameters. We do not present a binary microlensing fit for event 97-BLG-41, and we include only an estimate of the MACHO object's baseline flux. Event 97-BLG-d2 was found through difference image analysis (DIA), which uniquely identifies the lensed source.

a Standard magnitudes and color of the MACHO object that received the lensed flux.

b Standard magnitudes and color of the actual lensed source star, as determined from the blend fraction in the binary lens fit.

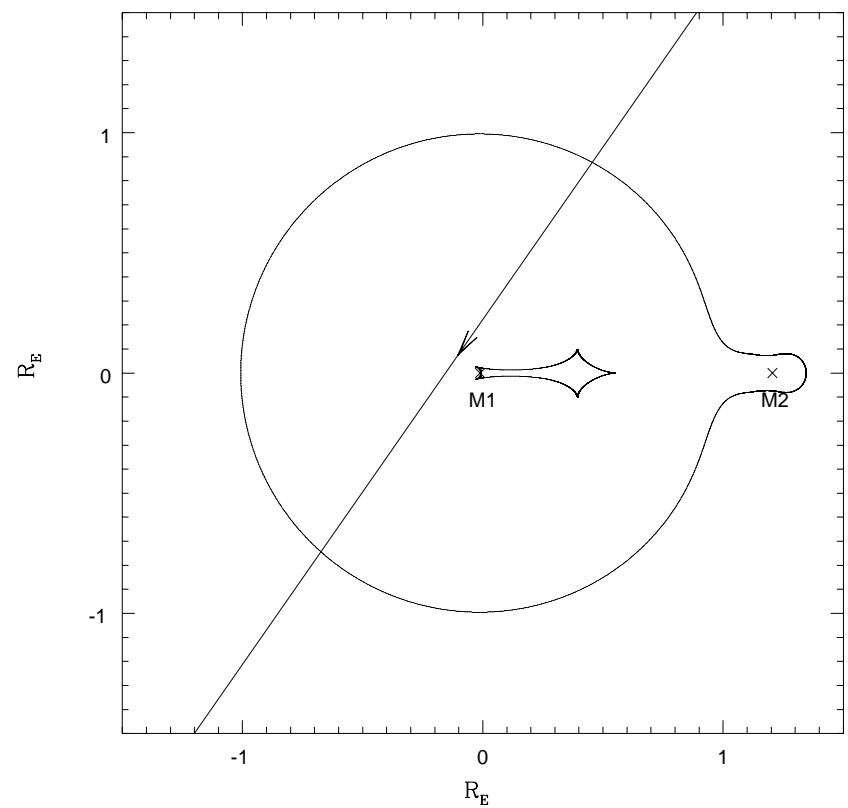

FIG. 3.-Location of the (cuspy) caustic and (smooth) critical curves for the LMC-1 planetary binary lens fit (dashed line) presented in Fig. 1. The coordinate system, whose origin is at the center of mass, indicates distance in units of the system's Einstein ring radius $R_{\mathrm{E}}$. Also shown are the locations of the lensing objects, and the trajectory of the source through the caustic structure.

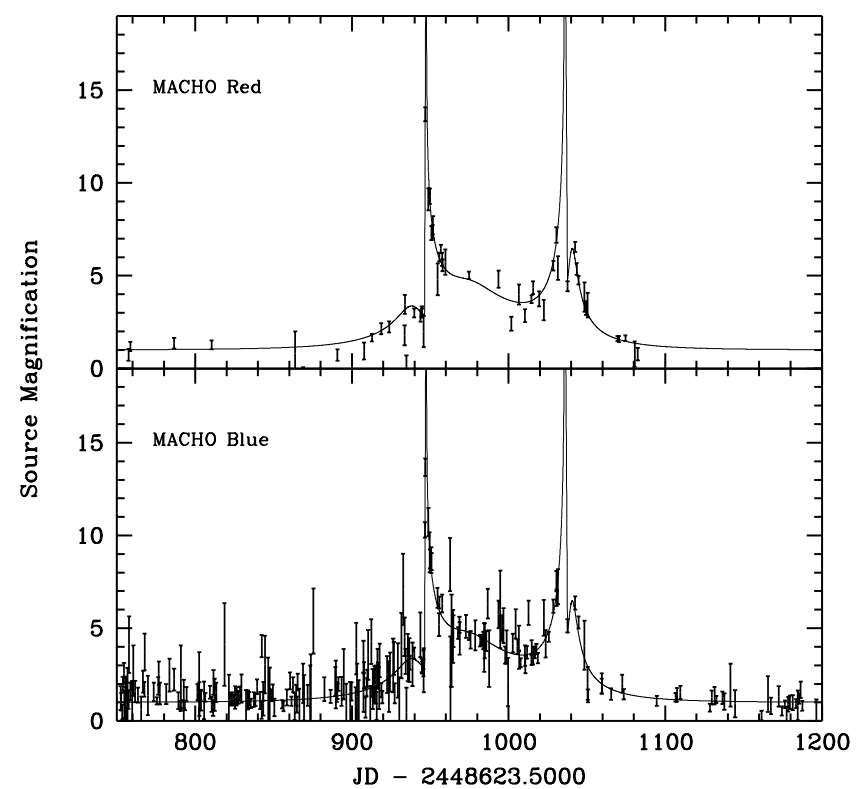

FIG. 4. - Light curve of MACHO event LMC-9, including our fit to binary microlensing. 
TABLE 2

Binary Microlensing Event Parameters

\begin{tabular}{|c|c|c|c|c|c|c|c|c|}
\hline Event & $\chi^{2} /$ d.o.f. & $\hat{t}$ & $t_{0}{ }^{\mathrm{a}}$ & $u_{\min }$ & $a$ & $\begin{array}{c}\theta \\
(\mathrm{rad})\end{array}$ & $M_{1} / M_{2}$ & $\begin{array}{c}t_{*} \\
\text { (days) }\end{array}$ \\
\hline LMC-1 (solid line in figures)...... & 2794.9/2179 & 35.54 & 433.58 & 0.150 & -0.430 & -0.488 & 0.861 & 0.112 \\
\hline LMC-1 (dashed line)... & $2799.4 / 2179$ & 34.55 & 433.58 & -0.127 & 1.217 & 4.104 & 108.890 & 0.122 \\
\hline LMC-9 ......................... & $1476.5 / 848$ & 143.12 & 979.59 & -0.054 & 1.657 & 0.086 & 1.627 & 0.651 \\
\hline LMC-10 ........................ & $1672.9 / 1240$ & 151.05 & 585.74 & 0.102 & 0.823 & -3.375 & 0.034 & $\ldots$ \\
\hline 98-SMC-1 ..................... & $1771.9 / 1583$ & 147.58 & 2354.93 & 0.046 & 0.664 & -0.180 & 0.388 & 0.116 \\
\hline OGLE-7/119-A ................. & 1997.6/1407 & 169.04 & 550.04 & 0.077 & 1.045 & -0.940 & 1.212 & 0.212 \\
\hline 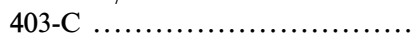 & $318.7 / 342$ & 21.37 & 1688.75 & -0.089 & 1.227 & 3.082 & 0.556 & $\ldots$ \\
\hline 94-BLG-4 ..................... & $923.5 / 1418$ & 10.66 & 884.13 & 0.028 & -1.068 & 0.302 & 18.084 & 0.096 \\
\hline 95-BLG-12 .................... & $1413.4 / 1466$ & 307.41 & 1264.07 & 0.106 & 0.421 & -0.664 & 2.148 & 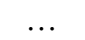 \\
\hline 96-BLG-3... & $1934.8 / 1542$ & 182.45 & 1545.66 & 0.043 & -0.436 & 3.530 & 0.392 & 0.086 \\
\hline $96-\mathrm{BLG}-4 \ldots$ & $1286.0 / 1605$ & 162.44 & 1815.29 & 0.378 & 7.454 & -0.011 & 1.140 & $\ldots$ \\
\hline 97-BLG-1 .... & $1237.7 / 1305$ & 68.52 & 1884.02 & 0.234 & 0.931 & 2.583 & 0.418 & 0.527 \\
\hline 97-BLG-24 (solid line) ............. & $1230.6 / 943$ & 30.77 & 1968.38 & 0.319 & 2.077 & -3.967 & 4.236 & 0.050 \\
\hline 97-BLG-24 (dashed line) .......... & $1235.0 / 943$ & 45.49 & 1970.82 & 0.0003 & 1.752 & 0.988 & 0.035 & 0.197 \\
\hline 97-BLG-28 „.................... & 2734.9/1404 & 52.76 & 1991.87 & 0.225 & 0.707 & 1.437 & 0.210 & 0.760 \\
\hline 98-BLG-12 …............... & $440.5 / 507$ & 239.92 & 2321.95 & 0.163 & 0.793 & 11.346 & 0.681 & $\ldots$ \\
\hline 98-BLG-14 (solid line) ............ & $809.4 / 714$ & 107.65 & 2322.32 & 0.214 & 0.541 & 3.531 & 4.504 & $\ldots$ \\
\hline 98-BLG-14 (dashed line) ......... & $814.4 / 714$ & 74.05 & 2323.56 & 0.396 & 1.213 & 4.429 & 0.0857 & $\ldots$ \\
\hline 98-BLG-16 ..................... & $461.2 / 616$ & 69.50 & 2316.57 & 0.140 & 0.758 & 3.699 & 1.476 & 0.163 \\
\hline 98-BLG-42 …................ & $890.4 / 395$ & 49.19 & 2422.41 & -0.028 & 1.260 & 3.328 & 3.065 & 0.109 \\
\hline 97-BLG-d2 ..................... & $314.1 / 584$ & 92.65 & 1971.07 & -0.326 & 0.965 & 1.766 & 0.390 & . \\
\hline 108-E....... & $934.4 / 597$ & 71.30 & 1927.66 & 0.747 & 0.637 & -2.609 & 1.514 & 0.557 \\
\hline $176-\mathrm{A} \ldots$ & $684.0 / 578$ & 60.02 & 1230.06 & 0.108 & 0.965 & 0.204 & 1.514 & 0.068 \\
\hline
\end{tabular}

NoteS.-List of binary microlensing event parameters, as described in $\S 2$, for each of our candidates. Also included are the binary microlensing fit $\chi^{2}$ and the degrees of freedom (equal to the number of data points minus 1 for each constraint listed here, and minus 2 for each passband with observations in Table 3). Events LMC-1, 97-BLG-24, and 98-BLG-14 are each presented with two fits of similar significance but different event parameters.

a (JD-2448623.50).

TABLE 3

Binary Microlensing Event Blend Parameters

\begin{tabular}{|c|c|c|c|c|c|c|c|c|c|}
\hline Event & $f_{\text {MACHO }_{R}}$ & $f_{\mathrm{MACHO}_{B}}$ & $f_{\mathrm{CTIO}_{R}}$ & $f_{\mathrm{CTIO}_{B}}$ & $f_{\mathrm{MSO} 0_{R}}$ & $f_{\mathrm{MSO} 74_{R}}$ & $f_{\mathrm{UTSO}_{R}}$ & $f_{\text {UTSO }_{V}}$ & $f_{\mathrm{wISE}_{R}}$ \\
\hline LMC-1 (solid line in figures)....... & 0.99 & 0.98 & $\ldots$ & $\ldots$ & $\ldots$ & $\ldots$ & $\cdots$ & $\cdots$ & $\ldots$ \\
\hline LMC-1 (dashed line) ................ & 0.96 & 0.95 & $\ldots$ & $\ldots$ & $\ldots$ & $\ldots$ & $\ldots$ & .. & $\ldots$ \\
\hline LMC-9 .......................... & 0.26 & 0.17 & ... & $\ldots$ & $\ldots$ & $\ldots$ & $\ldots$ & .. & $\ldots$ \\
\hline LMC-10 ........................ & 0.15 & 0.15 & $\ldots$ & $\ldots$ & $\ldots$ & $\ldots$ & $\ldots$ & ... & $\ldots$ \\
\hline 98-SMC-1 …................ & 0.47 & 0.56 & 0.79 & 1.07 & $\ldots$ & $\ldots$ & $\ldots$ & $\ldots$ & $\ldots$ \\
\hline OGLE-7/119-A ................. & 0.41 & 0.37 & $\ldots$ & $\ldots$ & $\ldots$ & $\ldots$ & $\ldots$ & $\ldots$ & $\ldots$ \\
\hline 403-C ......................... & 0.48 & 0.50 & $\ldots$ & $\ldots$ & $\ldots$ & $\ldots$ & $\ldots$ & $\ldots$ & $\ldots$ \\
\hline 94-BLG-4 ...................... & 1.06 & 1.04 & $\ldots$ & $\ldots$ & $\ldots$ & $\ldots$ & $\ldots$ & $\ldots$ & $\ldots$ \\
\hline 95-BLG-12 ................... & 0.22 & 0.26 & 0.26 & $\ldots$ & $\ldots$ & $\ldots$ & 0.27 & 0.26 & 0.31 \\
\hline 96-BLG-3 $\ldots \ldots \ldots \ldots \ldots \ldots \ldots \ldots \ldots$ & 0.23 & 0.25 & 0.35 & $\ldots$ & $\ldots$ & $\ldots$ & 0.33 & $\ldots$ & $\ldots$ \\
\hline 96-BLG-4 ..................... & 1.03 & 1.03 & 1.05 & $\ldots$ & $\ldots$ & $\ldots$ & 1.01 & $\ldots$ & $\ldots$ \\
\hline 97-BLG-1 ..................... & 0.93 & 0.97 & 0.87 & $\ldots$ & $\ldots$ & $\ldots$ & $\ldots$ & $\ldots$ & $\ldots$ \\
\hline 97-BLG-24 (solid line) ............. & 0.16 & 0.17 & $\ldots$ & $\ldots$ & 0.15 & $\ldots$ & $\ldots$ & $\ldots$ & 0.21 \\
\hline 97-BLG-24 (dashed line) .......... & 0.08 & 0.08 & $\ldots$ & $\ldots$ & 0.08 & $\ldots$ & $\ldots$ & $\ldots$ & 0.11 \\
\hline 97-BLG-28 ................... & 1.00 & 0.90 & 0.97 & $\ldots$ & $\ldots$ & $\ldots$ & $\ldots$ & $\ldots$ & $\ldots$ \\
\hline 98-BLG-12 „................... & 0.17 & 0.14 & 0.17 & $\ldots$ & $\ldots$ & 0.16 & $\ldots$ & $\ldots$ & $\ldots$ \\
\hline 98-BLG-14 (solid line) ............ & 0.51 & 0.52 & 0.52 & $\ldots$ & $\ldots$ & 0.48 & $\ldots$ & $\ldots$ & $\ldots$ \\
\hline 98-BLG-14 (dashed line) .......... & 1.08 & 1.10 & 1.07 & $\ldots$ & $\ldots$ & 0.99 & $\ldots$ & $\ldots$ & $\ldots$ \\
\hline 98-BLG-16 ..................... & 0.04 & 0.03 & 1.10 & $\ldots$ & $\ldots$ & 1.07 & $\ldots$ & $\ldots$ & 0.04 \\
\hline 98-BLG-42 .................... & 0.46 & 0.45 & $\ldots$ & $\ldots$ & 0.73 & 0.74 & $\ldots$ & $\ldots$ & $\ldots$ \\
\hline 97-BLG-d2 ................... & 0.30 & 0.14 & $\ldots$ & $\ldots$ & $\ldots$ & $\ldots$ & $\ldots$ & $\cdots$ & $\cdots$ \\
\hline 108-E........................ & 1.10 & 0.86 & $\ldots$ & $\cdots$ & $\ldots$ & $\ldots$ & $\ldots$ & $\ldots$ & $\ldots$ \\
\hline 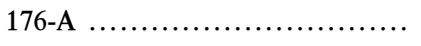 & 0.74 & 0.70 & $\ldots$ & $\ldots$ & $\ldots$ & $\ldots$ & $\ldots$ & $\ldots$ & $\ldots$ \\
\hline
\end{tabular}

NoTES.-List of blending parameters for each of our candidate binary microlensing events. The parameter $f$ represents the fraction of the object's flux that was lensed in each respective passband. Events LMC-1, 97-BLG-24, and 98-BLG-14 are each presented with two fits of similar significance but different event parameters. 


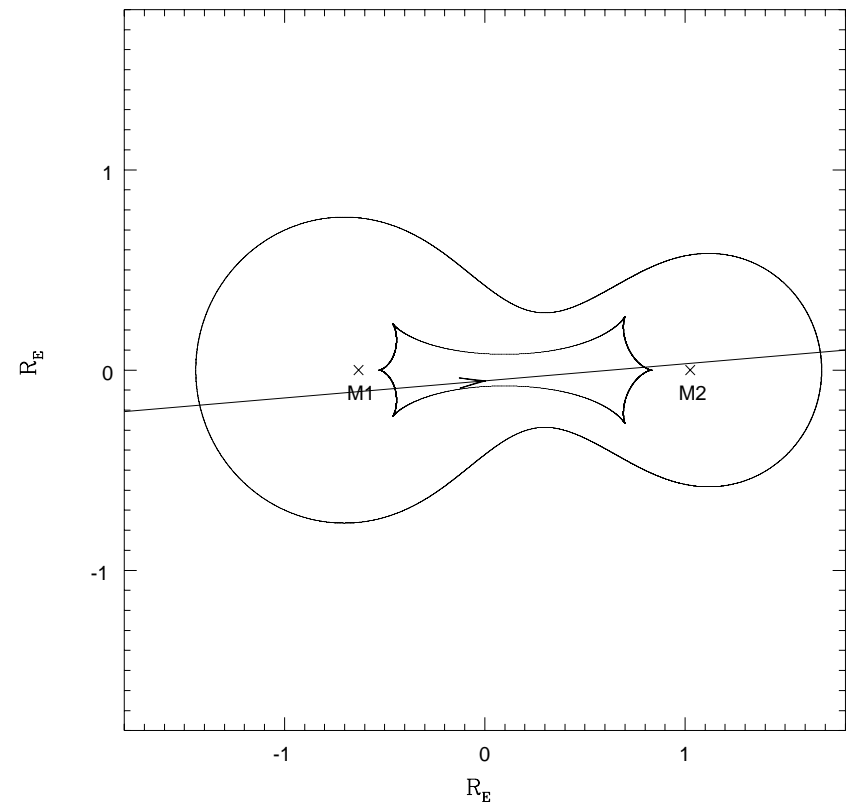

FIG. 5.- Location of the (cuspy) caustic and (smooth) critical curves for the LMC-9 binary lens fit presented in Fig. 4. The coordinate system, whose origin is at the center of mass, indicates distance in units of the system's Einstein Ring radius $R_{\mathrm{E}}$. Also shown are the locations of the lensing objects, and the trajectory of the source through the caustic structure.

Web. ${ }^{24}$ Individuals are encouraged to attempt other binary lens fits to these data.

Figures 45 and 46 display the distribution of $V, V-R$ for $\sim 3,000$ neighboring sources in the same $5^{\prime} \times 5^{\prime}$ " chunk" (1/64 of MACHO's focal plane) as the lensed object, for Magellanic Cloud and Galactic bulge sources, respectively.

${ }^{24}$ The data used in the fits may be found on the World Wide Web at http://wwwmacho.anu.edu.au/ and http://wwwmacho.mcmaster.ca/.

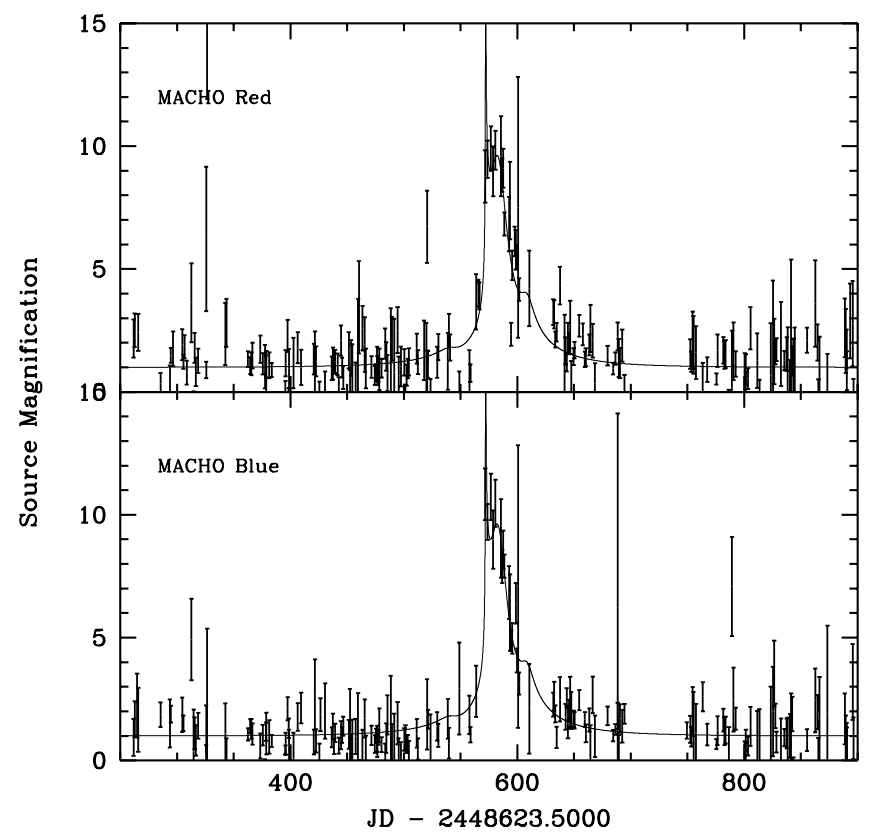

FIG. 6.- - Light curve of MACHO event LMC-10, including our fit to binary microlensing.

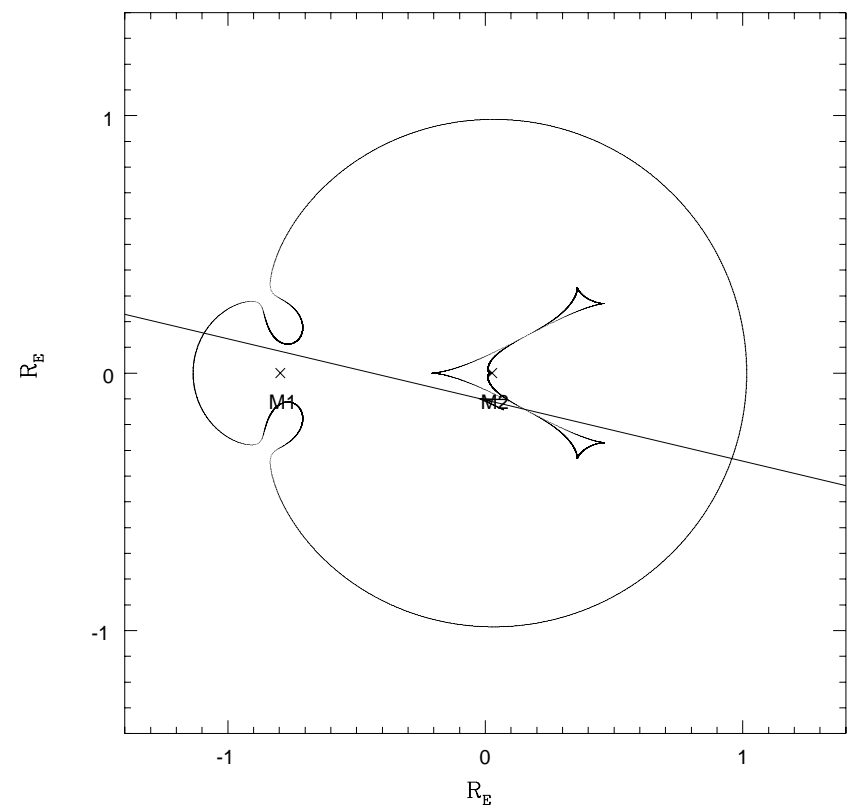

FIG. 7.- Location of the (cuspy) caustic and (smooth) critical curves for the LMC-10 binary lens fit presented in Fig. 6. The coordinate system, whose origin is at the center of mass, indicates distance in units of the system's Einstein Ring radius $R_{\mathrm{E}}$. Also shown are the locations of the lensing objects, and the trajectory of the source through the caustic structure.

The solid circle indicates the location of the MACHO object, and the open circle represents the location of the lensed source, as determined from the blending parameters.

In the following, we discuss each of the 21 events individually.

\section{1. $L M C-1$ (79.5628.1547)}

This event was the first LMC microlensing event reported by the MACHO collaboration (Alcock et al. 1993). The lensed object does not significantly change color during the

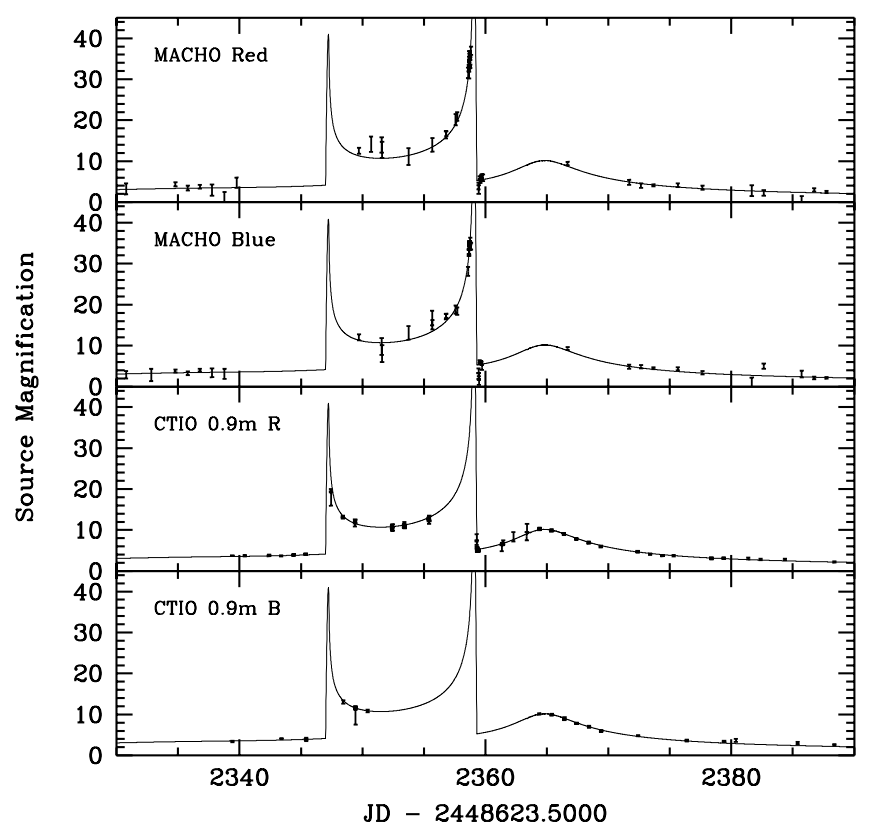

FIG. 8.- - Light curve of MACHO event 98-SMC-1, including our fit to binary microlensing. 


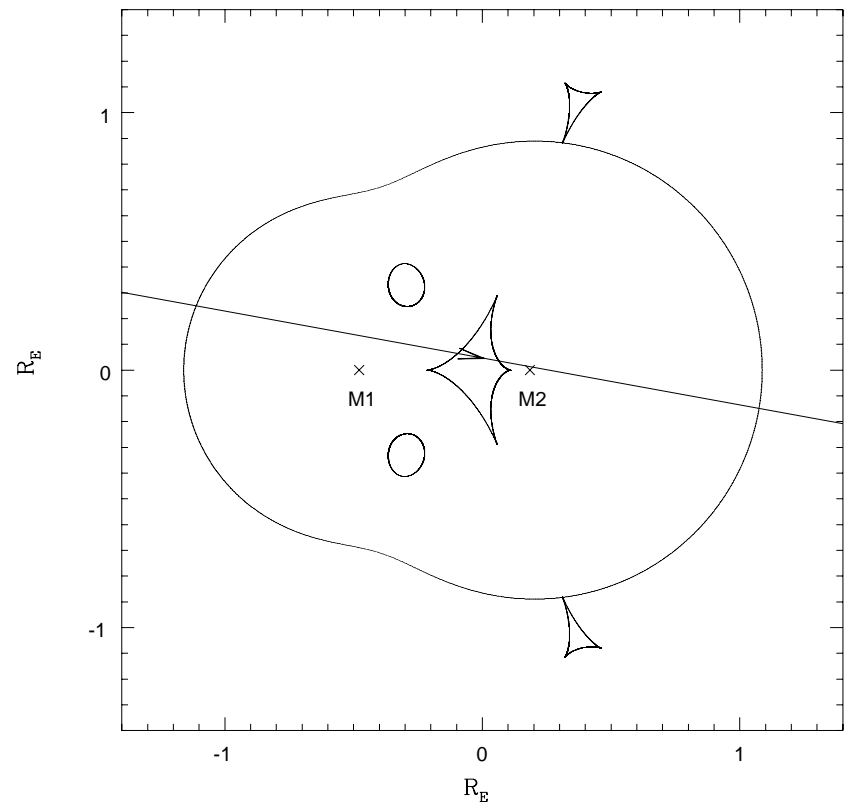

FIG. 9.- - Location of the (cuspy) caustic and (smooth) critical curves for the 98-SMC-1 binary lens fit presented in Fig. 8. The coordinate system, whose origin is at the center of mass, indicates distance in units of the system's Einstein Ring radius $R_{\mathrm{E}}$. Also shown are the locations of the lensing objects, and the trajectory of the source through the caustic structure.

event, and its color and magnitude indicate that it is a slightly reddened red clump giant star in the LMC. We thus expect a priori that a large fraction of the MACHO object's brightness should be lensed. A difference image photometry (DIP; Tomaney \& Crotts 1996) analysis of the event images indicates a very small centroid shift between the photometered object and lensed source, $00^{\prime \prime} 1 \pm 0$ ". 1 , also suggesting the clump giant was lensed.

The light curve is consistent with single lens microlensing, except for a shoulder shortly after the light curve peak. This suggests a short timescale structure that was not resolved at

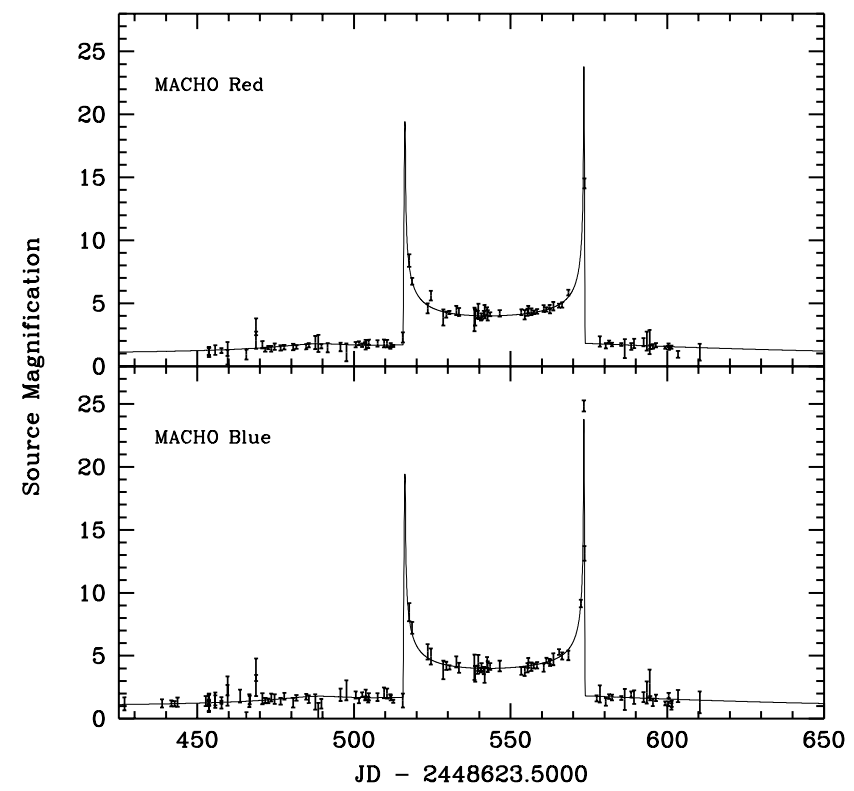

FIG. 10.- Light curve of MACHO event 119-A, including our fit to binary microlensing.

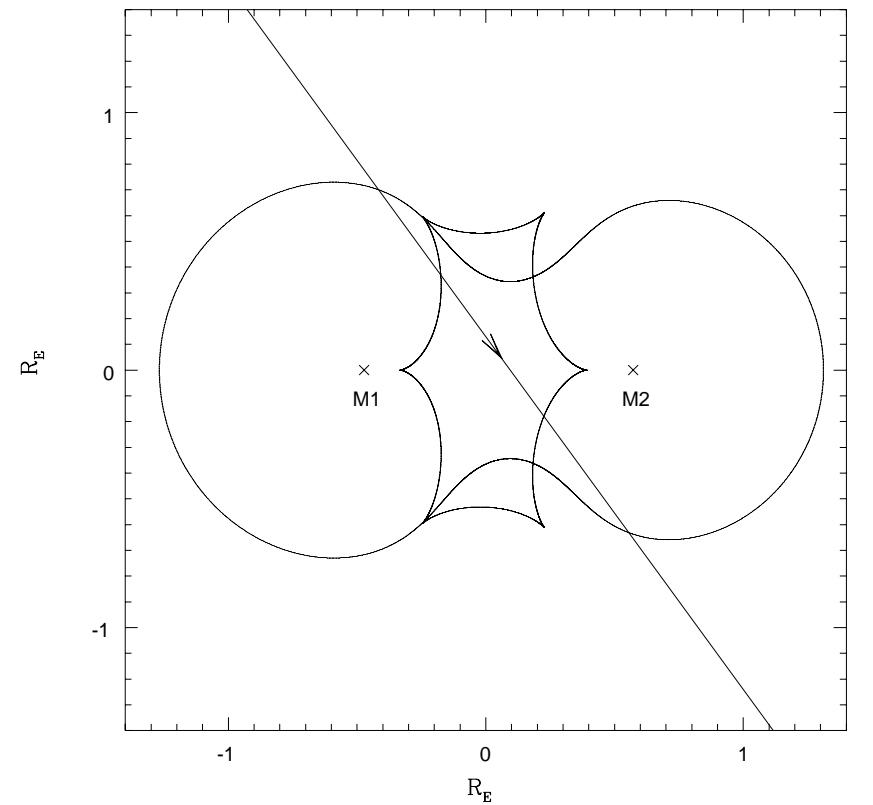

FIG. 11.- - Location of the (cuspy) caustic and (smooth) critical curves for the 119-A binary lens fit presented in Fig. 10. The coordinate system, whose origin is at the center of mass, indicates distance in units of the system's Einstein Ring radius $R_{\mathrm{E}}$. Also shown are the locations of the lensing objects, and the trajectory of the source through the caustic structure.

the sampling of the survey system. Binary fits for this event have been done by Dominik \& Hirshfeld (1996), and the two fits displayed in Figure 1 were previously presented by Rhie \& Bennett (1996). The similarity to standard microlensing implies the fit resides in a class of fits with small lens separations (solid line in Figs. 1 and 2) or large mass ratios (dashed line in Figs. 1 and 3).

The measured $\hat{t} \sim 35$ days for both of these fits yields an expectation value for the mass of the lensing system of $\sim 0.1$ $M_{\odot}$ (Alcock et al. 1993; Dominik 1998b). For the large mass ratio fit, this implies a secondary companion of $\sim 1$

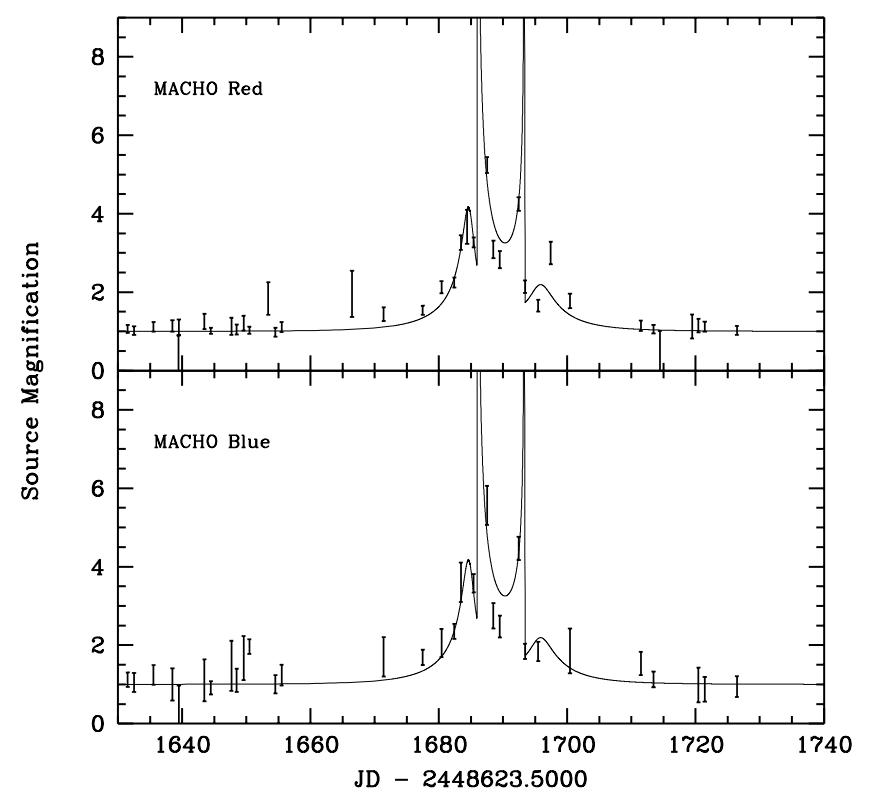

FIG. 12.- Light curve of MACHO event 403-C, including our fit to binary microlensing. 


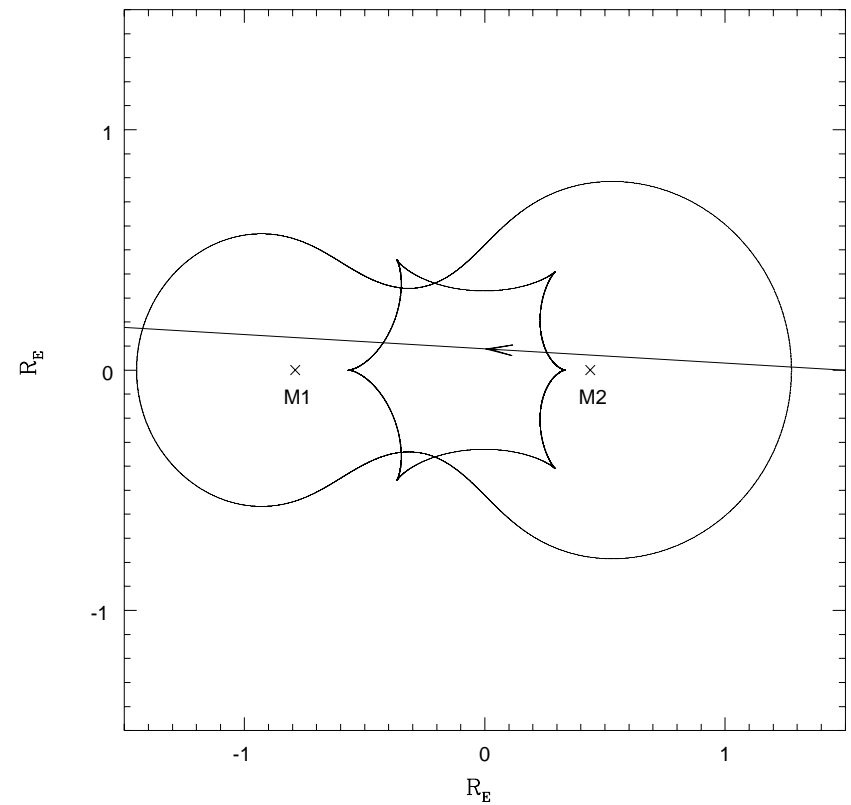

FIG. 13.-Location of the (cuspy) caustic and (smooth) critical curves for the 403-C binary lens fit presented in Fig. 12. The coordinate system, whose origin is at the center of mass, indicates distance in units of the system's Einstein Ring radius $R_{\mathrm{E}}$. Also shown are the locations of the lensing objects, and the trajectory of the source through the caustic structure.

Jupiter mass. However, since there were no follow-up efforts in place at the time of this event, this degeneracy between fits cannot be broken.

\section{2. $L M C-9(80.6468 .2746)$}

LMC-9 is the most anomalous lensing event discovered toward the LMC, and it displays the light curve structure of a typical caustic crossing binary lens event with similar mass lenses (see Fig. 4). Prior analysis of this event can be found in Bennett et al. (1996b). The lensed object appears to be on the red side of the LMC main sequence, at the level of

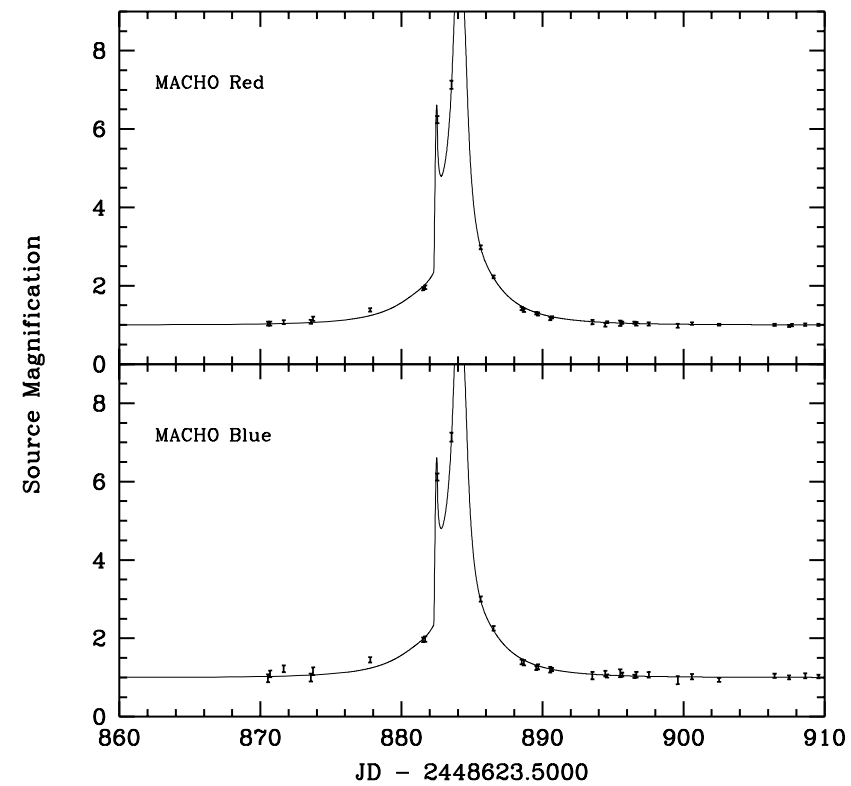

FIG. 14.- - Light curve of MACHO event 94-BLG-4, including our fit to binary microlensing.

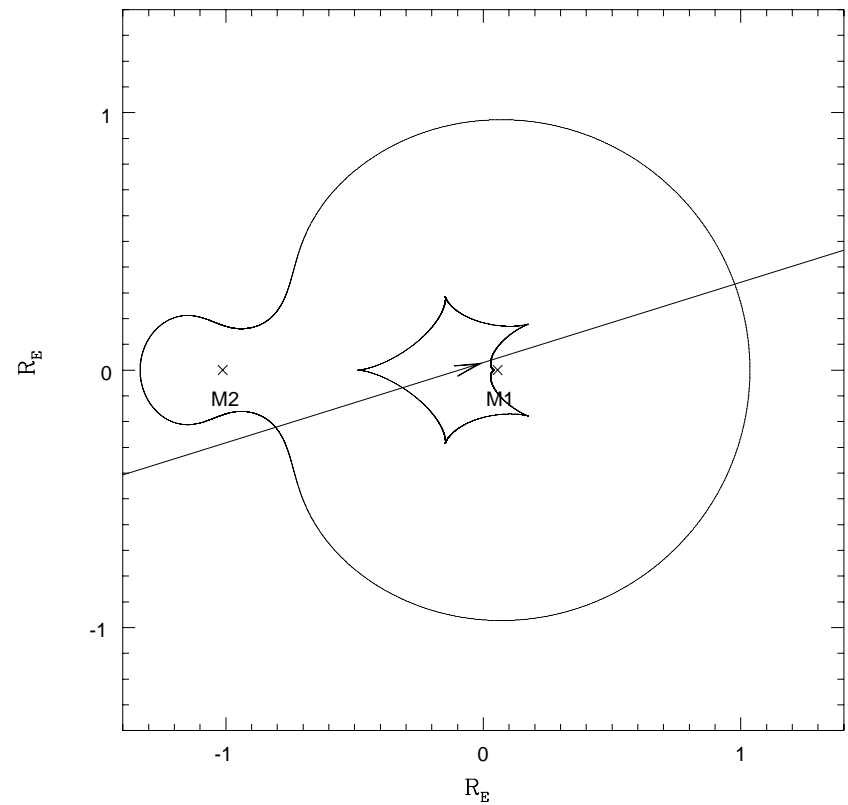

FIG. 15.-Location of the (cuspy) caustic and (smooth) critical curves for the 94-BLG-4 binary lens fit presented in Fig. 14. The coordinate system, whose origin is at the center of mass, indicates distance in units of the system's Einstein Ring radius $R_{\mathrm{E}}$. Also shown are the locations of the lensing objects, and the trajectory of the source through the caustic structure.

the clump. However, the fits suggest only $\sim \frac{1}{5}$ of this light is lensed, and so the source likely resides further down the main sequence as a late A or early F star. The lensed source does appear 0". $4 \pm$ 0". 1 from the MACHO object, based upon DIP analysis.

The first caustic crossing appears to be resolved with two observations on its rise. If we assume the source is not itself a binary, we can estimate the source radius as $1.5 \pm 0.2 R_{\odot}$ from its color and magnitude. The measured $t_{*}=0.65$

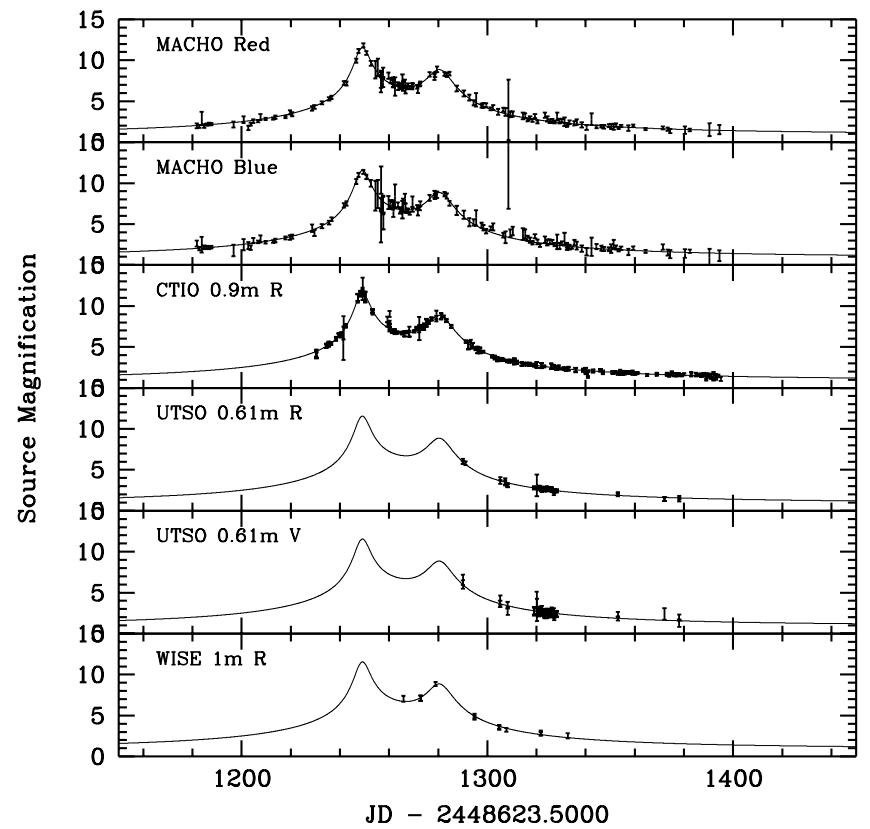

FIG. 16.- Light curve of MACHO event 95-BLG-12, including our fit to binary microlensing. 


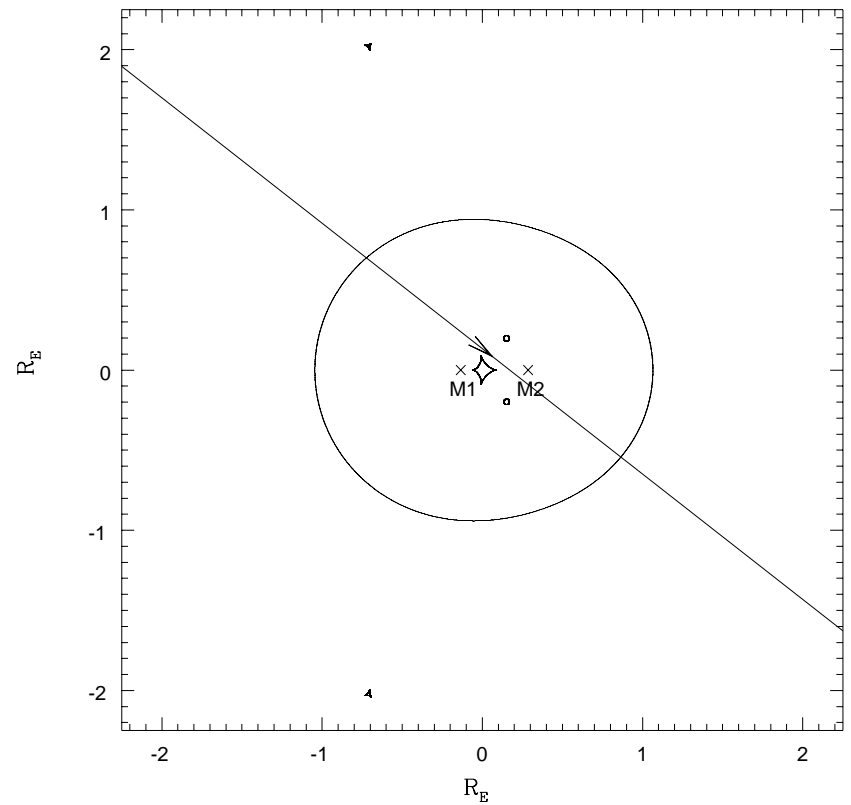

FIG. 17.- Location of the (cuspy) caustic and (smooth) critical curves for the 95-BLG-12 binary lens fit presented in Fig. 16. The coordinate system, whose origin is at the center of mass, indicates distance in units of the system's Einstein Ring radius $R_{\mathrm{E}}$. Also shown are the locations of the lensing objects, and the trajectory of the source through the caustic structure. \pm 0.10 days yields a lens velocity projected to the LMC (assumed to be at $50 \mathrm{kpc}$ ) of $19 \pm 4 \mathrm{~km} \mathrm{~s}^{-1}$, considerably lower than what one would expect from a halo lens. In fact, this velocity is low enough that it is only marginally consistent with a lens in the disk of the LMC and would not be consistent with any LMC models including velocity dispersions in excess of the measured values (Bennett et al. 1996b).

Aubourg et al. (1999) have suggested an LMC model with a self-lensing optical depth large enough to explain the excess of lensing events observed toward the LMC. Aubourg et al. (1999) achieve a high microlensing optical depth without violating the virial relation between velocity dispersion and microlensing optical depth (Gould 1995) by arguing that the velocity dispersion of the lens population is much higher than the velocity dispersion of the stars with measured radial velocities (Cowley \& Hartwick 1991; Zaritsky et al. 1999). However, even in such a model our projected velocity of $19 \pm 4 \mathrm{~km} \mathrm{~s}^{-1}$ for LMC-9 would not be consistent with a lens in the LMC. ${ }^{25}$

Thus, there are two possible interpretations of the LMC-9 caustic crossing observations:

1. They imply that the lens resides in an LMC disk that contains enough mass to generate only a fraction of the

${ }^{25}$ An early version of Aubourg et al. (1999) incorrectly suggested that their model was consistent with the LMC-9 projected velocity (E. Aubourg 1999, private communication).

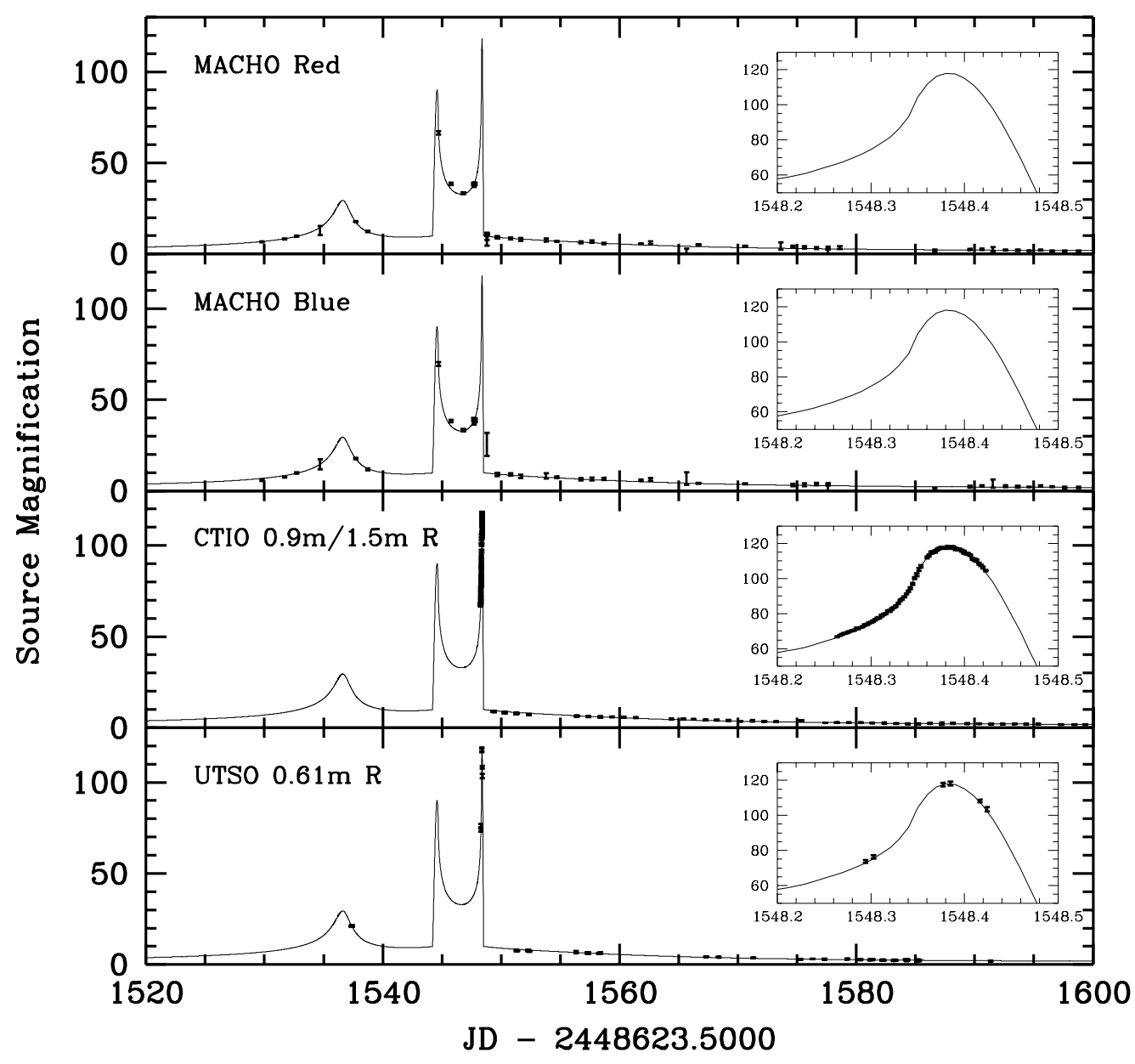

FIG. 18.- Light curve of MACHO event 96-BLG-3, including our fit to binary microlensing 


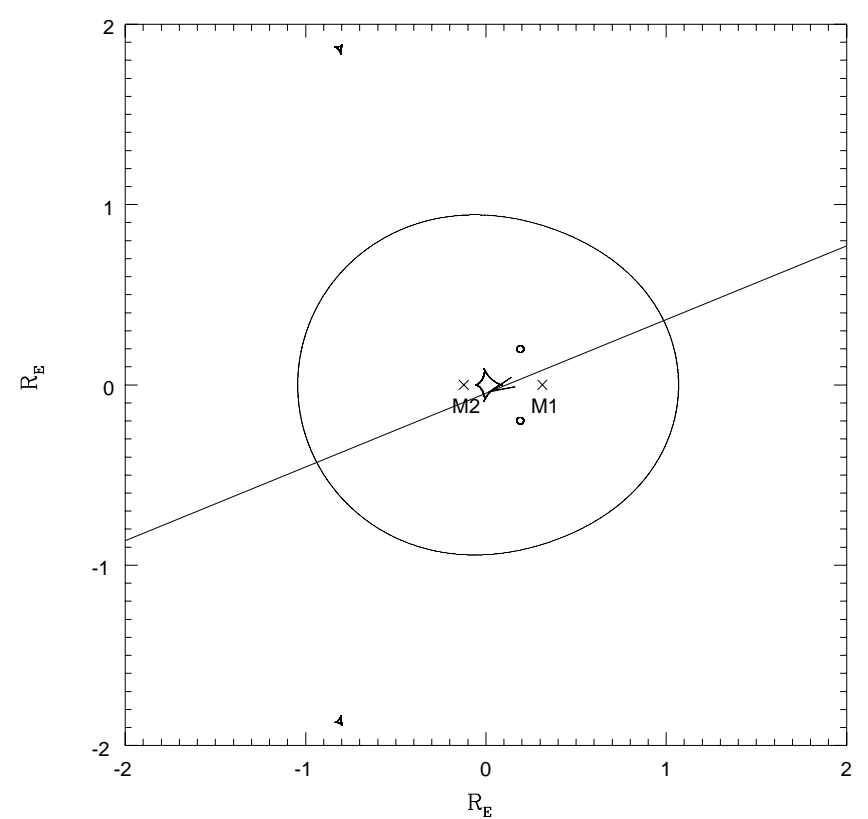

FIG. 19.- Location of the (cuspy) caustic and (smooth) critical curves for the 96-BLG-3 binary lens fit presented in Fig. 18. The coordinate system, whose origin is at the center of mass, indicates distance in units of the system's Einstein Ring radius $R_{\mathrm{E}}$. Also shown are the locations of the lensing objects, and the trajectory of the source through the caustic structure.

observed LMC lensing events, or

2. The source star is itself a binary, and the two caustic crossing observations do not constrain the lens proper motion or its location.

Both of these possibilities have an a priori probability of about $10 \%$. Clearly, better photometric coverage of the caustic crossings could have resolved this issue.

\section{3. $L M C-10$ (18.3324.1765)}

We include LMC-10 as a binary candidate as an example of variability that passes our microlensing cuts (Alcock et al.

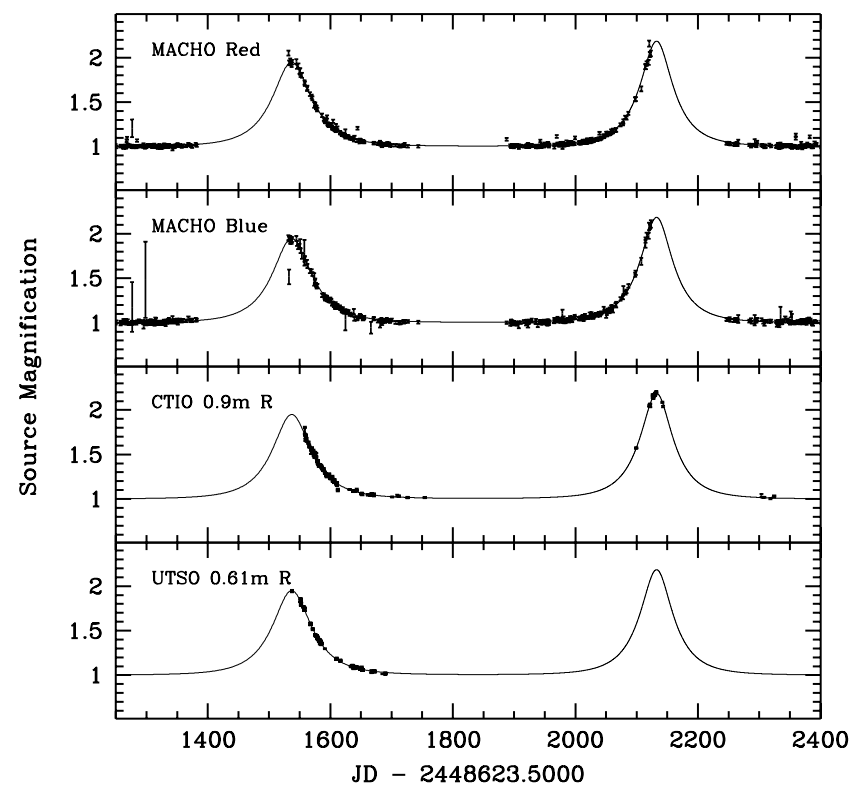

FIG. 20.- - Light curve of MACHO event 96-BLG-4, including our fit to binary microlensing.

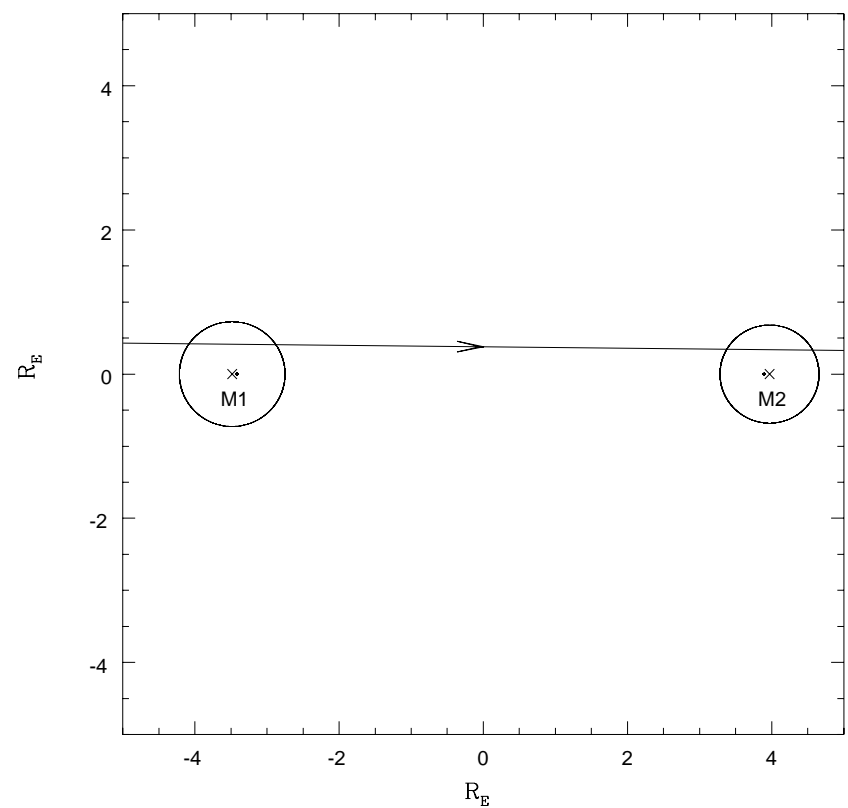

FIG. 21.- Location of the (cuspy) caustic and (smooth) critical curves for the 96-BLG-4 binary lens fit presented in Fig. 20. The coordinate system, whose origin is at the center of mass, indicates distance in units of the system's Einstein Ring radius $R_{\mathrm{E}}$. Also shown are the locations of the lensing objects, and the trajectory of the source through the caustic structure.

1997b) and yet is almost certainly not standard single lens microlensing because of its asymmetry (Fig. 6). Explanations for this event include intrinsic stellar variability (we detect no recurrence of this behavior in five further years of observations), a background supernova (we find no obvious host galaxy down to $R \sim 21$ ), or possibly a weakly perturbed binary microlensing event. Such light curves are thought to compose a significant fraction of all binary lensing events (Di Stefano 1999). The best fit light curve does have a pair of caustic crossings, but they are separated

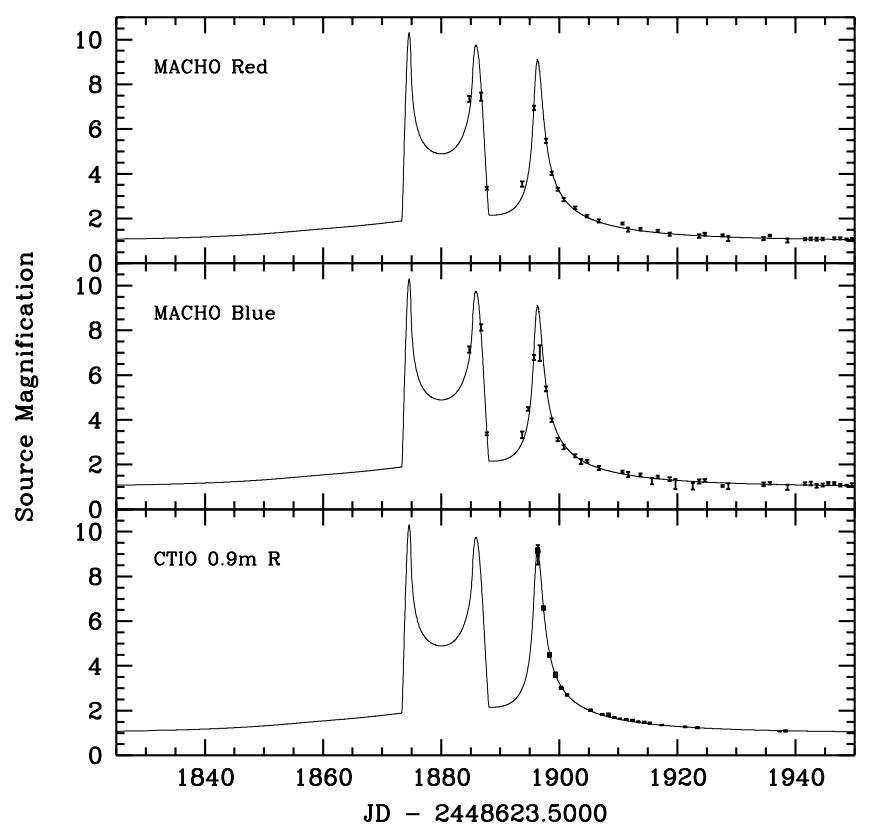

FIG. 22.- Light curve of MACHO event 97-BLG-1, including our fit to binary microlensing. 


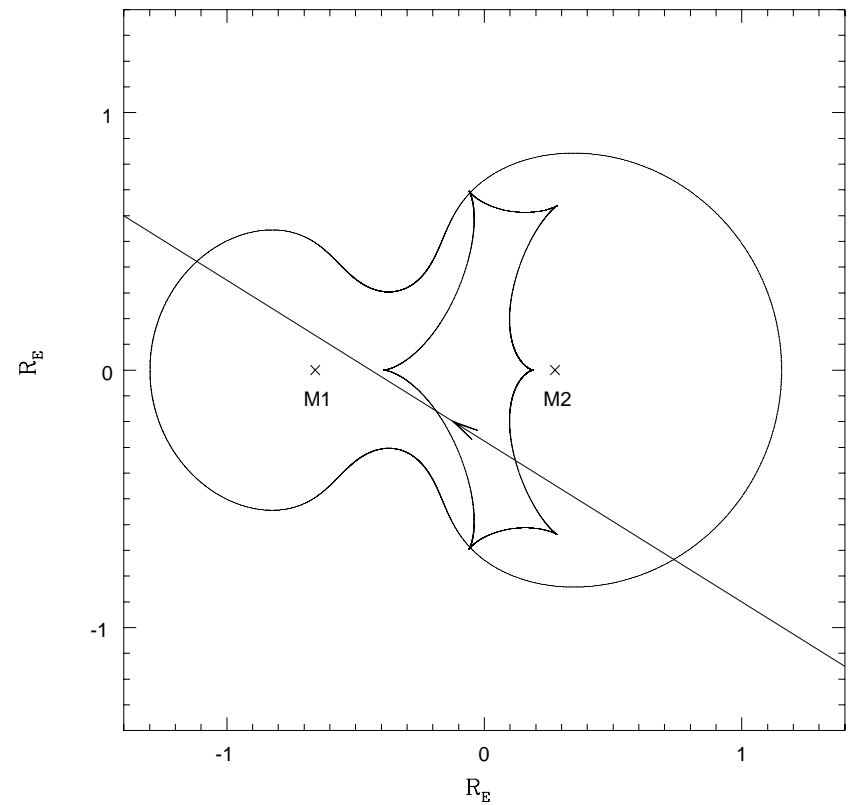

FIG. 23.- Location of the (cuspy) caustic and (smooth) critical curves for the 97-BLG-1 binary lens fit presented in Fig. 22. The coordinate system, whose origin is at the center of mass, indicates distance in units of the system's Einstein Ring radius $R_{\mathrm{E}}$. Also shown are the locations of the lensing objects, and the trajectory of the source through the caustic structure. by about $4 \mathrm{hr}$ and occurred during daylight hours in Australia. For $t_{*}<0.2$ days, these caustic crossings would not have been observable from Australia, and the fit is a plausible explanation of the light curve. The microlensing interpretation of this event would be much more secure if such a light curve were seen in a time reversed order, since the observed rapid rise followed by a slow decay is common in known types of stellar variability. The one observational test that can still be performed is to obtain high-resolution Hubble Space Telescope (HST) images of this object. The centroid of the variable source is well constrained by the DIP method of Tomaney \& Crotts (1996) and appears 1".0 \pm 0 .'2 from the MACHO object. If the binary lens interpretation is correct, then this centroid should be centered on a $R=21.4$ star. Alternatively, the $H S T$ frame might also reveal a background galaxy, which will make the supernova explanation most plausible.

\subsection{8-SMC-1 (208.15683.4237)}

Alert 98-SMC-1, detected on 1998 May 25, was the second microlensing event seen toward the SMC. Nightly GMAN follow-up observations of the early light curve detected evidence of a caustic crossing near June 6.5 UT 1998 (day 2347.5 in Fig. 8; Becker et al. 1998). The prospect of resolving the second caustic crossing, allowing an estimate of the lens location, prompted the microlensing community to commit significant observational resources to

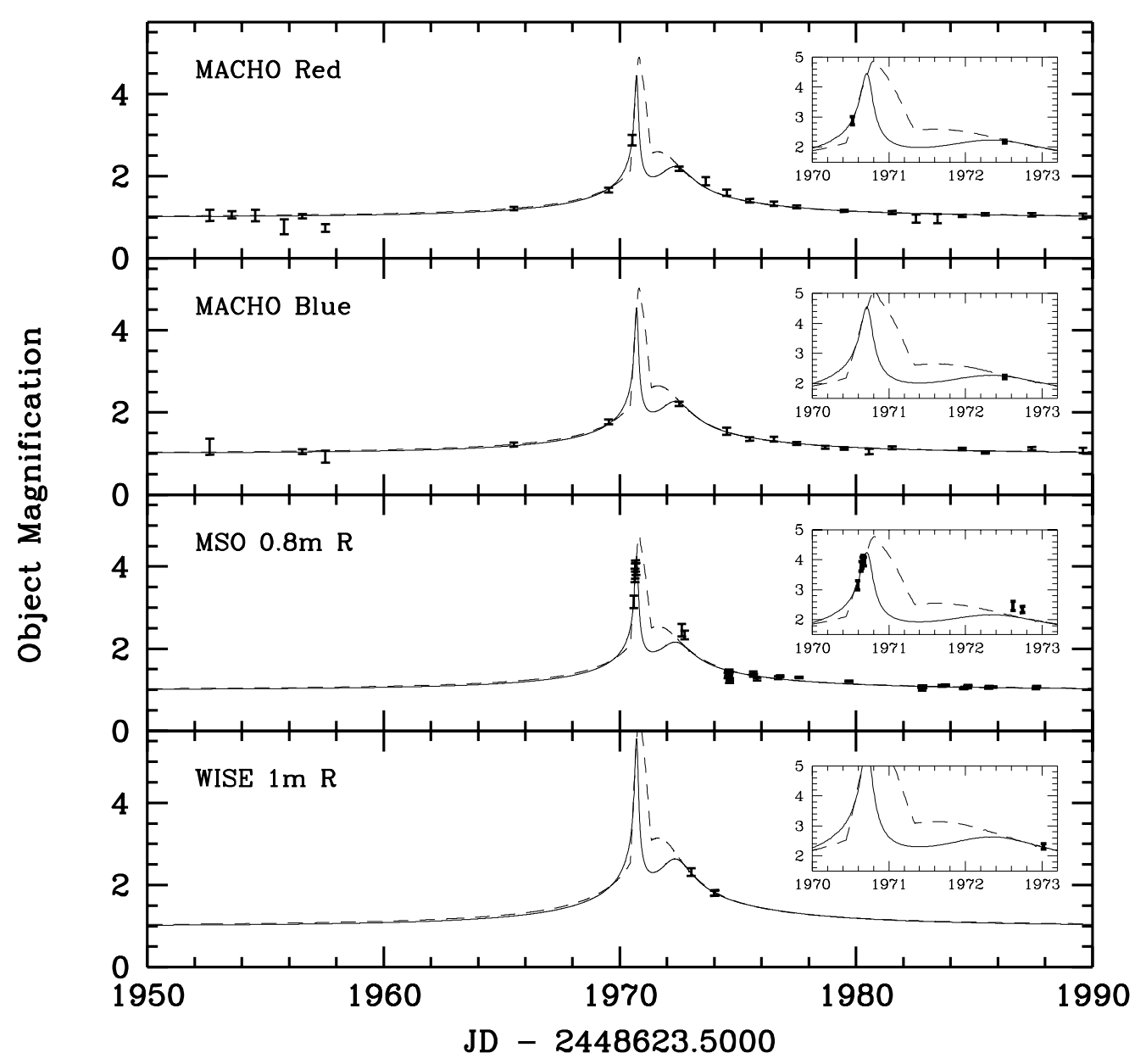

FIG. 24.- Light curve of MACHO event 97-BLG-24, including our fits to binary microlensing. Because of the different blending parameters between fits, we plot the observed magnification of the MACHO object, as opposed to the actual lensed source. 


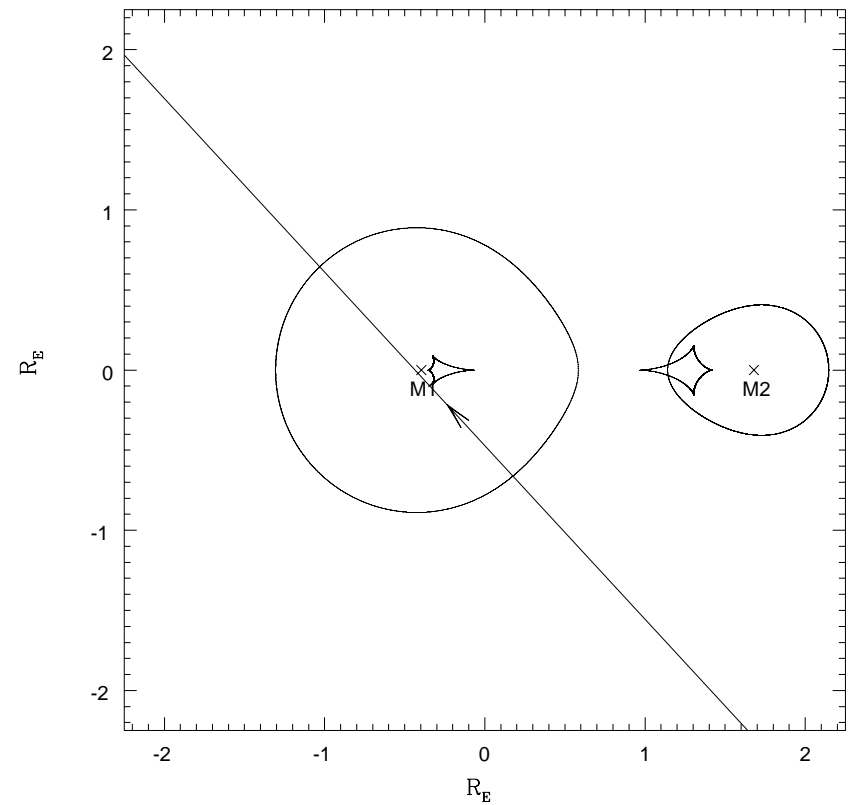

FIG. 25.-Location of the (cuspy) caustic and (smooth) critical curves for the 97-BLG-24 standard binary lens fit (solid line) presented in Fig. 24. The coordinate system, whose origin is at the center of mass, indicates distance in units of the system's Einstein ring radius $R_{\mathrm{E}}$. Also shown are the locations of the lensing objects, and the trajectory of the source through the caustic structure.

this event. Following predictions of the second caustic crossing date by Bennett et al. (1998) and the PLANET collaboration, the event was followed by the EROS (Afonso et al. 1998), PLANET (Albrow et al. 1999a), MACHO/ GMAN (Alcock et al. 1999a), OGLE (Udalski et al. 1998), and MPS collaborations (Rhie et al. 1999). In each case, the

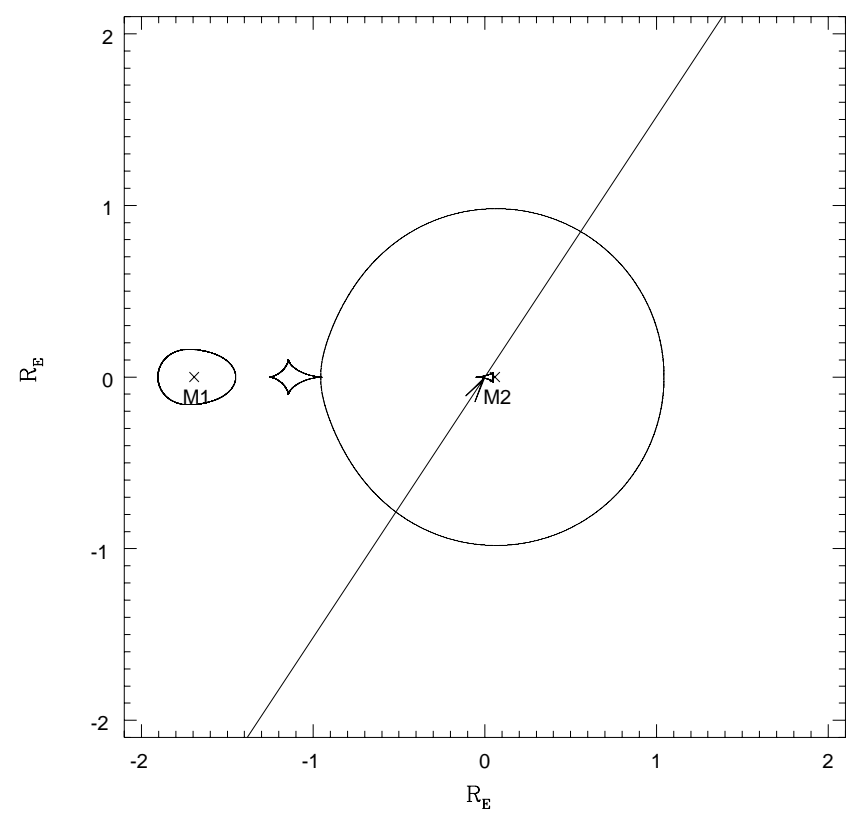

FIG. 26.-Location of the (cuspy) caustic and (smooth) critical curves for the 97-BLG-24 planetary binary lens fit (dashed line) presented in Fig. 24. The coordinate system, whose origin is at the center of mass, indicates distance in units of the system's Einstein ring radius $R_{\mathrm{E}}$. Also shown are the locations of the lensing objects, and the trajectory of the source through the caustic structure. analysis of event data leads to a relatively long caustic crossing timescale, with the robust conclusion that 98-SMC-1 is due to SMC-SMC self lensing. A joint analysis incorporating all event data confirms these results (Afonso et al. 2000).

The fit we present here, originally from Alcock et al. (1999a), exhibits a $t_{*}=0.116 \pm 0.010$ days. We estimate $R_{*}=1.1 \pm 0.1 R_{\odot}$, and assuming the source resides in the SMC at $60 \mathrm{kpc}$ we find a lens velocity projected to the SMC of $\hat{v}=76 \pm 10 \mathrm{~km} \mathrm{~s}^{-1}$. The probability for a Galactic halo lens to produce a $\hat{v}$ value this small is of order $0.1 \%$.

\subsection{OGLE-7/MACHO-119-A (119.20226.2119)}

This caustic crossing event was initially detected by the OGLE collaboration (Udalski et al. 1994) and later verified with data from the MACHO database (Mao et al. 1994). As OGLE-7, it was the first reported case of binary microlensing. 119-A is a moderately blended event, with $f \sim 0.4$, and the source appears to be located near the top of the bulge main sequence.

The fit presented in Figure 10, and the geometry of the lensing encounter in Figure 11, are qualitatively similar to the analysis of Udalski et al. (1994). A comparison of event parameters (MACHO:OGLE) yields $\hat{t}=(169: 160), u_{\min }=$ $(0.08: 0.05), \quad a=(1.05: 1.14), \quad \epsilon_{1}=(0.55: 0.50), \quad$ and $\theta=(-0.94: 0.84)$, where the difference in $\theta$ is due to a different orientation of coordinate systems. Fortunately, the MACHO data set provides better sampling of the light curve and thus more tightly constrains the global fit. The data presented here include two observations on the falling portion of the second caustic crossing, yielding an additional constraint of $t_{*}=0.21 \pm 0.03$ days.

\section{6. $\mathrm{MACHO}-403-\mathrm{C}(403.47793 .2961)$}

Event 403-C exhibits a series of photometric deviations near 1996 August 18 (day 1690 in Fig. 12), which are, for the most part, achromatic. It is plausible these deviations are due to gravitational microlensing. However, this event does not appear to be consistent with single lens microlensing and is only marginally consistent with binary microlensing. The sparse sampling and relatively large error bars prevent tight constraints on the binary microlensing event parameters.

\section{7. $94-B L G-4(118.18141 .731)$}

94-BLG-4 exhibits features similar to LMC-1, with a high- $\sigma$ deviation near the peak of the event, which is unresolved by the survey system. This event was initially reported in Bennett et al. (1997), after discovery during testing of the MACHO Alert System. The two observations apparently taken between caustic crossings are not enough to completely constrain the lensing system; thus Figures 14 and 15 are probably not unique interpretations of this sparsely sampled light curve.

The lensed object is a clump giant, with $V=17.9$, $V-R=1.1$, based upon its position in Figure 46. The binary fit blend fraction of $f \sim 1$ indicates the clump giant itself was lensed, avoiding fit ambiguities introduced by unknown or unconstrained blend fractions, which directly influence our measurement of $\hat{t}$. The event duration is relatively short, $\hat{t}=10.7$ days. If we assume "typical" parameters for the lensing object $\left(v_{\perp}=150 \mathrm{~km} \mathrm{~s}^{-1}\right.$ and a distance $80 \%$ of the way to the Galactic bulge), we arrive at the 


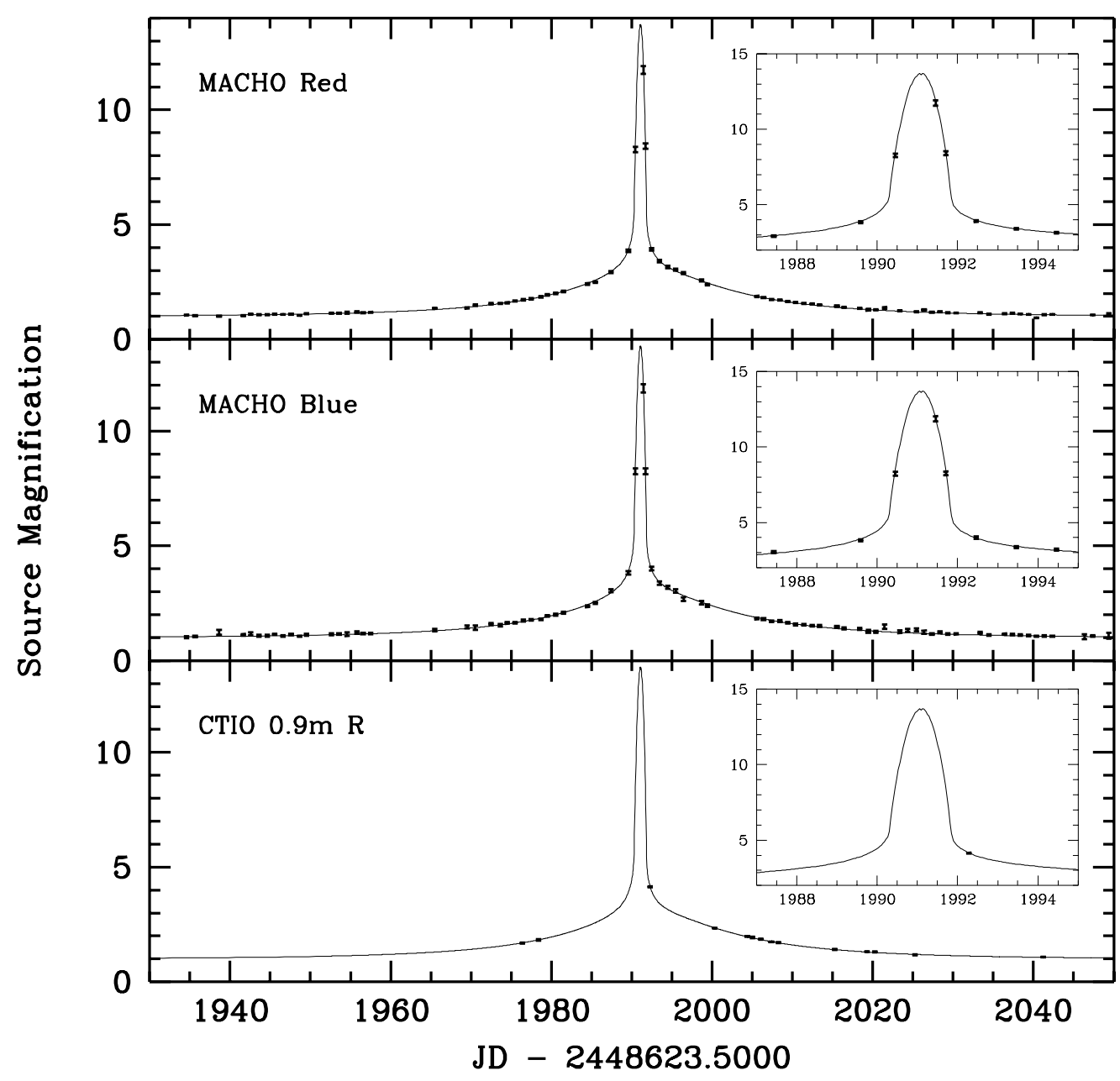

FIG. 27.- Light curve of MACHO event 97-BLG-28, including our fit to binary microlensing

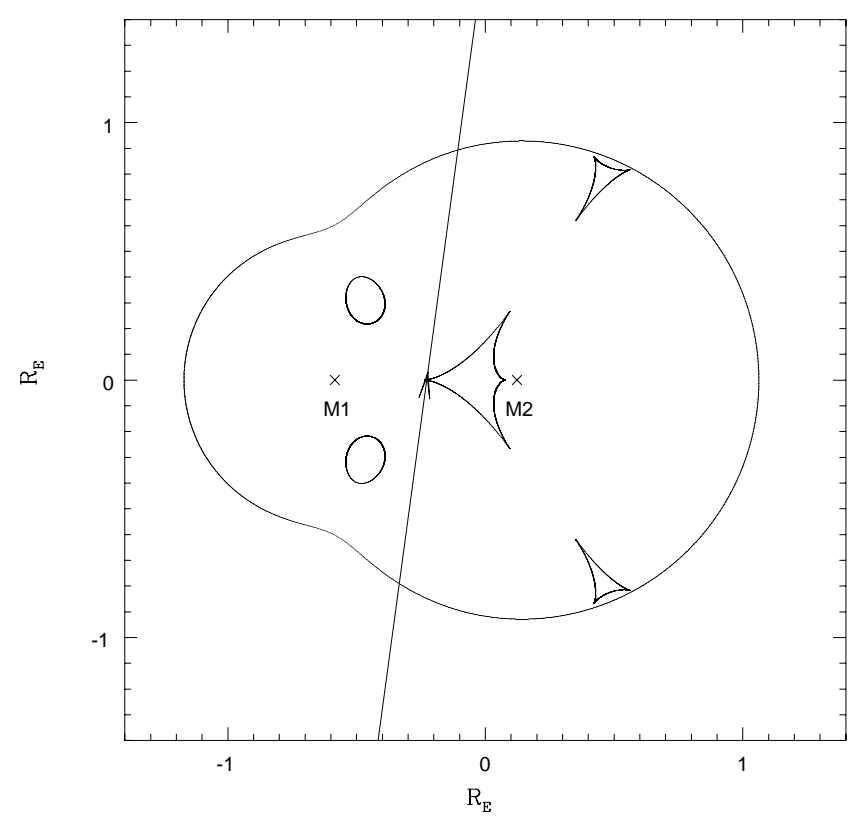

FIG. 28. - Location of the (cuspy) caustic and (smooth) critical curves for the 97-BLG-28 binary lens fit presented in Fig. 27. The coordinate system, whose origin is at the center of mass, indicates distance in units of the system's Einstein Ring radius $R_{\mathrm{E}}$. Also shown are the locations of the lensing objects, and the trajectory of the source through the caustic structure.

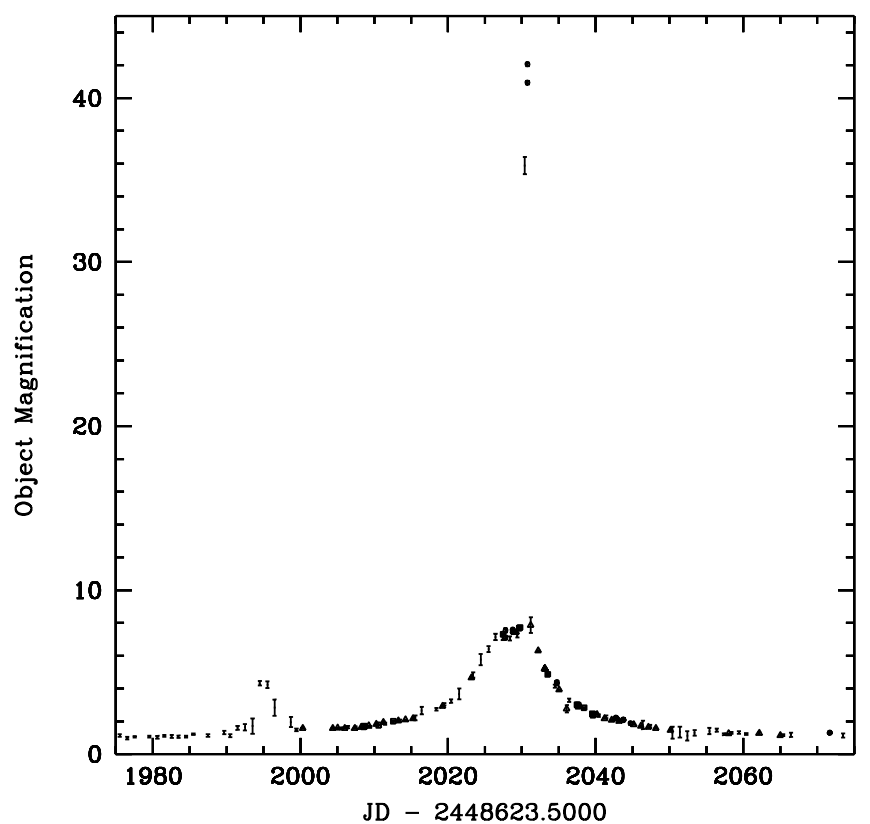

FIG. 29.-Light curve of MACHO event 97-BLG-41, with an approximate baseline determined from a fit to the second peak, disregarding the caustic features. Plotted are the MACHO-B ( points), CTIO-r (triangles), MSO30-r ( squares) and WISE-r ( circles) data. 


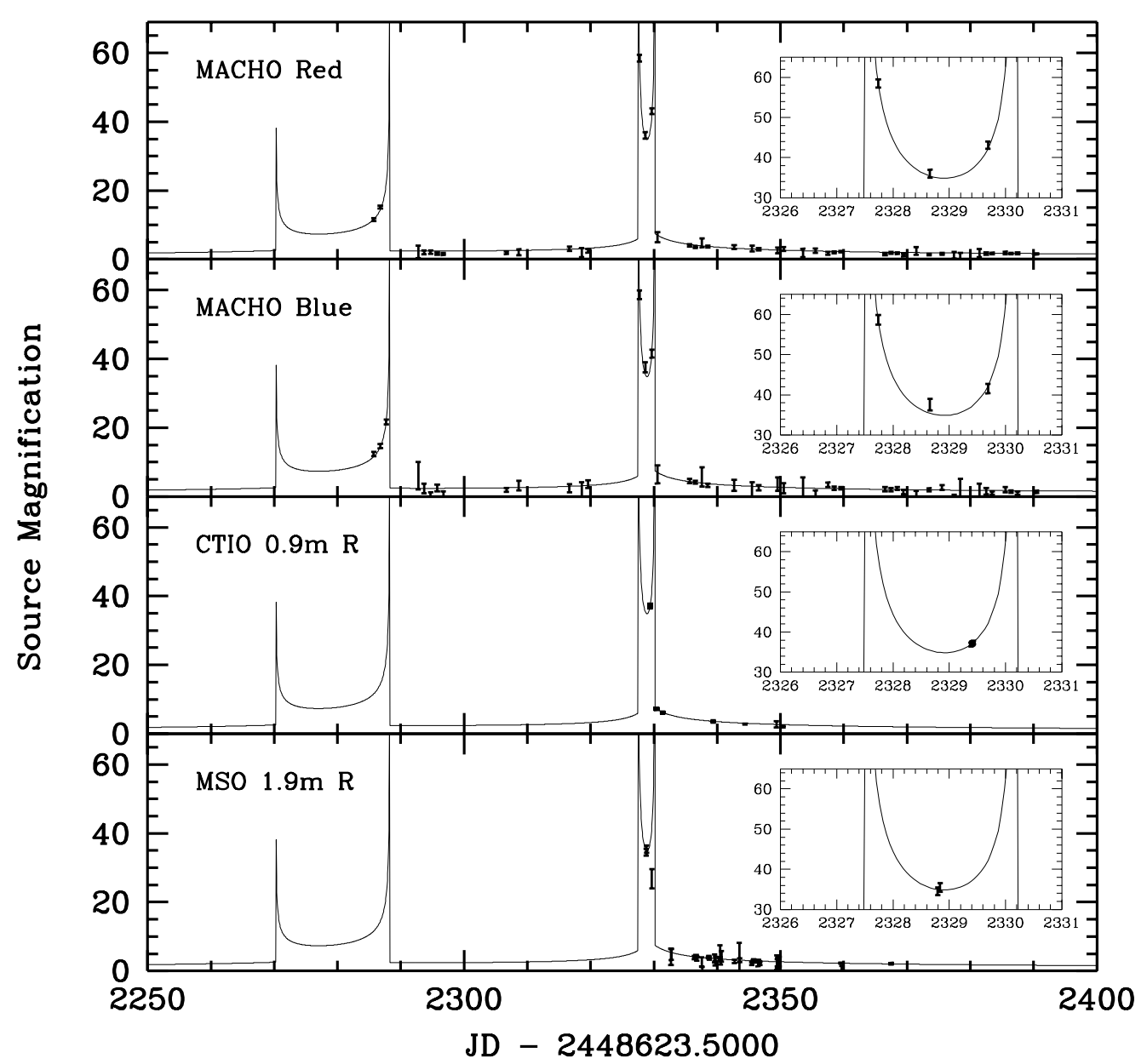

FIG. 30.- Light curve of MACHO event 98-BLG-12, including our fit to binary microlensing

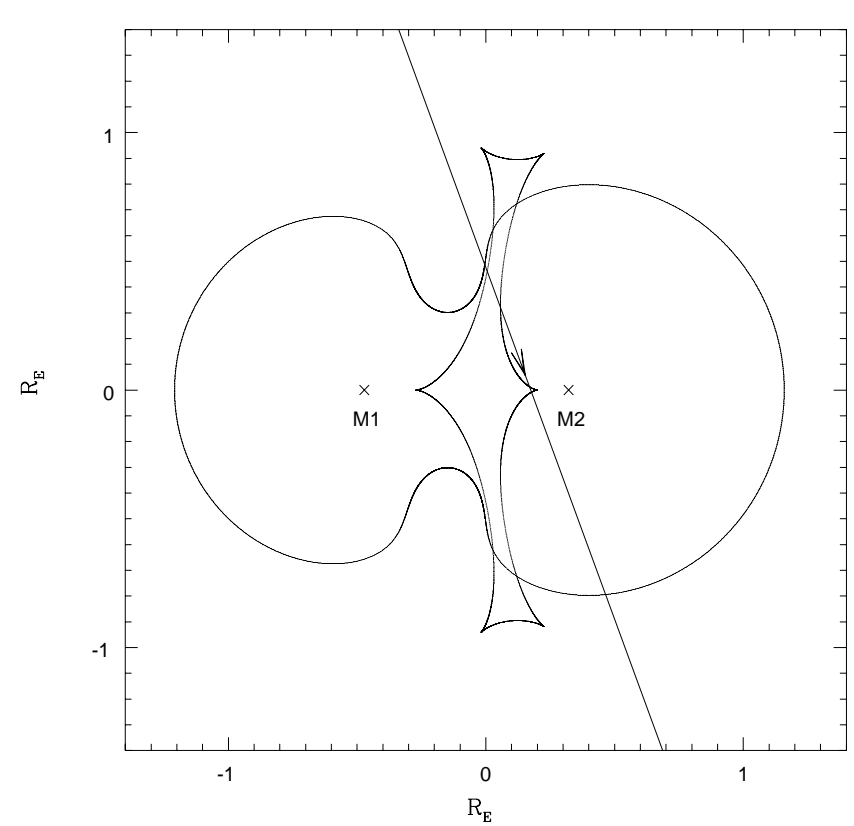

FIG. 31.- Location of the (cuspy) caustic and (smooth) critical curves for the 98-BLG-12 binary lens fit presented in Fig. 30. The coordinate system, whose origin is at the center of mass, indicates distance in units of the system's Einstein Ring radius $R_{\mathrm{E}}$. Also shown are the locations of the lensing objects, and the trajectory of the source through the caustic structure. relationship $\hat{t} \sim 70\left(m / M_{\odot}\right)^{1 / 2}$ days (Paczyński 1991). This implies an overall lens mass of $0.02 M_{\odot}$-however, we expect the shortest of our events to be drawn from lenses residing closer to the source, so the actual mass is likely to be much larger than this. If we take an upper limit of 0.2 $M_{\odot}$ as the total lens mass, then the mass ratio of $1 / 18$ implies a secondary lens of $\lesssim 10$ Jupiter masses for this particular fit.

\subsection{5-BLG-12 (120.21263.1213)}

This twelfth Alert of the 1995 bulge season was detected on May 15, at a magnification of $A \sim 2$. Real-time followup observations by both GMAN (Pratt et al. 1996) and PLANET (Albrow et al. 1996) detected deviations from the standard fit near June 5, making 95-BLG-12 the first binary event detected in real time. Data on this event from the PLANET collaboration are presented in Albrow et al. (1998a).

The lensed object is located on the subgiant branch of the bulge, below the clump. However, because subgiants are rare, we expect that the majority of objects in this location of the color-magnitude diagram are actually blends of multiple fainter stars. Thus, it is no surprise that the binary lens fit indicates blend fractions of $\sim 0.2-0.3$. 95-BLG-12 is a good example of a significantly perturbed, noncaustic crossing binary lensing event (Fig. 16). The multiple peaks are caused by the source approaching cusps in the caustic curve, as displayed in Figure 17. The extensive follow-up 


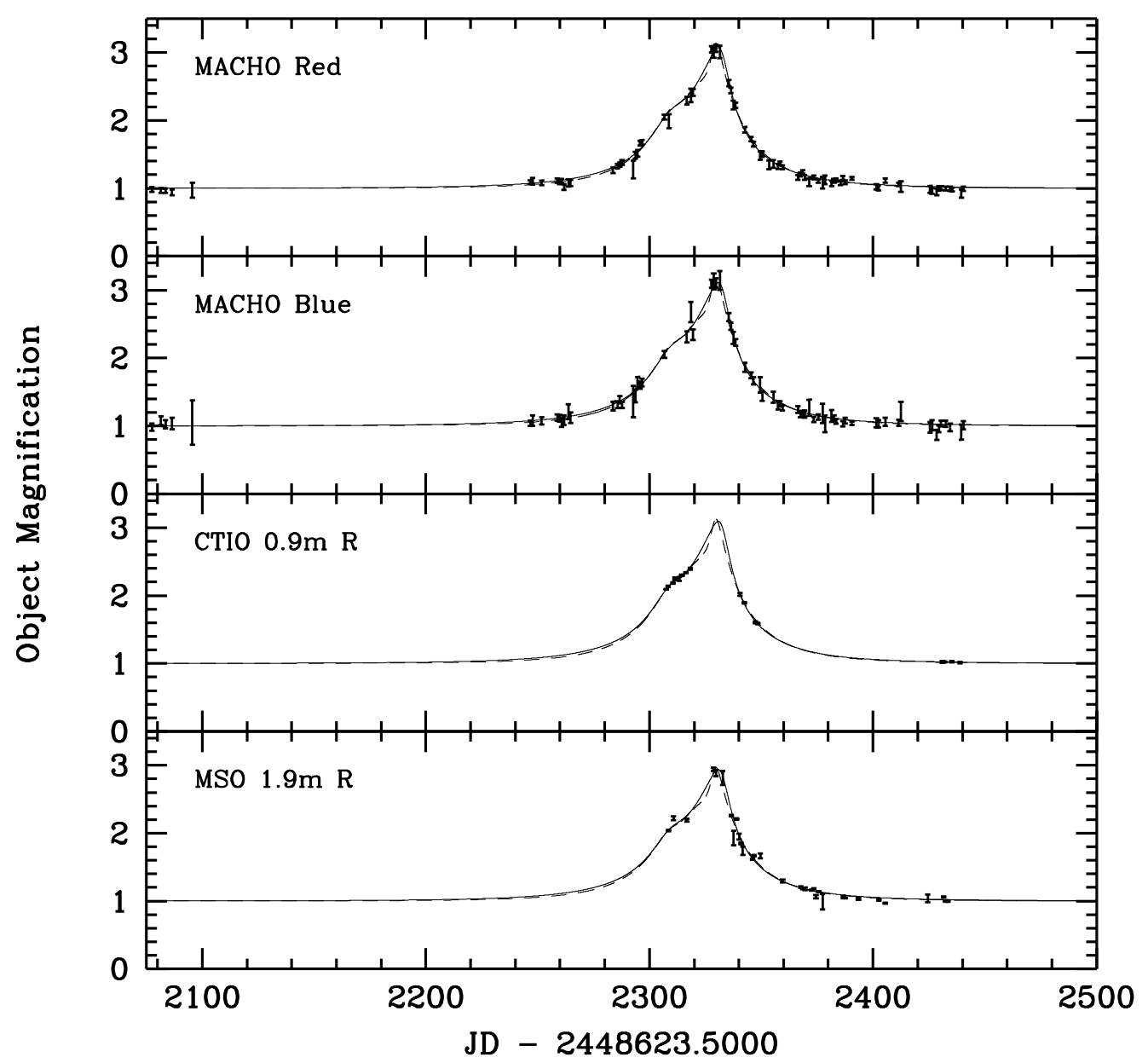

FIG. 32.-Light curve of MACHO event 98-BLG-14, including our fits to binary microlensing. Because of the different blending parameters between fits, we plot the observed magnification of the MACHO object, as opposed to the actual lensed source. The MSO74 data have been averaged into 1 day bins.

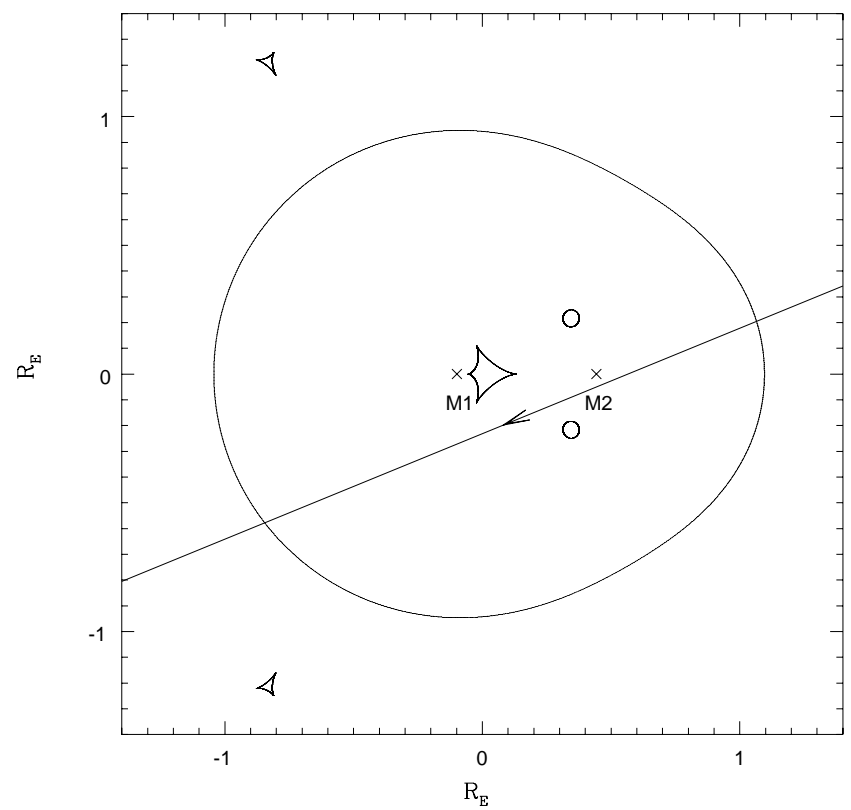

FIG. 33.- Location of the (cuspy) caustic and (smooth) critical curves for the solid 98-BLG-14 binary lens fit (solid line) presented in Fig. 32. The coordinate system, whose origin is at the center of mass, indicates distance in units of the system's Einstein Ring radius $R_{\mathrm{E}}$. Also shown are the locations of the lensing objects, and the trajectory of the source through the caustic structure. data are able to constrain the event at nearly the $1 \%$ level for much of its duration.

\section{9. $96-B L G-3(119.19444 .2055)$}

This event was discovered at the beginning of the 1996 bulge season, and announced by the MACHO Alert System on 1996 March 12. After an initial peak (due to a cusp approach) that resembled a normal lensing event, the star jumped to a large magnification on 1996 March 25, implying a caustic crossing had likely occurred. Based upon the available MACHO data, Bennett et al. (1996a) were able to successfully predict the second caustic crossing to within 0.15 days. The important features at the time of this prediction were the initial peak in Figure 18, due to a cusp approach, and the sparsely sampled $U$ shape between caustics. These global constraints on the light curve provided enough leverage for an accurate caustic crossing prediction, although this is not necessarily possible with more local constraints, even a well-sampled caustic crossing (Albrow et al. 1999b, 1999c).

Follow-up data taken at the CTIO $1.5 \mathrm{~m}$ provided the first ever resolution of a binary caustic crossing, as shown in Figure 18. In addition, spectroscopic observations of the object were taken during the crossing (Lennon et al. 1996). As the source crossed out of the caustic region, its brightness peaked at extremely high magnification, $A_{\max } \sim 120$. However, the observed magnification of the MACHO 


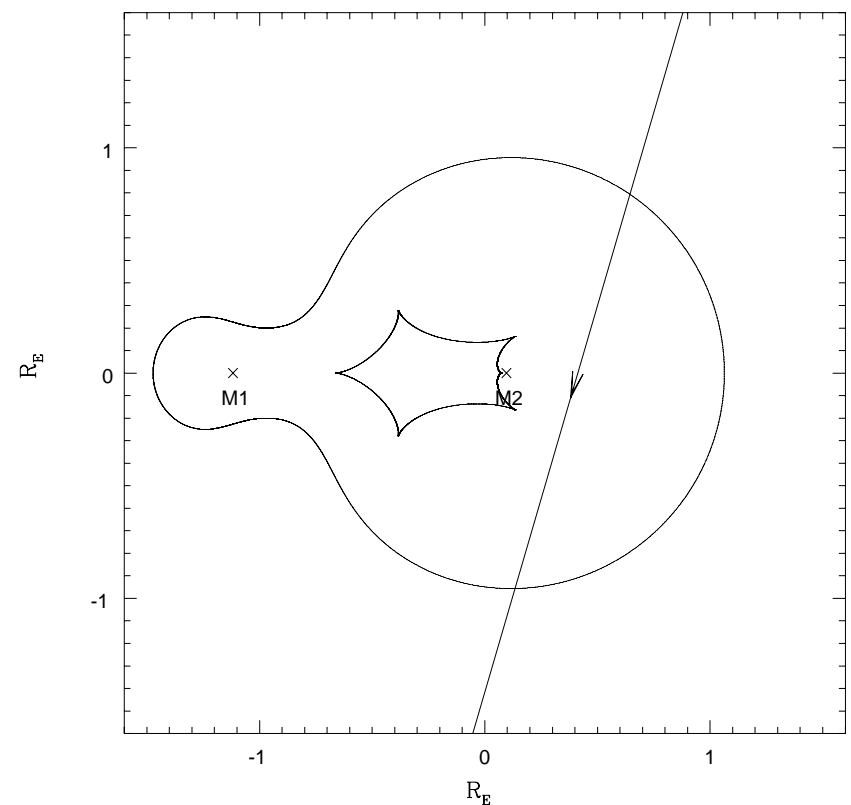

FIG. 34.- Location of the (cuspy) caustic and (smooth) critical curves for the dashed (large mass ratio) 98-BLG-14 binary lens fit (dashed line) presented in Fig. 32. The coordinate system, whose origin is at the center of mass, indicates distance in units of the system's Einstein Ring radius $R_{\mathrm{E}}$. Also shown are the locations of the lensing objects, and the trajectory of the source through the caustic structure.

object was considerably less, as it is a significant blend ( $f \sim 0.2$ for MACHO). Thus, while Lennon et al. (1996) report their spectrum of that of a G0 subgiant, the blend fractions indicate that the source is a G0 dwarf.

Lennon et al. (1996) showed that the source is not a spectroscopic binary and estimated from their spectra an effective temperature of $T_{\text {eff }}=6100 \mathrm{~K}$. Their comparison to evolutionary tracks leads to an angular source radius of $\theta_{*}=0.94 \mu$ as. Since these spectra were taken while the

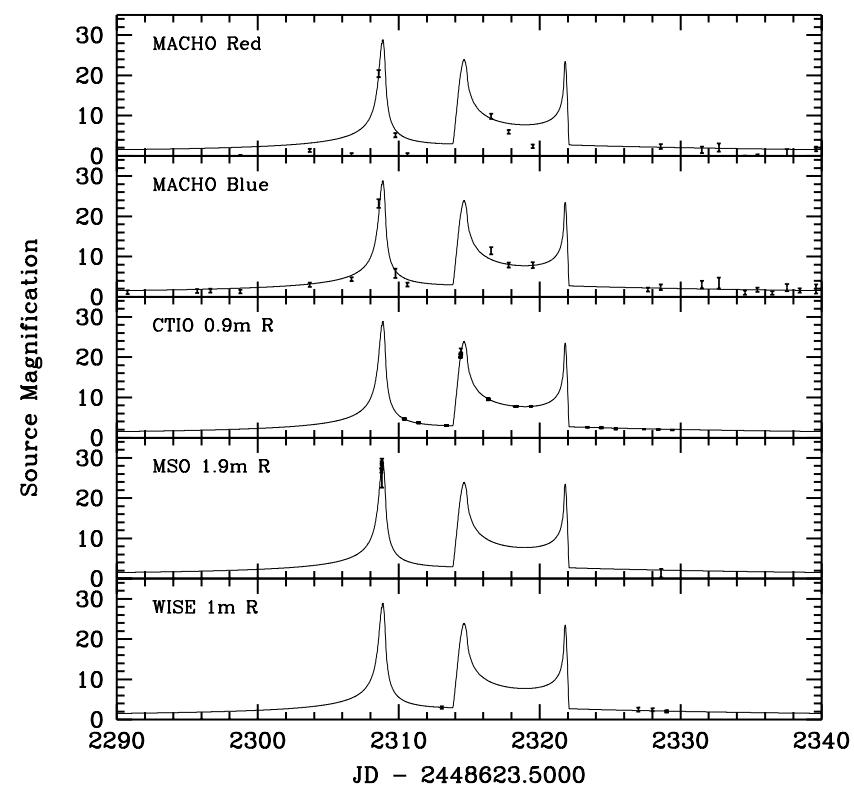

FIG. 35.- - Light curve of MACHO event 98-BLG-16, including our fit to binary microlensing. The MACHO Red data exhibit excessive scatter because of a defective amplifier.

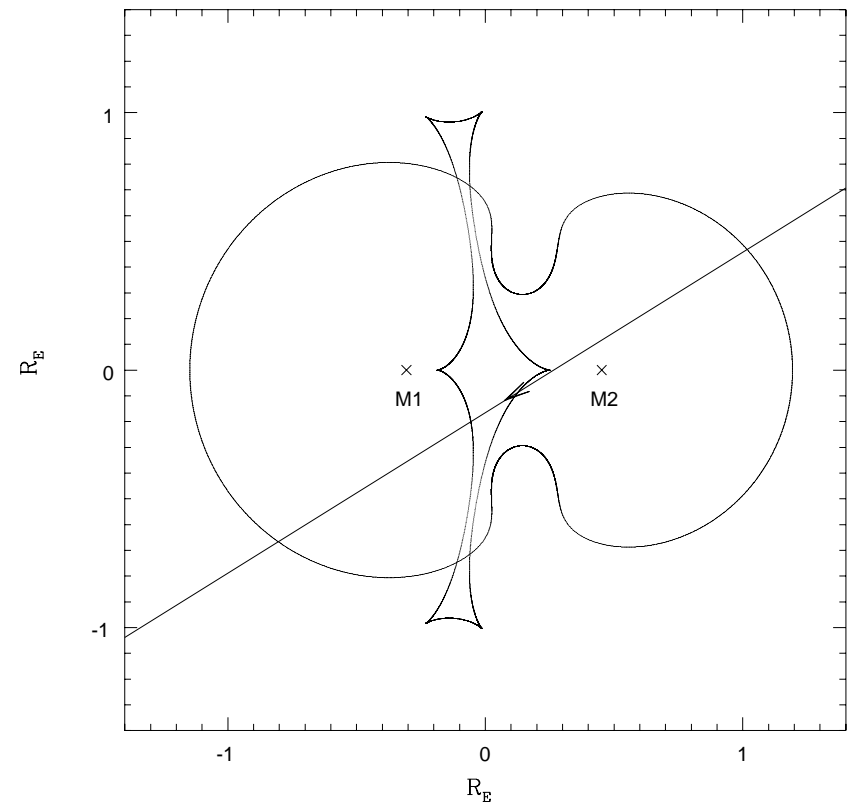

FIG. 36.- Location of the (cuspy) caustic and (smooth) critical curves for the 98-BLG-16 binary lens fit presented in Fig. 35. The coordinate system, whose origin is at the center of mass, indicates distance in units of the system's Einstein Ring radius $R_{\mathrm{E}}$. Also shown are the locations of the lensing objects, and the trajectory of the source through the caustic structure.

source was highly magnified, their $T_{\text {eff }}$ should represent the temperature of the lensed source. However, their estimate of the distance to the source, and hence its angular size, depended upon the baseline brightness of the object $(V=19.2)$, instead of the recovered brightness of the lensed source $(V=20.8)$. Our analysis in $\S 5.2 .2$ and Table 4 indicates our estimate of $T_{\text {eff }}=6200 \mathrm{~K}$ is in excellent agreement with Lennon et al. (1996), and deblending the event reasonably leads to a smaller $\theta_{*}=0.53 \mu$ as.

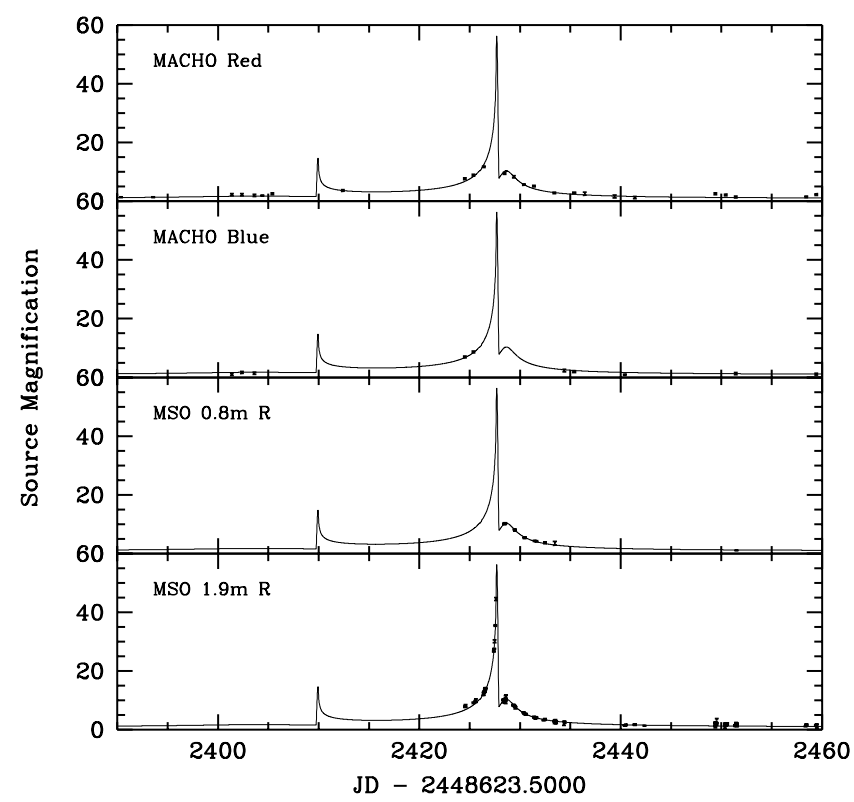

FIG. 37.- - Light curve of MACHO event 98-BLG-42, including our fit to binary microlensing. 


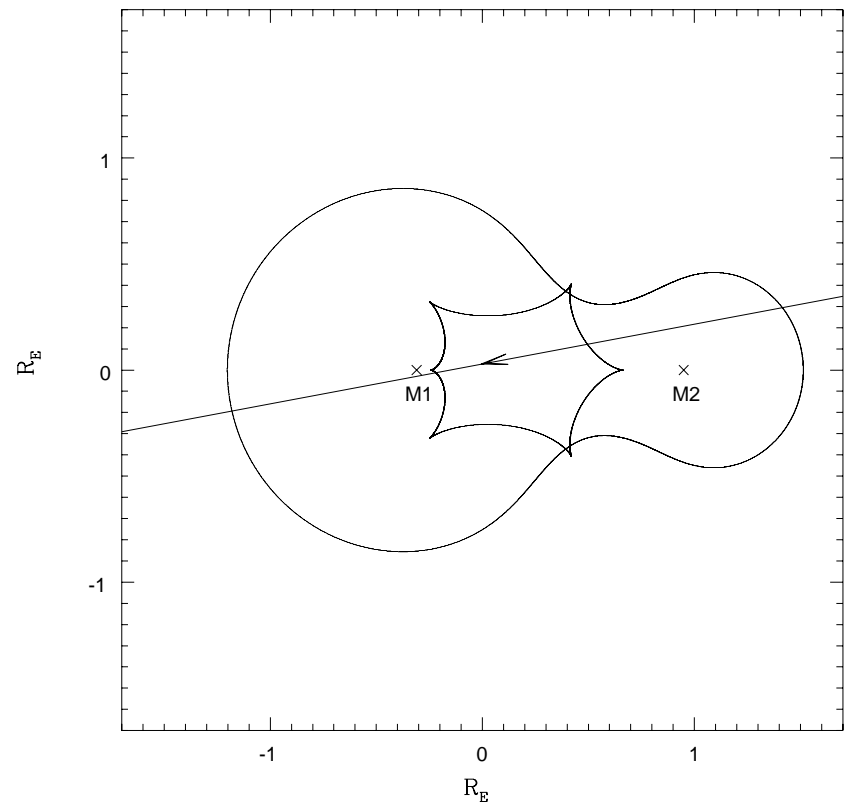

FIG. 38.- Location of the (cuspy) caustic and (smooth) critical curves for the 98-BLG-42 binary lens fit presented in Fig. 37. The coordinate system, whose origin is at the center of mass, indicates distance in units of the system's Einstein Ring radius $R_{\mathrm{E}}$. Also shown are the locations of the lensing objects, and the trajectory of the source through the caustic structure.
4.10. 96-BLG-4 (105.21417.101)

96-BLG-4 displays a repeating variability (Fig. 20), which would generally exclude it as a microlensing candidate. The first peak is well fit by standard microlensing $\left(\chi^{2} /\right.$ dof $\left.=1.2\right)$ and was not a binary candidate until the MACHO Alert System retriggered on this event $\sim 550$ days after the first peak. The color of this object $(V=16.2, V-R=1.1)$ indicates it is unlikely to be a long period variable (LPV), and any periodic variability is ruled out by prior observations. The object does appear to be a bright giant, located close to the tip of the red giant branch.

As is the case with many of our giant sources, we do not detect a significant color shift during the event, and the binary lens fit is consistent with zero blending $(f=1)$. The multiple achromatic peaks suggest lensing of a single giant source by a binary lens, or lensing of a binary system by a single lens. Figure 46 indicates that the source appears $\sim 1.3$ mag brighter than the red clump in this region, so a single lens, binary source event seems plausible (the probability of a chance superposition of giant stars is extremely small). Stars generally spend only a few percent of their lives on the giant branch (Iben 1967), so it is unlikely for two members of a binary system to reside on the giant branch at the same time. However, given the sample of $\sim 350$ candidate events that our binary lens sample has been selected from, one binary giant source event is plausible.

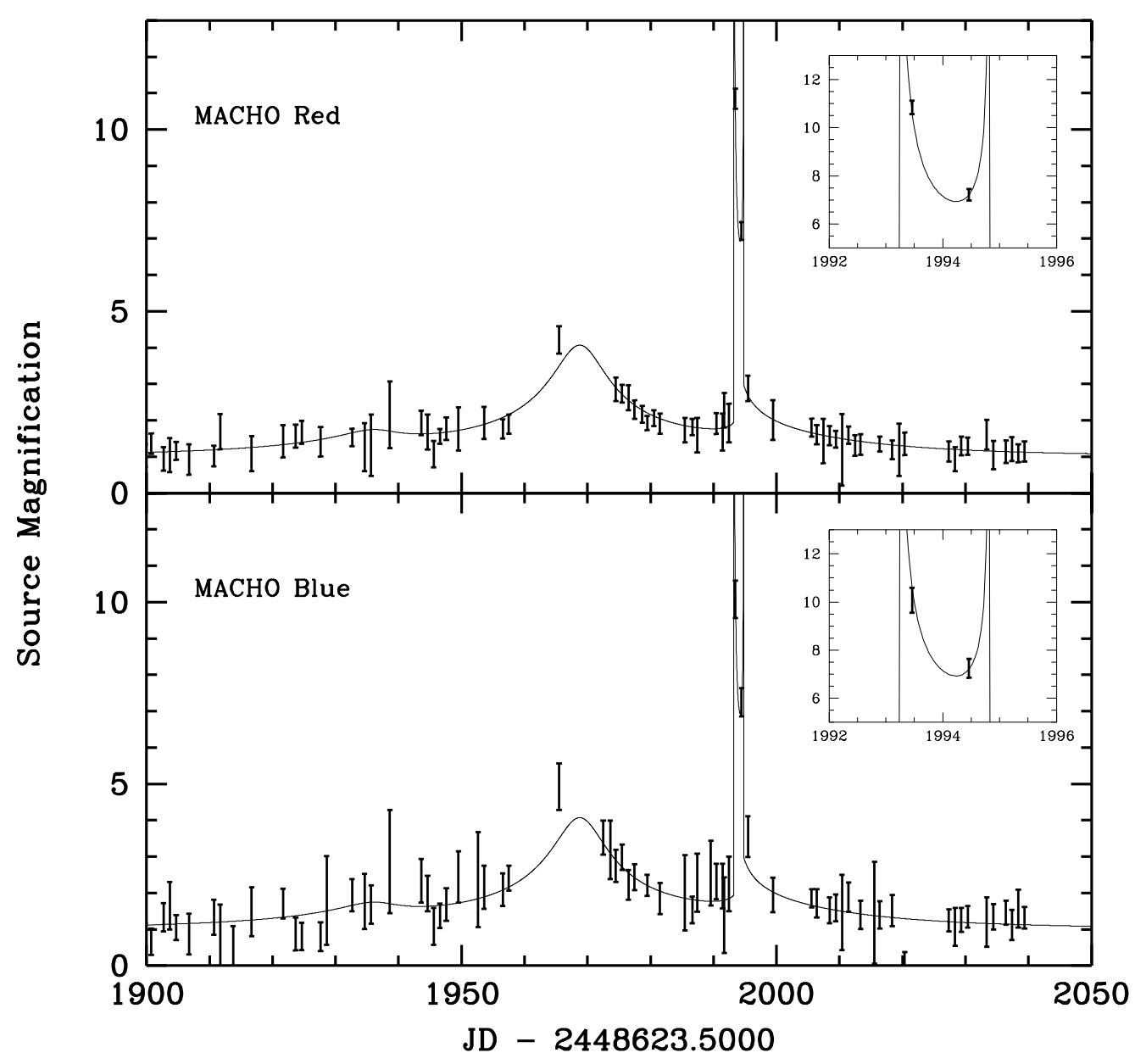

FIG. 39.- - Light curve of MACHO event 97-BLG-d2, including our fit to binary microlensing 


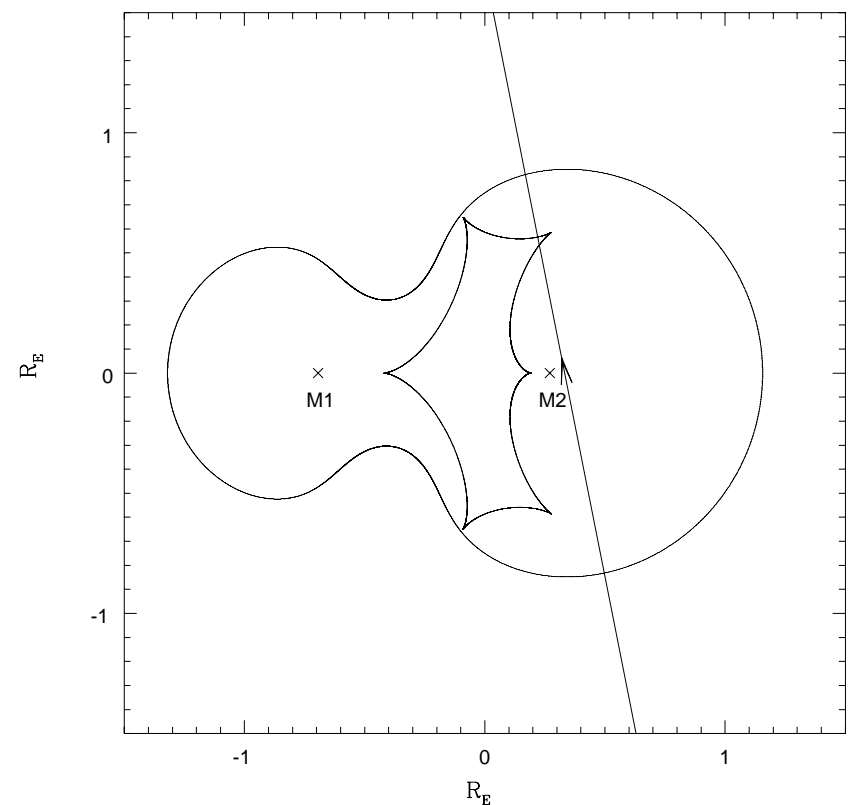

FIG. 40.- Location of the (cuspy) caustic and (smooth) critical curves for the 97-BLG-d2 binary lens fit presented in Fig. 39. The coordinate system, whose origin is at the center of mass, indicates distance in units of the system's Einstein Ring radius $R_{\mathrm{E}}$. Also shown are the locations of the lensing objects, and the trajectory of the source through the caustic structure.

Another interpretation of this event is a very widely separated $\left(a=7.5 R_{\mathrm{E}}\right)$ binary lens acting upon a single background giant source. It has been recognized by Di Stefano \& Mao (1996) that lensing by widely separated binary systems $\left(a>2.5 R_{\mathrm{E}}\right)$ should occur with $\sim 10 \%$ the frequency of close binary events. As shown in Figure 21, the caustics for this event are extremely small, which indicates that this event, unlike any of the others presented in this paper, is very much like the superposition of two single lens light curves. This makes it difficult to discriminate unam-

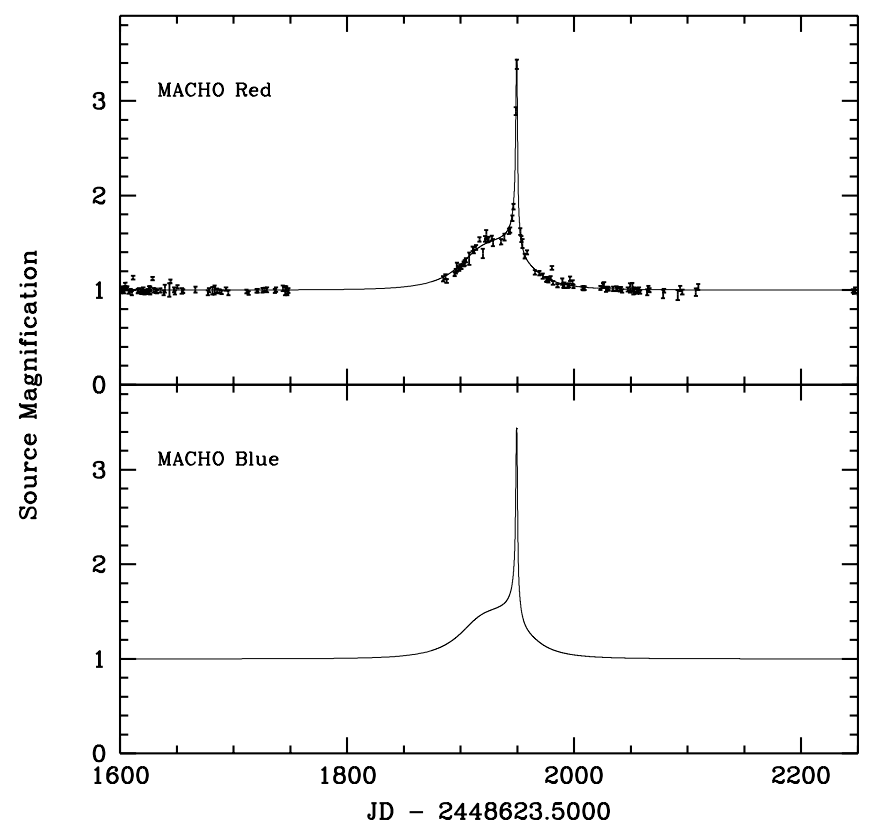

FIG. 41.- Light curve of MACHO event 108-E, including our fit to binary microlensing.

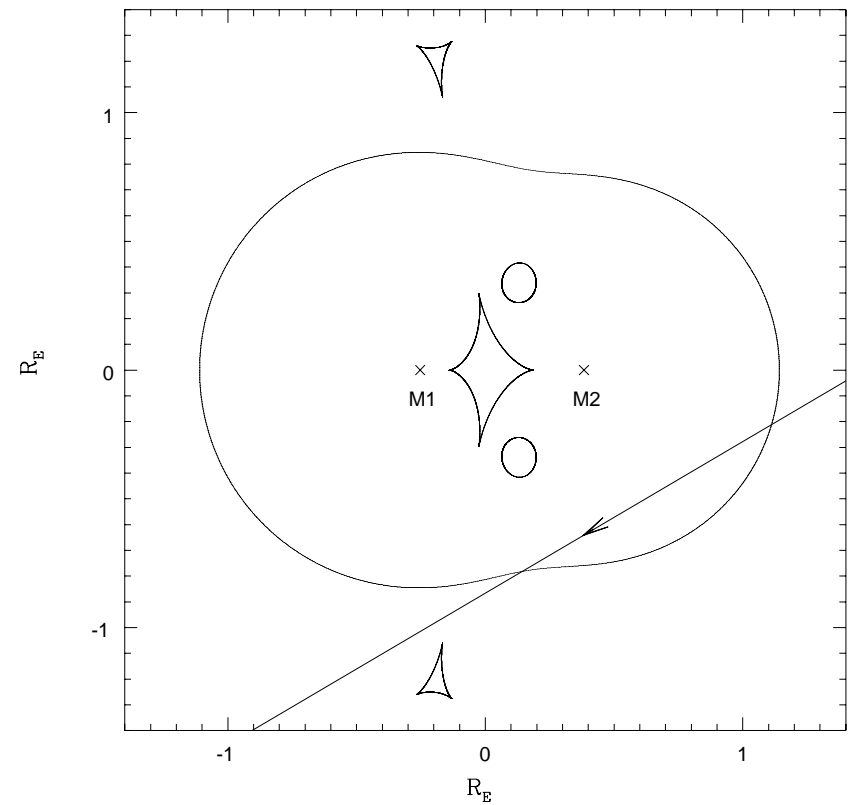

FIG. 42.-Location of the (cuspy) caustic and (smooth) critical curves for the 108-E binary lens fit presented in Fig. 41. The coordinate system, whose origin is at the center of mass, indicates distance in units of the system's Einstein Ring radius $R_{\mathrm{E}}$. Also shown are the locations of the lensing objects, and the trajectory of the source through the caustic structure.

biguously between binary lens and binary source models for this event.

\subsection{7-BLG-1 (113.18674.756)}

97-BLG-1 was initially announced as microlensing on 1997 March 3. A substantial deviation from standard microlensing was noticed 1997 March 11. This sharp decline, seen in Figure 22, signified the source exiting the caustic region, and follow-up efforts were able to sample only the final cusp approach (see Fig. 23).

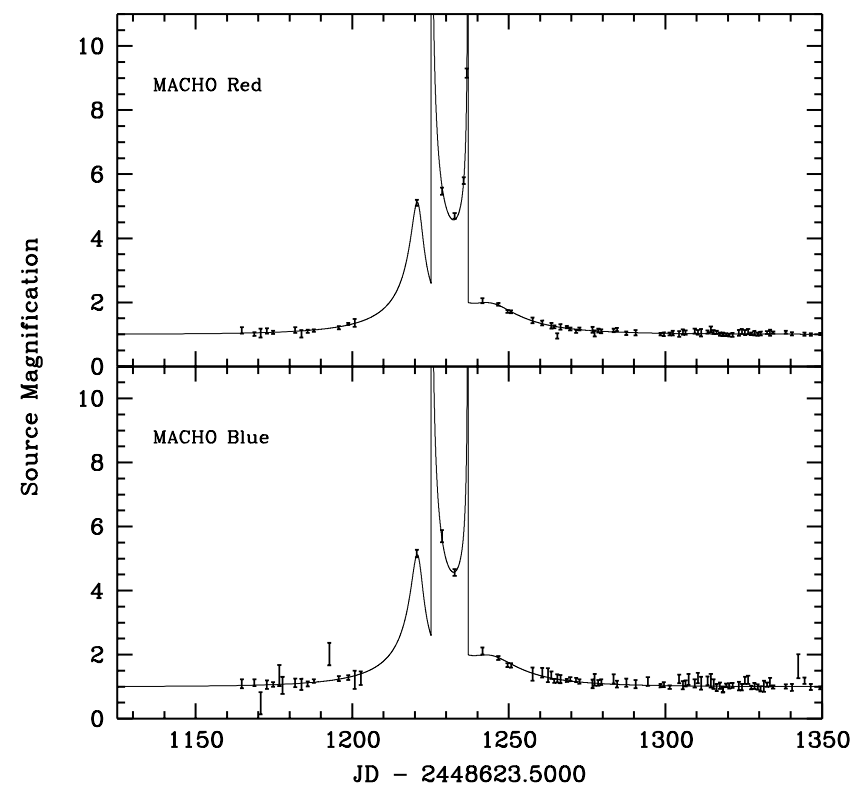

FIG. 43.- Light curve of MACHO event 176-A, including our fit to binary microlensing. 


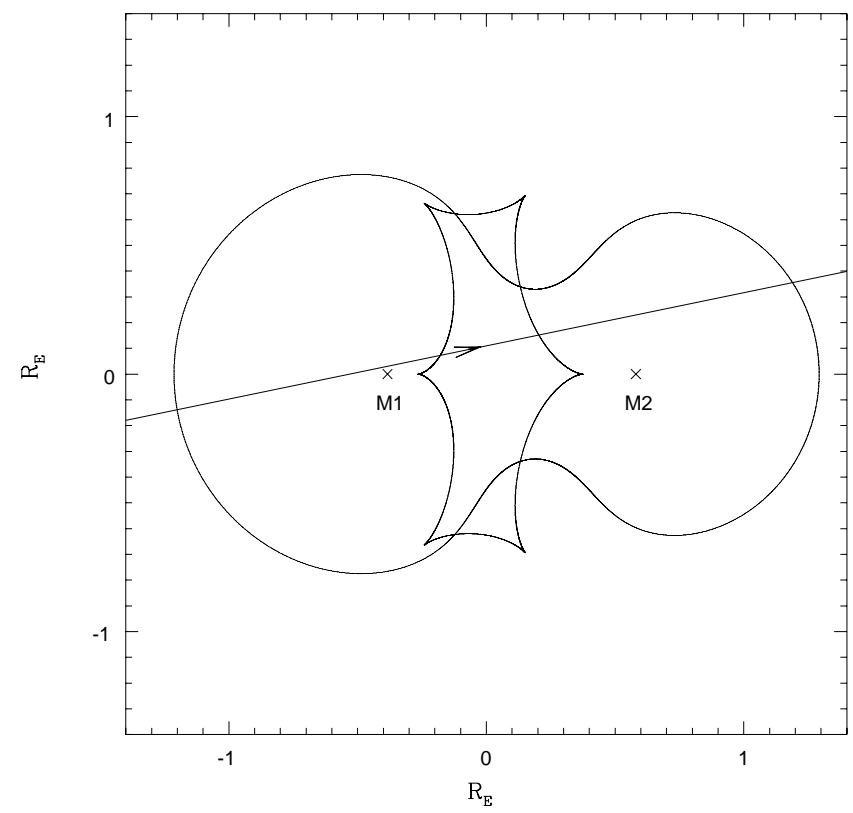

FIG. 44.- - Location of the (cuspy) caustic and (smooth) critical curves for the 176-A binary lens fit presented in Fig. 43. The coordinate system, whose origin is at the center of mass, indicates distance in units of the system's Einstein Ring radius $R_{\mathrm{E}}$. Also shown are the locations of the lensing objects, and the trajectory of the source through the caustic structure.

This MACHO object appears to be a clump giant, relatively unreddened compared with the other events in our sample. There is little blending in this event, such that the giant is likely the lensed source. The second caustic crossing in this model is resolved with two MACHO observations, which leads to an estimate of $t_{*}=0.53 \pm 0.03$ days. However, the lack of information prior to the start of the bulge season severely limits our ability to parameterize this event, so we expect that our fit may not be unique. The only strong constraints on this lensing encounter are the magnitude of the final cusp approach and the likelihood of minimal blending for a clump giant source.

\subsection{7-BLG-24 (101.20650.1216)}

The MACHO light curve for 97-BLG-24 (Fig. 24) exhibits a significant deviation from point source microlensing, similar to LMC-1 (Fig. 1). However, this deviation was noticed in real time, allowing immediate follow-up observations to be undertaken with the MSO 30 inch telescope. These data reveal an unusual increase in the object's brightness before observations ended for the evening.

Unfortunately, the lensed source appears $\sim 1 " 2$ from the MACHO object, in a region of high crowding. It is difficult to independently photometer the lensed source even in the follow-up photometry, and the data presented here represent the change in brightness of the brightest neighbor to the lensed source; i.e., the event is strongly blended. Nevertheless, we are able to resolve evidence of lens binarity in the deviation near the peak of the light curve. This deviation is more heavily sampled than LMC-1, but still suffers from the same model degeneracy. (We note that Fig. 24 displays the apparent brightening of the MACHO object, not the lensed source, because of the different blend fractions between the two fits presented in Tables 2 and 3.)

The solid and dashed line fits in Figure 24 display $\hat{t}$ of 30.7 and 45.5 days, implying overall lens masses of 0.19 and
$0.42 M_{\odot}$, respectively. The former fit includes a binary system with components of 0.16 and $0.03 M_{\odot}$, likely a starbrown dwarf system. The latter dashed fit, with a mass ratio of 29:1, implies lens masses of 0.41 and $0.014 M_{\odot}$, consistent with a stellar lens with a companion of $\sim 14$ Jupiter masses.

\subsection{7-BLG-28 (108.18951.593)}

After being detected and alerted upon 1997 May 29, this event began to increase in brightness at an unexpected rate on 1997 June 14 (day 1990 in Fig. 27), and both MACHO/ GMAN and PLANET issued secondary alerts for a binary lensing event in progress. The PLANET collaboration was able to obtain nearly constant coverage of this event, resulting in parameterization of the limb-darkening coefficients for the source and an estimate of the lens proper motion of $\mu=19.4 \pm 2.6 \mathrm{~km} \mathrm{~s}^{-1} \mathrm{kpc}^{-1}$ (Albrow et al. 1998b).

The results presented here are similar to those of Albrow et al. (1998b). The fit to microlensing suggests a moderate amount of unlensed blue light in the photometered object, and the object is likely a lensed clump giant source blended with objects of bluer color. The trajectory of the source plotted in Figure 28 indicates the light curve deviation was due to a cusp crossing. Resolution of the source face during this crossing allows a measurement of $t_{*}=0.760 \pm 0.014$ days. A comparison of event parameters (MACHO:PLANET) with model LD1 of Albrow et al. (1998b) yields $\hat{t}=(52.8: 54.4), \quad u_{\min }=(0.225: 0.215)$, $a=(0.71: 0.69), \epsilon_{1}=(0.17: 0.19), \theta=(1.44: 1.42)$, and $t_{*}=$ (0.76:0.78).

Following the procedure outlined in $\S$ 5.2.2 and Table 4, we estimate the reddening to be $E(V-R)=0.67 \pm 0.04$, which yields dereddened source magnitudes of $V=15.52 \pm 0.19$ and $R=14.94 \pm 0.15$. From this we determine $\bar{T}_{\text {eff }}=4500 \pm 200 \mathrm{~K}$ for the source star. Using a bolometric correction of $\mathrm{BC}_{V}=-0.48$, we find an angular source radius of $\theta_{*}=6.58 \pm 0.90 \mu$ as. For comparison, the PLANET group find a dereddened $V=15.27, T_{\text {eff }}=4350$ $\mathrm{K}$, and $\theta_{*}=8.74 \pm 1.17 \mu \mathrm{as}$, so our results are in reasonably good agreement.

\subsection{4. $97-B L G-41$ (402.47862.1576)}

This event was detected and alerted upon on 1997 June 18. This event exhibited what appeared to be a fairly normal rise and fall for a microlensing event. However, after peak the event did not decline to baseline magnification, but leveled off at $\mathrm{A} \sim 1.5$, and began a slow rise, which itself was fit well by a longer duration microlensing event. The deviation from a normal single lens light curve was noted and announced by both the MACHO/GMAN and PLANET collaborations. Near the peak of the event is an apparent caustic or cusp crossing. The MACHO and GMAN data have been plotted in Figure 29.

Considerable effort has been made to fit this light curve to a binary lens model, but no satisfactory model has been found, even when the possible orbital motion of the lens was included. However, a satisfactory multiple lens fit has been found by Bennett et al. (1999).

\subsection{8-BLG-12 (179.21577.1740)}

98-BLG-12 was detected on 1998 April 8, and initially thought to be a rapidly rising, high magnification event. This behavior, evident in Figure 30, was a result of the 

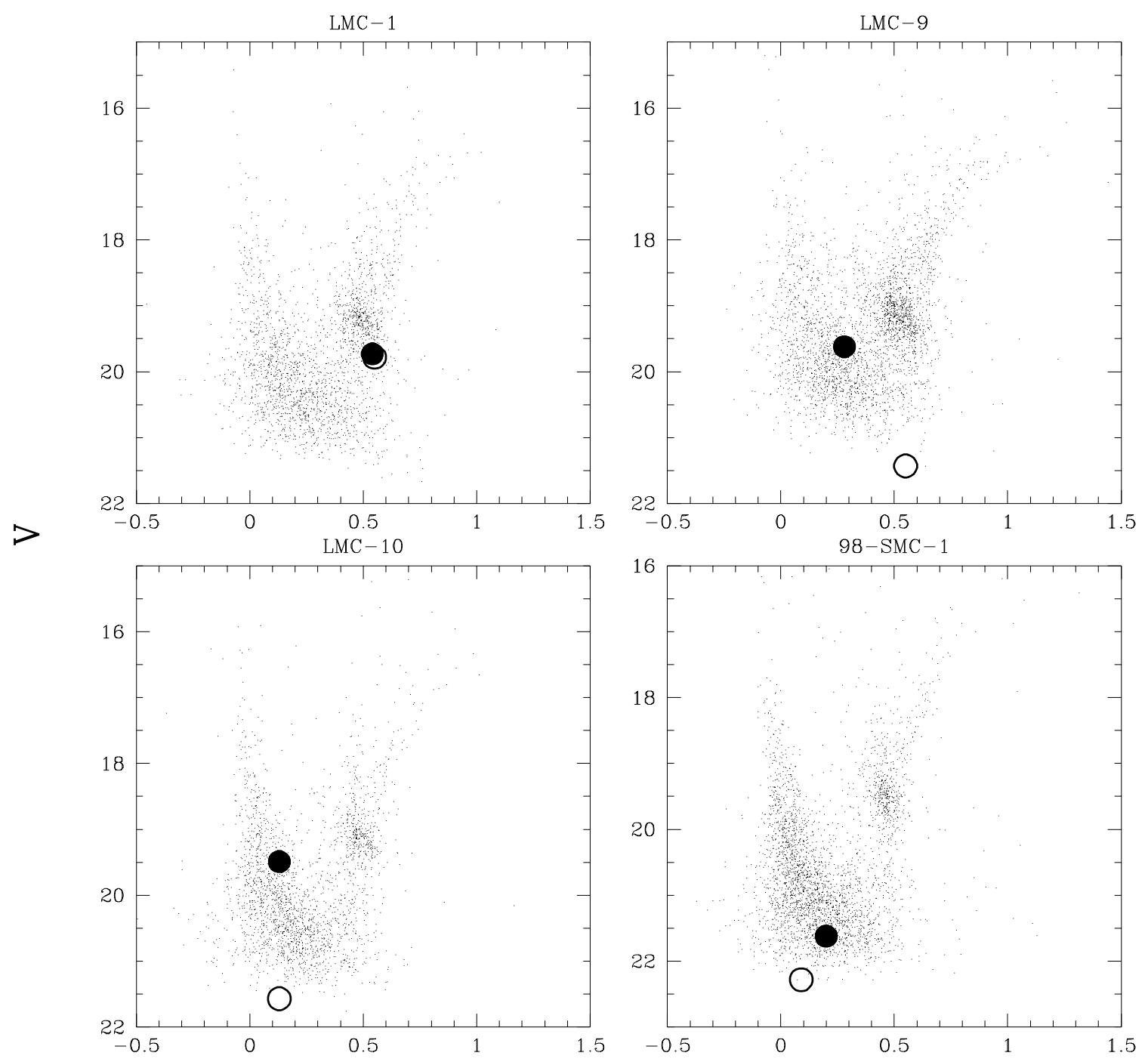

$\mathrm{V}-\mathrm{R}$

Fig. 45.-Distribution of $V, V-R$ for $\sim 3000$ stars neighboring the lensed object ( filled circle) for objects in the Magellanic Clouds. The deblended source location (open circle) is determined from blending parameters in the microlensing fit.

source exiting its first passage through the caustic structure (Fig. 31). It was not recognized as a binary lensing event until the source reentered the caustic structure near 1998 May 17.5 UT (day 2327.5) and was subsequently observed at high magnification by the survey telescope on 1998 May 17.74 UT (day 2327.74). The less than three days spent between caustics allowed little time for follow-up observations to constrain event parameters. Interestingly, in all passbands, this event appears heavily blended $(f \sim 0.2)$.

\subsection{6. $98-B L G-14(401.48408 .649)$}

This brightening of this apparent clump giant object was detected and alerted on 1998 April 26. Initially, it was not clear if the asymmetry in Figure 32 was due to the parallax effect (Refsdal 1966; Alcock et al. 1995), and initial data allowed fits of similar significance for both binary and parallax models. However, the higher precision photometry from CTIO and MSO74 observations clearly favor the binary interpretation over the best-fit parallax light curve $\left(\Delta \chi^{2}=115.75\right.$ with 1 less degree of freedom), while the
MACHO data also provide $\Delta \chi^{2}=30.32$. Unfortunately, there does remain a degeneracy between binary lens models. Figures 33 and 34 indicate this is a noncaustic crossing binary event, similar to 95-BLG-12.

The blend fraction for the best fit (solid lines in Figs. 32 and 33) is $f \sim 0.5$ for all four passbands of coverage, but there is another fit that is almost as good with $f \gtrsim 1$ (dashed lines in Figs. 32 and 34). The best fit suggests a blend of clump giant stars, where they are constrained to lie within a seeing disk, or $\sim 1^{\prime \prime}$. 98-BLG-14 is located closer to the Galactic center than most of our events at $l=1.96, b=$ -2.29 , where the surface density of giants is quite high. The average separation of clump giants is 5."3 in the vicinity of 98-BLG-14, so we expect that $\sim 10 \%$ of clump giants will be blended with another clump giant. The dereddened brightness of the MACHO object would place it at the bright tip of the clump. In the best fit, the lensed source would be somewhat lower than the mean clump brightness. It is thus reasonable for the lensed clump giant source to be blended with an unlensed clump giant. The second-best fit differs from the first by $\Delta \chi^{2}=5$, and from the event time- 


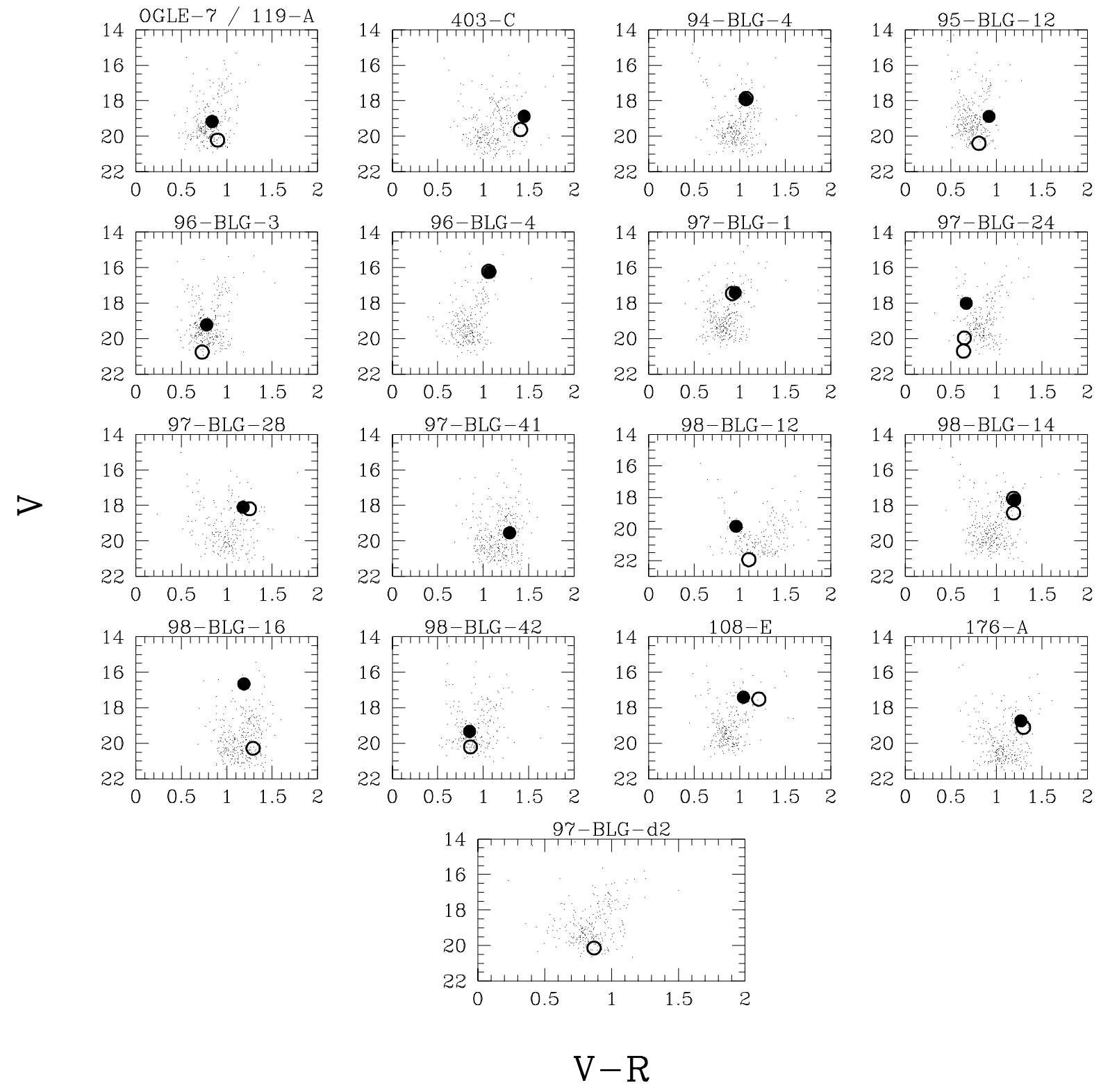

FIG. 46.-Distribution of $V, V-R$ for $\sim 3000$ stars neighboring the lensed object ( filled circle) for objects in the Galactic bulge. The deblended source location (open circle) is determined from blending parameters in the microlensing fit. Notable cases: $97-B L G-24$, for which the brighter source is associated with the low mass ratio fit in Fig. 24; 97-BLG-41, for which we have no estimate of the source properties; 98-BLG-14, for which the brighter source is associated with the large mass ratio fit in Fig. 32; 97-BLG-d2, for which we have only an estimate of the lensed source properties.

scale of $\hat{t}=74$ days and lens mass ratio of $12: 1$, we can estimate a lens system comprised of a $1.03 M_{\odot}$ primary and $0.088 M_{\odot}$ companion.

\subsection{7. $98-B L G-16(402.47863 .110)$}

98-BLG-16 was detected and alerted on 1998 April 28, and thought to be a high magnification, short timescale event. The initial sharp rise in Figure 35 was due to a cusp approach (Fig. 36). Subsequent light curve interpretation was hindered by a significant amount of scatter in the MACHO Red passband, because of a defective amplifier, and a centroid offset of $\sim 1$ 1"3 between the MACHO object and the lensed source. Follow-up data from the CTIO $0.9 \mathrm{~m}$ were able to resolve these objects, which allowed them to be independently photometered. Inspection of the CTIO data set indicates the lensed source has a baseline flux of $\sim 7 \%$ of the brightest (constant) neighboring star that serves as the target of MACHO photometry. The binary lens fit determines blend fractions consistent with this, $f_{\text {MACHO }} \sim 0.04$ and $f_{\text {CTIO }} \sim 1$, indicating there is no significant flux contribution from the lensing objects. The CTIO data from 1998 May 4.3 UT (day 2314.3) provide a constraint on the lens proper motion for this particular fit, $t_{*}=0.163 \pm 0.003$ days.

\subsection{8-BLG-42 (101.21045.2528)}

98-BLG-42 was detected and alerted on 1998 August 22 at a magnification of $\sim 4.0$. The source at this point in time was inside the caustic region depicted in Figure 38. The event was immediately followed up by the MPS effort, on the MSO 74 inch telescope. Over the next three nights the source began to rapidly increase in brightness. The PLANET collaboration issued an Anomaly Alert on 1998 August 26.0 UT (day 2428.0) indicating the source under- 
went a caustic crossing between 1998 August 25.0 and 25.7 UT. Our fit presented here indicates a caustic crossing date of August 25.77 UT, with a source radius crossing time of $t_{*}=0.109 \pm 0.016$ days.

\subsection{9. $97-B L G-d 2(108.19073 .2291)$}

This event was detected in the $3 \mathrm{yr}$ difference image analysis (DIA) of MACHO field 108, originally presented in Alcock et al. (1999b). The event is most closely associated with MACHO object 108.19073.2291, however the DIA technique uniquely identifies the time-varying source. The light curve associated with this analysis is purely a light curve of residuals around the source's baseline flux, which is not determined. For consistency with the notation of the other fits in this paper, we have added an arbitrary amount of flux to the residual light curve, and fit for the fraction of the combined light curve that is lensed. In this way we are able to estimate the baseline brightness of the lensed source. The source trajectory in Figure 40 includes two cusp approaches and passage through a caustic structure, which is suggested by two MACHO data points at high magnification.

\subsection{0. МАCHO-108-E (108.19333.1878)}

This event was detected in the course of the MACHO bulge $5 \mathrm{yr}$ analysis (Alcock et al. 2000, in preparation). The deviation occurred with the Alert System in place. However, the Alert System was not triggered since all but three of the MACHO Blue data points are removed from the light curve because of the object's proximity to the edge of a detector. For this reason we are unable to realistically estimate properties of the lensed source, or set meaningful limits on the lens brightness. The light curve (Fig. 41) is characterized by approaches to two of the three caustics in the source plane (Fig. 42).

\subsection{1. $\mathrm{MACHO-176-A} \mathrm{(176.19219.978)}$}

Event 176-A was also detected in the bulge 5 yr analysis and is a good example of a poorly sampled binary lens event. The magnitude of the initial caustic approach is unconstrained, and the fit presented here places the one relevant data point at its peak (Fig. 43). The $U$ shape of the subsequent data suggests a caustic crossing and gradual decline to baseline. The critical and caustic curves are presented in Figure 44.

\section{WHAT CAN WE LEARN?}

With our ensemble of 21 binary microlensing candidates, we can begin to consider mapping the properties of the binary events to the lensing population as a whole (Di Stefano 1999; Kerins \& Evans 1999). This is most difficult toward the Magellanic Clouds, where we have the additional uncertainty of an unknown or unmodeled lensing population.

\subsection{Toward the Magellanic Clouds}

We have presented three candidate LMC binary events out of the eight events published in Alcock et al. (1997b) and detect no more unambiguous binary lens candidates in the $\sim 20$ events (Alcock et al. 2000) in our 5 yr analysis (we do however detect one binary source candidate, 96-LMC-2, Becker et al. 1997). A color-magnitude diagram, incorporating the deblended magnitudes of the Magellanic sources from Table 1, can be found in Figure 45.
LMC-9 is a resolved caustic crossing event, where the measured $t_{*}$ (assuming a single lensed source) is consistent with the lensing system residing in the LMC (Bennett et al. 1996b), but only if the velocity dispersion and the selflensing optical depth (Gould 1995) of the LMC are both small. If the LMC self-lensing optical depth is large enough to explain most of the microlensing events seen toward the LMC (Sahu 1994), as in the recent models of Aubourg et al. (1999), then the proper motion of LMC-9 is not consistent with an LMC lens, unless the source star is actually a pair of binary stars of similar brightness. However, in this case, we can no longer constrain the proper motion of the lens.

As emphasized by Di Stefano (1999), we should also expect events without obvious caustic crossings, similar to LMC-10. This event is consistent with a binary lens event, but the asymmetry of the light curve also resembles what might be expected for some types of stellar variability. A future HST frame of this object could confirm the microlensing prediction for the amount of blending.

Event 98-SMC-1 was recognized to be a caustic crossing event in real time with the GMAN follow-up observations presented here. An unprecedented response by the majority of the microlensing community resulted in dense coverage of the event, including resolution of the second caustic crossing by the PLANET and EROS collaborations. Important constraints on the initial caustic crossing date were provided by the OGLE and MPS collaborations. The lens proper motion derived from each of these data sets is most consistent with a SMC lens. This is the strongest constraint that has yet been placed on the location of the lensing population toward the Magellanic Clouds. However, as Palanque-Delabrouille et al. (1998) point out, the SMC is expected to have a large self-lensing optical depth, so that a large fraction of SMC events are likely to be due to self-lensing, even if most of the LMC events are due to halo lenses.

Kerins \& Evans (1999) reach the conclusion that, if the caustic crossing events LMC-9 and 98-SMC-1 are both due to Magellanic lenses, than the bulk of lensing seen so far toward the Magellanic Clouds is most likely due to selflensing, where the lenses may reside in Magellanic stellar or dark halos. However, the suggestive evidence that the LMC-9 lens may reside in the LMC applies only for LMC models with a low self-lensing optical depth. Furthermore, as Honma (1999) has pointed out, there is probably a bias in favor of detecting long timescale caustic crossing events. Thus, we may be more likely to detect and characterize caustic crossing features for self-lensing events than for halo lensing events, if the latter tend to have shorter timescales. A potentially more serious bias may be that there may be very few binary lenses in the halo. If most of the LMC events are due to lenses in the Galactic halo, then they comprise a previously unknown population with an unknown binary fraction. So, it is possible that the sample of binary events themselves selects against the halo lensing events. If so, we might expect a smaller fraction of binary lensing events toward the LMC than toward the bulge when the event samples get larger.

\subsection{Toward the Galactic Bulge}

A more representative sample of binary lenses can be found among the 17 Galactic bulge candidates. For the duration 1994-1998, the Alert System triggered on 196 Galactic bulge microlensing events. Twelve of the Alert 
events are presented here as binary lens candidates. Since the Alert System is tuned to detect generic variability, it is reasonable to make the assumption that the 12 binary events out of the 196 Alert events are representative of the detectable binary fraction of the lensing population as a whole. This is consistent with theoretical estimates of a binary lensing rate of order 10\% (Mao \& Paczyński 1991).

A color-magnitude diagram, incorporating the deblended magnitudes of the lensed sources from Table 1, can be found in Figure 46. It is interesting to note that clump giants are overrepresented in our sample, implying our binary lens detection efficiency is highest with these bright sources. It is also important to note that, for the six clump giants lensed, in most cases the blending fraction is quite close to 1 (with the possible exception of 98-BLG-14), indicating there is insignificant contamination from neighboring sources and, most importantly, from the lensing system. This is contrary to the distributions of blending in the majority of binary microlensing events that have been reported in the literature thus far (Di Stefano 1999).

Two of the binary events (97-BLG-24 and 98-BLG-16) are heavily blended in the MACHO data, but the lensed source star is resolved in the follow-up GMAN data, at separations of 1.2 and 1 .'3, respectively. However, the 97-BLG-24 field is crowded enough that it is difficult to independently photometer the MACHO object and lensed source. For 98-BLG-16, the microlensing fit to CTIO data exhibits no blending, indicating the lens is in fact relatively dark. Candidates 95-BLG-12, 96-BLG-3, and 98-BLG-12 are strongly blended in all passbands $(70 \%-80 \%$ contamination), and it is possible in these cases that the lens contributes significant flux to the source brightness. Highresolution photometry of strongly blended events can disentangle blending due to crowding, and blending due to lens luminosity.

Excluding event 97-BLG-24 for which we have two fits, we find 12 (nine) caustic crossing events from our ensemble of 16 (only the 11 Alert) bulge candidates. While not all of the GMAN follow-up data for the interval 1995-1998 have yet been reduced, we so far have found no weakly perturbed systems, at the $\sim 1 \%$ level (see, however, Becker 2000, in preparation). Di Stefano (1999) indicates that several of these weakly perturbed events should exist for each caustic crossing event (but see $\S 2.1$ ). That we have detected no such events indicates our efficiency at detecting them, or characterizing them as binary microlensing, is currently quite low.

\subsubsection{Mass Ratio Distribution}

While some of the events here are poorly constrained, and thus can be characterized by multiple combinations of event parameters, we can begin to probe the distribution of mass ratios of the binary lensing population toward the Galactic bulge, given the above fits. Distributions of the binary parameters $\theta$ and $a$ are less informative, with $\theta$ representing a random orientation between the lens separation axis and motion with respect to the source, and $a$ the projection of the lens separation at a random (unknown) orbital phase. Figure 47 contains a histogram of the distribution of mass ratios ( $q$, defined to be $\leq 1)$ that we find from our bulge events. The two fits each for events 97-BLG24 and 98-BLG-14 are represented by additional shaded areas. The distribution here is free of inclination uncertainties present in studies of spectroscopic binaries (SB) but does likely suffer from nonuniqueness of fits in several cases.

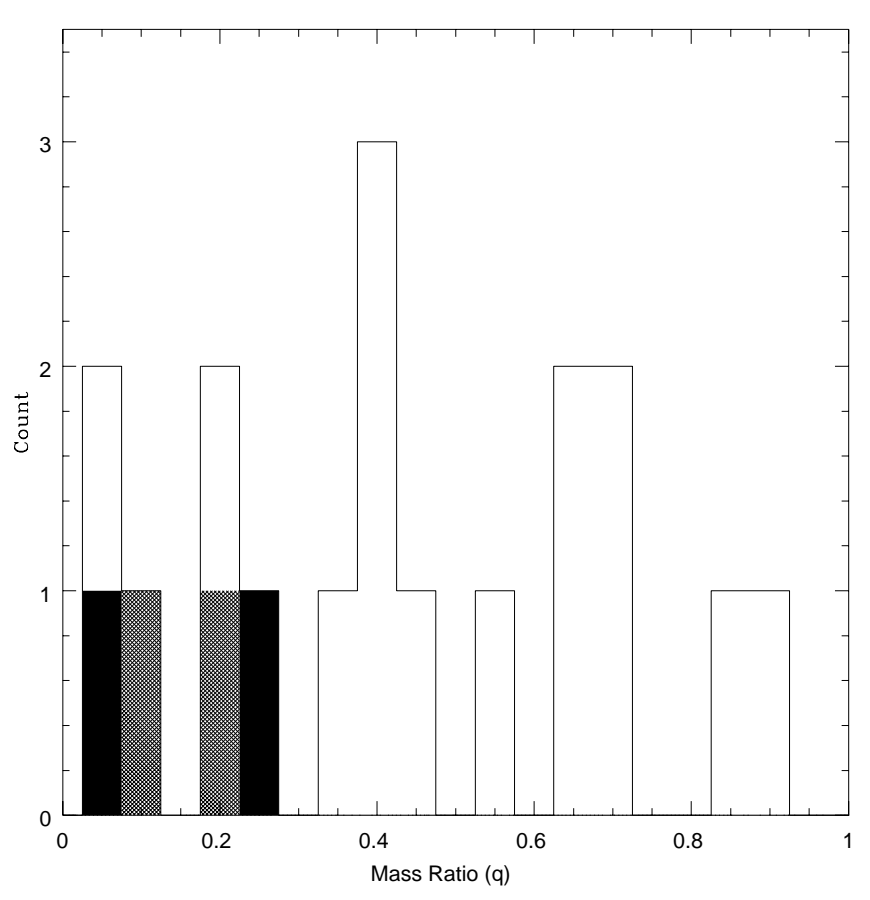

FIG. 47.-Distribution of mass ratios $(q \leq 1)$ for our Galactic bulge binary microlensing events. The contribution of our two fits each to events 97-BLG-24 and 98-BLG-14 are represented by the dark and light shaded areas, respectively.

The binary mass ratio distribution function $N(q)$ has been studied by, e.g., Trimble (1990), who examines SB systems with both giant and relatively bright main sequence primaries. Similar morphologies are found between samples, generally characterized by $N(q) \propto q^{-1}$, with a possible peak near $q \sim 0.3$. However, we expect many of our lenses to be drawn from the lower, more populated portion of the main sequence. Given the apparent dependence of $N(q)$ on spectral type (Abt 1983), it would be more appropriate to compare Figure 47 with the study of solar-type SB by Mazeh et al. (1992). They find, with a considerably smaller sample than Trimble (1990), a relatively flat $N(q)$, possibly rising toward larger $q$. We therefore compare two models, $N(q) \propto q^{-1}$ and $N(q)=$ constant, against the events with $0.1<q<1$ using the one-sided Kolmogorov-Smirnov (KS) test. We find probabilities of 0.07 and 0.41 , respectively, of finding a K-S deviation between data and model as large as that observed. $N(q) \propto q^{-1}$ is clearly inconsistent with our measured distribution, while a flat $N(q)$ is consistent with our data.

\subsubsection{Reddening Estimates}

For 10 of our bulge events, the microlensing fits provide a measurement of $t_{*}$. However, it is our coverage of events 119-A (Fig. 10), 96-BLG-3 (Fig. 18), 97-BLG-28 (Fig. 27), and 98-BLG-42 (Fig. 37) that most reliably constrains $t_{*}$.

We therefore have four events where we believe we have a reliable measurement of the time it takes the lens to transit the source face, $2 t_{*}$. To arrive at an estimate of the proper motion of the lensing system, we must first determine the angular size of the source. This can be done by assuming the source is a blackbody and estimating its effective temperature $T_{\text {eff }}$ and apparent bolometric magnitude $m_{\text {bol }}$. We first need to determine the extinction, $A_{V}$, and reddening, $E(V-R)$, to the source - in the following, we assume $A_{V}=$ 3.97E(V-R) (Rieke \& Lebofsky 1985). 
TABLE 4

INTRINSIC SOURCE PROPERTIES

\begin{tabular}{ccccccccc}
\hline \hline Event & Number of RR Lyrae & $\langle E(V-R)\rangle$ & $\left\langle A_{V}\right\rangle$ & $(V-R)_{0}$ & $V_{0}$ & $\begin{array}{c}T_{\text {eff }} \\
(\mathrm{K})\end{array}$ & $\begin{array}{c}\theta_{*} \\
(\mu \text { as })\end{array}$ \\
\hline $119-A \ldots \ldots \ldots \ldots$ & 3 & $0.47(9)$ & $1.9(3)$ & $0.43(9)$ & $18.4(3)$ & $5350(500)$ & $18.2(4)$ & $1.06(29)$ \\
96-BLG-3 ....... & 6 & $0.42(5)$ & $1.7(2)$ & $0.31(6)$ & $19.1(2)$ & $6200(500)$ & $19.1(2)$ & $0.53(11)$ \\
$97-B L G-28 \ldots \ldots$ & 9 & $0.67(4)$ & $2.7(2)$ & $0.58(6)$ & $15.5(2)$ & $4500(200)$ & $15.0(2)$ & $6.58(90)$ \\
$98-B L G-42 \ldots \ldots$ & 10 & $0.53(5)$ & $2.1(2)$ & $0.33(7)$ & $18.1(2)$ & $6050(500)$ & $18.1(2)$ & $0.89(20)$ \\
\hline
\end{tabular}

Notes.-Estimates of the reddening and extinction, as determined from RR Lyrae within $10^{\prime}$, to each of the four sources for which we have a well-constrained binary lens fit and a reliable measure of $t_{*}$. The intrinsic source color $(V-R)_{0}$ and brightness $V_{0}$ are used to determine $T_{\text {eff }}$ and $m_{\mathrm{bol}}$, and from these we find the source angular radius $\theta_{*}$.

Stanek (1996), in a study of red clump stars in Baade's Window, finds a range of $A_{V}$ from 1.26 to 2.79 , even though this is the clearest and most uniform window through the bulge. This suggests it would be unwise to apply an "average" extinction correction to all of our events. Instead, we estimate the reddening for each source using neighboring RR Lyrae stars, whose intrinsic colors are well known. An intrinsic color-period $(P)$ relationship has been established for field RR Lyrae by Caputo \& De Santis

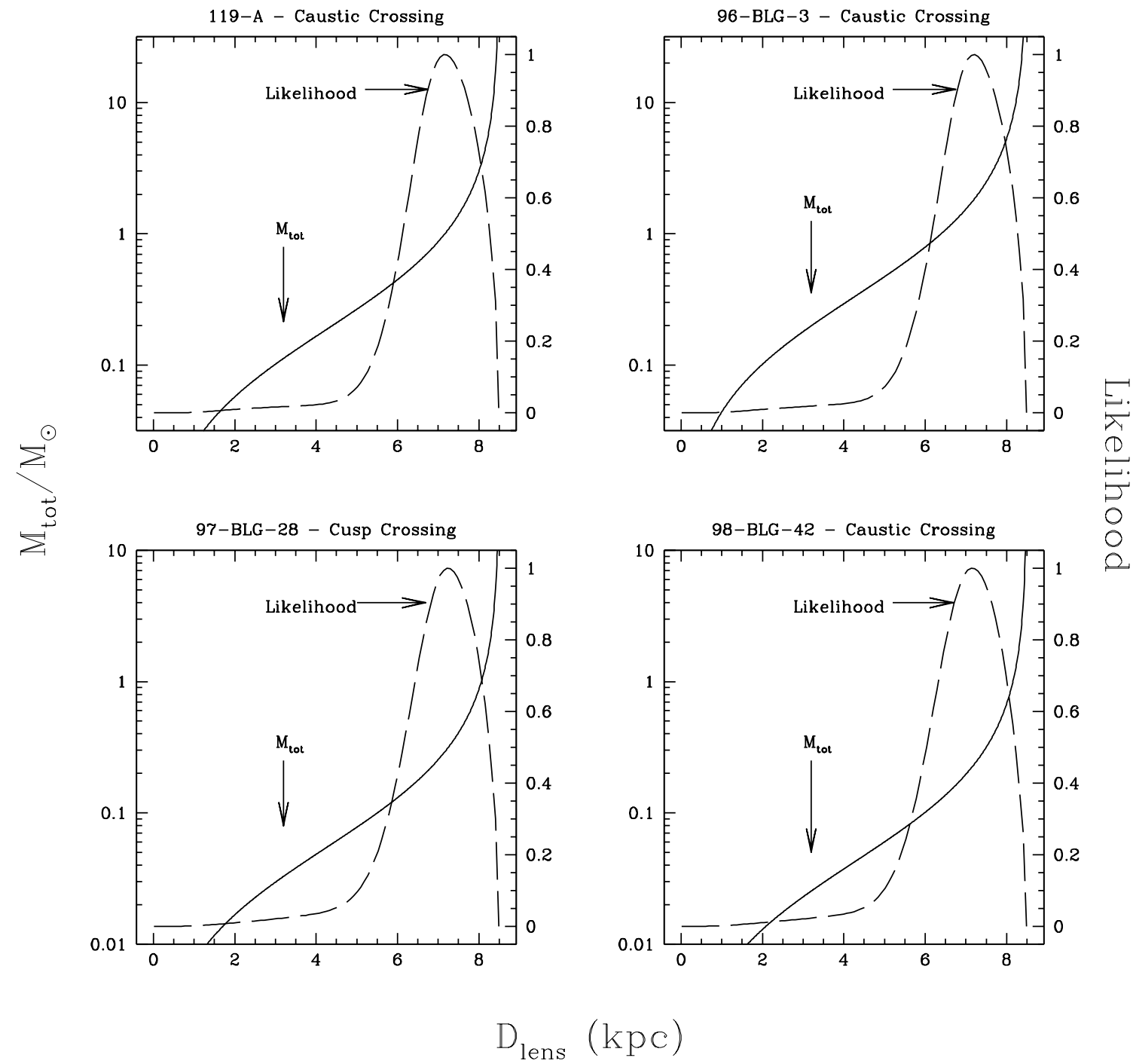

(1992), in the form

$$
(B-V)_{0}=0.658+0.097[\mathrm{Fe} / \mathrm{H}]+0.710 \log P .
$$

We assume $[\mathrm{Fe} / \mathrm{H}]=-1$, after Walker $\&$ Terndrup (1991), and transform to $(V-R)_{0}$ with the relation (Alcock et al. 1997a)

$$
(V-R)_{0}=0.004+0.566(B-V)_{0}
$$


TABLE 5

Properties of The Lensing Systems

\begin{tabular}{|c|c|c|c|c|c|}
\hline Event & $\begin{array}{c}\mu \\
\left(\operatorname{mas~} \mathrm{yr}^{-1}\right)\end{array}$ & $\begin{array}{c}D_{o l} \\
(\mathrm{kpc})\end{array}$ & $M_{\text {tot }} / M_{\odot}$ & $M_{1} / M_{\odot}$ & $M_{2} / M_{\odot}$ \\
\hline 119-A. & $1.84(58)$ & $7.0_{-0.9}^{+0.8}$ & $0.89_{-0.42}^{+1.25}$ & $0.49_{-0.23}^{+0.69}$ & $0.40_{-0.19}^{+0.57}$ \\
\hline 96-BLG-3 ....... & $2.26(47)$ & $7.1_{-0.9}^{+0.8}$ & $1.61_{-0.76}^{+2.25}$ & $0.45_{-0.21}^{+0.63}$ & $1.15_{-0.55}^{+1.62}$ \\
\hline 97-BLG-28 ...... & $3.16(44)$ & $7.0_{-1.0}^{+0.8}$ & $0.26_{-0.13}^{+0.36}$ & $0.05_{-0.02}^{+0.06}$ & $0.22_{-0.11}^{+0.30}$ \\
\hline 98-BLG-42 ...... & $2.98(78)$ & $7.0_{-1.0}^{+0.8}$ & $0.19_{-0.09}^{+0.26}$ & $0.14_{-0.07}^{+0.20}$ & $0.05_{-0.02}^{+0.06}$ \\
\hline
\end{tabular}

Notes.-The lens proper motion measurements, $\mu=\theta_{*} / t_{*}$, have been used in the likelihood analysis detailed in $\S 5.2 .3$ to produce the most likely lens distance and mass estimates given here. The errors quoted are $1 \sigma$. We note that the errors in $M_{1}$ and $M_{2}$ are completely correlated.

We then compute the reddening to all RR Lyrae within $10^{\prime}$ of the source, excluding significant outliers, and apply the reddening and extinction corrections to our lensed source star. The results of this are listed in the first columns of Table 4. We note that two of the events, 119-A and 96-BLG-3, occurred within Baade's Window, but both sources are just outside the extinction grid reported by Stanek (1996).

We next interpolate the ATLAS9 and NMARCS model atmospheres presented in Bessell, Castelli, \& Plez (1998; assuming solar metallicity, $\log g=2.0$ for giants, and $\log g=4.5$ otherwise) to determine the source's $T_{\text {eff }}$ and bolometric correction in $\mathrm{V}\left(\mathrm{BC}_{V}\right)$, given its unreddened $(V-R)_{0}$. It is then straightforward to calculate the angular size of the source $\theta_{*}$ using the bolometric flux method of Gray (1992), and to determine the relative proper motion $\mu=\theta_{*} / t_{*}$ between the lens and the source, further described in $\S 5.2 .3$.

\subsubsection{Lens Proper Motions}

The determination of the lens proper motion with respect to the source, $\mu$, yields an additional constraint on the parameters of the lensing system. For most lensing events, the only observable parameter that constrains the distance, $D_{o l}$, total mass, $M_{\mathrm{tot}}$, and the transverse velocity, $v_{\perp}$, of the lens system is the event timescale, $\hat{t}$. However, for caustic crossing events, we have seen that it is also possible to measure the lens proper motion, which is related to the distance and transverse velocity of the lens by

$$
\mu=\frac{v_{\perp}}{D_{o l}} .
$$

Thus, for the four events with measured $\mu$ values, we have two constraints on three parameters, so there is a oneparameter family of solutions, namely,

$$
M_{\mathrm{tot}}=\frac{\mu^{2} \hat{t}^{2}}{16 G} \frac{D_{o l}\left(D_{o l}+D_{l s}\right)}{D_{l s}} .
$$

These solutions are shown in Figure 48. We see that $M_{\text {tot }}$ grows as function of the lens distance, and passes through the expected lens masses of $0.1-2 M_{\odot}$ at distances of 2-7.5 kpc.

We can make a more accurate estimate of the lens parameters if we make use of our knowledge of the velocity distributions of the source and lens populations. This requires that we specify a Galactic model, and we select the simple bar model of Han \& Gould (1995). The kinematics of the bulge using giants has been measured by Minniti (1996). We assume velocity dispersions of $30 \mathrm{~km} \mathrm{~s}^{-1}$ in each transverse direction for disk lenses, and $80 \mathrm{~km} \mathrm{~s}^{-1}$ in each transverse direction for both lenses and source stars located in the central bar. We have assumed a distance of $R_{0}=8 \mathrm{kpc}$ to the Galactic center and $8.5 \mathrm{kpc}$ to each source star. We have done a likelihood analysis to determine the most probable lens distance and mass for each of the four events with reliable proper motion measurements, and the results are summarized in Figure 48 and Table 5. The measured proper motions of $\mu=1.8-3.2$ mas $\mathrm{yr}^{-1}$ are most consistent with lens systems residing in the bulge, and the best-fit primary lens masses are consistent with main-sequence stars fainter than the Sun. The only exception is the heavier lens mass for event 96-BLG-3, which has a best-fit mass of $1.2 M_{\odot}$ with an uncertainty of a factor of two.

If the heavier 96-BLG-3 lens is a main-sequence star, then we must have $M_{2} \leq 1.3 M_{\odot}$ to be consistent with our measurement of the unlensed flux observed at the position of the source star. High resolution imaging of the source might be able to tighten this constraint by resolving some of the unlensed flux into separate stars. Similar constraints apply for the other stars but are significantly weaker because the implied masses are much lower. However, these constraints do not apply for stellar remnant lenses, which may make up of order $20 \%$ of the lens population (Gould 2000).

\section{CONCLUSIONS}

After a survey of the MACHO database, it is very apparent that microlensing by binary lens systems has been detected and at a rate that is roughly consistent with theoretical predictions for known stellar populations. However, a rigorous search has not yet been implemented, and we cannot set hard limits on the binary microlensing rate nor on the characteristics of binary systems in the lensing population. This includes the incidence of planets around lensing stars. However, follow-up efforts such as MPS (Rhie et al. 1998) and PLANET (Gaudi et al. 1998b) are undertaking dense light curve sampling and are beginning to set meaningful limits on planetary companions on an event-by-event basis.

It is also apparent that there are difficult degeneracies between binary microlensing fits that cannot be resolved with the sparse sampling (roughly once per night) of the microlensing survey telescopes. This is especially important in short duration and/or low level deviations, such as caustic crossings or planetary "spikes." In both cases important information is contained in a small fraction of the light curve. It is thus important that microlensing follow-up continues with dense (tens of observations per night), precise $(\sim 1 \%)$ sampling of event light curves. The 
limitations of undersampled data sets are apparent in the analysis of 97-BLG-24, where we are sure of a binary (possibly even planetary) signal but are not able to uniquely characterize it.

Three of our bulge events (95-BLG-12, 96-BLG-3 and 98-BLG-12) are strongly blended in all MACHO and GMAN follow-up passbands, indicating the lensing objects may be luminous at a detectable level. High-resolution observations of these sources may eventually reveal the appearance of a "new" source, as the lens proper motion separates it from the lensed source at the rate of $\sim$ mas $\mathrm{yr}^{-1}$. This can be accomplished with the HST, or adaptive optics imaging available on systems such as Gemini. On the other hand, our binary events on giant sources tend to show little blending. Event 98-BLG-16, a main-sequence source that is highly blended in the MACHO photometry, is resolved into separate objects in GMAN follow-up data, and fit blend fractions indicate the lensing objects are in fact dark.

We have recovered a distribution of mass ratios for 16 of our Galactic bulge microlensing candidates, and a distribution of lens proper motions for four of these events where we have, to some degree, resolved a caustic crossing. The mass ratio distribution is consistent with the relatively flat distribution seen in solar-type spectroscopic binary systems. The lens proper motions, when combined with the likelihood analysis in $\S 5.2 .3$, imply a population of binary lenses residing in the Galactic bulge at a distance of $7 \pm 1 \mathrm{kpc}$. The lens masses generally appear subsolar.

Finally, we would like to caution against overinterpretation of this data set, in particular the Magellanic Cloud subset, which is admittedly incomplete. In fact, there are undoubtedly a number of unquantified biases in our ability to discriminate between the single-lens and binary-lens case, although actual event detection is more a function of the significance of the deviation from baseline than light curve morphology. However, observations of ongoing binary lensing events toward the Magellanic Clouds, such as 98-SMC-1, may on an event-by-event basis allow us to examine the role the lensing populations play in the larger context of Galactic dark matter.

We are very grateful for the skilled support given our project by S. Chan, S. Sabine, and the technical staff at the Mt. Stromlo Observatory. We especially thank J. D. Reynolds for the network software that has made this effort successful. We would like to thank the many staff and observers at CTIO and UTSO who have helped to make the GMAN effort successful.

Work performed at LLNL is supported by the DOE under contract W7405-ENG-48. Work performed by the Center for Particle Astrophysics personnel is supported in part by the Office of Science and Technology Centers of NSF under cooperative agreement AST-8809616. Work performed at MSSSO is supported by the Bilateral Science and Technology Program of the Australian Department of Industry, Technology and Regional Development. Astronomy at Wise Observatory is supported by a grant from the Israel Science Foundation. K. G. acknowledges support from DOE grant DEFG03-90-ER 40546 and a Cottrell Scholar award. D. M. is also supported by Fondecyt 1990440. C. W. S. thanks the Packard Foundation for their generous support. W. J. S. is supported by a PPARC Advanced Fellowship.

\section{REFERENCES}

Abt, H. A. 1983, ARA\&A, 21, 343

Afonso, C., et al. 1998, A\&A, 337, L17 2000, ApJ, 532, 340

Alard, C., Mao, S., \& Guibert, J. 1995a, A\&A, 300, L17

Alard, C., et al. 1995b, ESO Mess., 80, 31

Albrow, M., et al. 1996, in IAU Symp. 173, Astrophysical Applications of Gravitational Lensing, ed. C. S. Kochanek \& J. N. Hewitt (Dordrecht: Kluwer), 227

. 1998a, ApJ, 509, 687

1998b, preprint (astro-ph/9811479)

1999a, ApJ, 512, 672

1999b, ApJ, 522, 1011 1999 c, ApJ, 522, 1022

Alcock, C., et al. 1993, Nature, 365, 621

1995, ApJ, 454, L125

1997a, ApJ, 482, 89

$1997 \mathrm{~b}$, ApJ, 486, 697

1997c, ApJ, 490, L59

1997d, ApJ, 491, 436

1998a, ApJ, 492, 190

1998b, ApJ, 494, 396

1998c, ApJ, 499, L9

1999a, ApJ, 518, 44

1999 b, ApJ, 521, 602

1999c, PASP, 111, 1539

2000, ApJ, submitted

Aubourg, E., et al. 1993, Nature, 365, 623

. 1999, A\&A, 347, 850

Becker, A. C., et al. 1997, BAAS, 191, 8305

1998, IAU Circ., 6935, 1

Bennett, D. P., \& Rhie, S. H. 1996, ApJ, 472, 660

Bennett, D. P., et al. 1993, BAAS, 183, 7206

1995, in AIP Conf. Proc. 336, Dark Matter, ed. S. S. Holt \& C. L.

Bennett (New York: AIP), 77

1996a, IAU Circ., 6361, 1

1996b, in Nucl. Phys. B Suppl., 51, 131

1997, in Proc. Planets Beyond the Solar System and the Next Generation of Space Missions, ed. D. R. Soderblom (San Francisco: ASP), 95
Bennett, D. P., et al. 1998, IAU Circ., 6939, 1

Bessell, M. S., Castelli, F., \& Plez, B. 1998, A\&A, 333, 231

Bolatto, A. D., \& Falco, E. E. 1994, ApJ, 436, 112

Caputo, F., \& De Santis, R. 1992, AJ, 104, 253

Cowley, A. P., \& Hartwick, F. D. A. 1991, ApJ, 373, 80

Di Stefano, R. 1999, preprint (astro-ph/9901035)

Di Stefano, R., \& Mao, S. 1996, ApJ, 457, 93

Di Stefano, R., \& Perna, R. 1997, ApJ, 488, 55

Di Stefano, R., \& Scalzo, R. A. 1999a, ApJ, 512, 564

. 1999b, ApJ, 512, 579

Dominik, M. 1998a, A\&A, 329, 361 1998b, A\&A, 330, 963 1999, A\&A, 341, 943

Dominik, M., \& Hirshfeld, A. C. 1996, A\&A, 313, 841

Gaudi, B. S., Naber, R. M., \& Sackett, P. D. 1998a, ApJ, 502, L33

Gaudi, B. S., et al. 1998b, BAAS, 193, 10807

Gould, A. 1995, ApJ, 441, 77

. 2000, ApJ, 535, 928

Gould, A., \& Loeb, A. 1992, ApJ, 396, 104

Gray, D. F. 1992, The Observation and Analysis of Stellar Photospheres

(2d ed.; Cambridge: Cambridge Univ. Press)

Griest, K., \& Safizadeh, N. 1998, ApJ, 500, 37

Han, C., \& Gould, A. 1995, ApJ, 447, 53

Hart, J., et al. 1996, PASP, 108, 220

Honma, M. 1999, ApJ, 511, L29

Iben, J., I. 1967, ARA\&A, 5, 571

Kerins, E., \& Evans, N. 1999, ApJ, 517, 734

Lennon, D. J., Mao, S., Fuhrmann, K., \& Gehren, T. 1996, ApJ, 471, L23

Mao, S., \& Di Stefano, R. 1995, ApJ, 440, 22

Mao, S., \& Paczyński, B. 1991, ApJ, 374, L37

Mao, S., et al. 1994, BAAS, 185, 1705

Marshall, S., et al. 1994, in IAU Symp. 161, Astronomy from Wide-Field Imaging, ed. H. T. MacGillivray et al. (Boston: Kluwer), 67

Mazeh, T., Goldberg, D., Duquennoy, A., \& Mayor, M. 1992, ApJ, 401, 265

Minniti, D. 1996, ApJ, 459, 579

Paczyński, B. 1986, ApJ, 304, 1

. 1991, ApJ, 371, L63

Paczyński, B., \& Stanek, K. Z. 1998, ApJ, 494, L219 
Palanque-Delabrouille, N. 1997, Ph.D. thesis, Univ. Paris 7

Palanque-Delabrouille, N., et al. 1998, A\&A, 332, 1

Peale, S. J. 1997, Icarus, 127, 269

Pratt, M., et al. 1996, in IAU Symp. 173, Astrophysical Applications of Gravitational Lensing, ed. C. S. Kochanek \& J. N. Hewitt (Dordrecht: Kluwer), 221

Refsdal, S. 1966, MNRAS, 134, 315

Renault, C., et al. 1997, A\&A, 324, L69

Rhie, S. H., \& Bennett, D. P. 1996, in Nucl. Phys. B Suppl., 51, 86

Rhie, S. H., Becker, A. C., Bennett, D. P., Fragile, P. C., Johnson, B. R., King, L. J., Peterson, B. A., \& Quinn, J. 1999, ApJ, 522, 1037

Rhie, S. H., et al. 1998, BAAS, 193, 10805

Rieke, G. H., \& Lebofsky, M. J. 1985, ApJ, 288, 618

Sahu, K. C. 1994, Nature, 370, 275

Schechter, P. L., Mateo, M., \& Saha, A. 1993, PASP, 105, 1342

Schneider, P., \& Weiss, A. 1986, A\&A, 164, 237
Stanek, K. Z. 1996, ApJ, 460, L37

Stanek, K. Z., Mateo, M., Udalski, A., Szymański, M., Kaluzny, J., \& Kubiak, M. 1994, ApJ, 429, L73

Stetson, P. 1987, PASP, 99, 191

Stetson, P. B. 1994, PASP, 106, 250

Tomaney, A. B., \& Crotts, A. P. S. 1996, AJ, 112, 2872

Trimble, V. 1990, MNRAS, 242, 79

Udalski, A., Kubiak, M., \& Szymański, M. 1997, Acta Astron., 47, 319

Udalski, A., et al. 1993, Acta Astron., 43, 289 . 1994, ApJ, 436, L103 1998, preprint (astro-ph/9808077)

Walker, A. R., \& Terndrup, D. M. 1991, ApJ, 378, 119

Zaritsky, D., Shectman, S. A., Thompson, I., Harris, J., \& Lin, D. N. C. 1999, AJ, 117, 2268

Zhao, H., Rich, R. M., \& Spergel, D. N. 1996, MNRAS, 282, 175 